\title{
Checklist and annotated bibliography of decapod crustacean larvae from the Southwestern European coast (Gibraltar Strait area)*
}

\author{
J.I. GONZÁLEZ-GORDILLO ${ }^{1,3}$, A. DOS SANTOS² and A. RODRÍGUEZ1 \\ ${ }^{1}$ Instituto de Ciencias Marinas de Andalucía (CSIC), Campus Universitario Río San Pedro, Apdo Oficial. \\ E-11510 Puerto Real, Cádiz, Spain. \\ ${ }^{2}$ Instituto de Investigação das Pescas e do Mar (IPIMAR), Avd de Brasilia, s/n. P-1449-006 Lisboa, Portugal. \\ ${ }^{3}$ Present address: Departamento de Biología, Universidade de Aveiro, 3810-193 Aveiro, Portugal. \\ E-mail: gonzalez@bio.ua.pt
}

\begin{abstract}
SUMMARY: Studies on decapod larvae entailing identification of specimens collected from plankton should be based on sound bibliographic sources that contain morphological descriptions of the species cited in the area. In order to facilitate this bibliographic search, we present a survey of the literature in which decapod larvae from the Gibraltar Strait area (encompassing most of the Mediterranean and northeastern Atlantic decapod crustacean species) are partially or completely described. Among these, the most accuratly identified larval specimens are pointed out. For each original case the taxon assigned by the author, types and numbers of stages described and the origin of the studied larvae are noted. Morever, a checklist of 377 species is given for Gibraltar Strait decapod crustaceans. Of all the species recorded in the area, larval descriptions are known, at least partially, for 220 species $(58,35 \%)$, but for $36(9,54 \%)$ morphological descriptions are sufficiently accurate for a correct identification from plankton specimens.
\end{abstract}

Key words: Decapoda, larvae, bibliography, checklist, Gibraltar Strait.

\section{INTRODUCTION}

The identification of decapod larvae from plankton samples proves to be a complex task, mainly because of the wide dispersion of bibliographic sources. Easy access to detailed descriptions, clear illustrations, and suitable keys are much help in the identification of specimens. The quality of descriptions and illustrations of many publications, however, is not always acceptable, making the compilation of complementary information from different reports essential.

Many of the early workers gave only brief descriptions of larvae, often without illustrations,

\footnotetext{
*Received April 28, 2000. Accepted September 29, 2000.
}

and only few identified the parental individuals to species level. In addition, many of the species described then are now ascribed to other species, genera, or even families. This results in a serious difficulty in the collection of all the existing bibliography on a given species, which may have been referred to under various names. To avoid this, the present work has taken into account such modifications, indicating for each reference the current name of the taxon and that ascribed (used) by the author of the description.

Since the first description of a decapod larva (Cancer germanus, now Cancer pagurus) by Linnaeus in 1767 , many larval developments have been published, especially in the second half of the 20th century. In an excellent review, Gurney (1939) gath- 
ered all the existing literature at the time. Since then, more specific reviews have been carried out for brachyuran larvae (Soltanpour-Gargari et al., 1989), the family Xanthidae (Martin, 1984), the New Zealand fauna (Wear, 1985), and the Mediterranean larvae (Pessani and Robotti, 1992).

The present contribution provides an updated database of all the available descriptions of larval stages of decapod crustaceans from the Gibraltar Strait area, so as to give bibliographic support for the identification and morphological comparison of specimens collected from the plankton and reared in the laboratory.

\section{MATERIAL AND METHODS}

To make up the list of the species present in the Gibraltar Strait all the species recorded for the area comprised between $30^{\circ}-40^{\circ} \mathrm{N}$ and $0^{\circ}-10^{\circ} \mathrm{W}$ have been included (Fig. 1). With this purpose, the papers of Nöel (1992), Ingle (1993), García Raso (1996) and d'Udekem d'Acoz (1999), as well as personal observations, have been used.

The search of bibliographic references has been mainly done through the following data bases: Current Contents, Zoological Records, Biological Abstracts and ASFA Aquaculture Abstracts, as well as bibliographic sources of several libraries up to year 2000. The references which have been chosen provide descriptive data facilitating the identification of the specimens, whereas references that include only data on larval presence or deal with ecological and physiological aspects have not been considered. Likewise, references pertaining to taxonomic categories above the species level have not been included if the material studied was from localities outside of the considered area (i.e., "Upogebia sp. A: Ngoc-Ho, N. 1981" from planktonic samples from the Gulf of México).

Each of the references should be interpreted as follows:

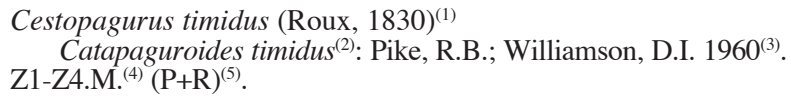

(1) currently accepted name of the taxon; (2) taxon to which the author(s) assign to the studied material; (3) author(s) and publication date of larval description; and (4) larval stages described. The original name of every larval stage described is retained to provide the largest amount of information, being A

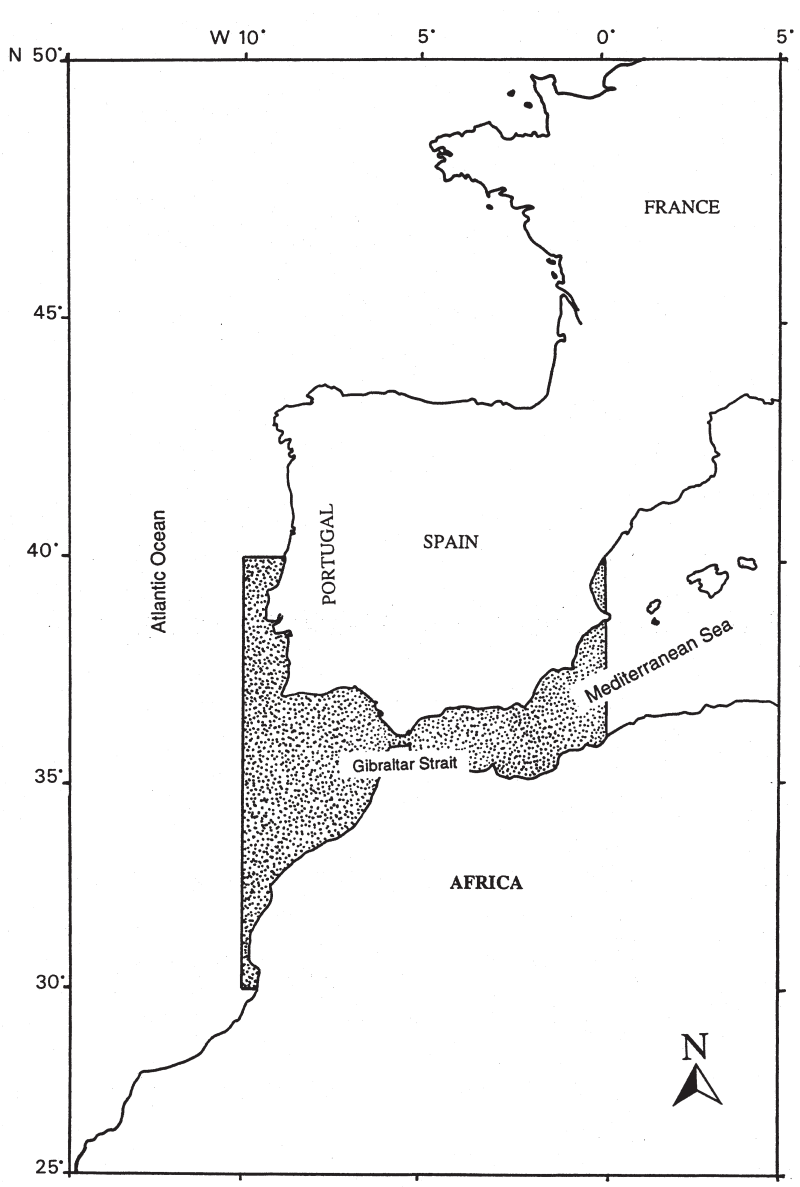

Fig. - 1. Map showing the location of the study zone (dotted area).

= acanthosoma, $\mathrm{C}=$ retrocaris, $\mathrm{D}=$ eretmocaris, $\mathrm{E}=$ elaphocaris, $\mathrm{F}=$ trachelifer, $\mathrm{G}=$ glaucothoe, $\mathrm{H}=$ anebocaris, $\mathrm{L}=$ nauplius, $\mathrm{LS}=$ last stage, $\mathrm{M}=$ megalopa, $\mathrm{N}=$ nisto, $\mathrm{P}=$ phyllosoma, $\mathrm{PL}=$ postlarva, $\mathrm{PS}$ = penultimate stage, $\mathrm{PT}=$ protozoea, $\mathrm{Q}=$ eryoneicus; $\mathrm{S}=$ Schizopod, $\mathrm{T}=$ mastigopus, $\mathrm{U}=$ puerulus, $\mathrm{Y}=$ mysis, and $\mathrm{Z}=$ zoea. $\mathrm{NE}$, indicates that larval stages are not specified hastly (5) refers to the origin of the description where $(\mathrm{L})=$ larvae reared in the laboratory from eggs of known parentage, $(\mathrm{P})=$ descriptions based on stages found in the plankton, (R) = larvae reared in the laboratory from larvae caught in field, from unknown parentage often to reconizable postlarvae or juveniles, and $(\mathrm{B})=$ after others publications.

Other symbols and abbreviations should be interpreted as follows: $\mathrm{K}$, means that mentioned taxon is included in an identification key; ?, indicates that the identity of taxon or stage is in question (by author or currently); *, indicates that the work, in our opinion, is sufficiently strong to establish species identify beyond reasonable doubt; NL, means that work has 
not been able to be consulted; and CG, means general features of larval stages are mentioned.

Although the postlarval stage of Dendrobranchiata and Caridea is not strictly a larval stage some researchers have considered it the last larval stage. In this study we have included this data (signed as PL) as additional information.

The systematic classification followed is that proposed by Bowman and Abele (1982) and Burkenroad (1983).

\section{RESULTS}

In the Gibraltar Strait (sensu lato) 377 species of decapod crustaceans have been recorded. For these species, an account of 387 scientific papers, in which larval descriptions are shown, have been selected up to the year 2000. At least one of the larval stages has been described for 220 species $(58,35$ $\%)$, and from only 36 species $(9,54 \%)$ accurate larval descriptions are available to allow a correct identification from plankton larvae.

Complete larval development is still not known for species belonging to the families Aristeidae, Benthysicymidae, Solenoceridae, Nematocarcinidae, Nephropidae, Axiidae, Calocarididae, Polychelidae, Parapaguridae, Albuneidae, Dorippidae, Calappidae, and Pirimelidae. Moreover, no larval descriptions are available in this area for species belonging to the families Bathypalaemonidae, Gnathophyllidae, Ogyrididae, Glyphocrangonidae, Ctenochelidae, Chirostylidae, Cymonomidae, and Palicidae.

\section{ACKNOWLEDGEMENTS}

The authors wish to express their sincere gratitude to P. Nöel and C. d'Udekem d'Acoz for assistance in the preparation of the checklist. The draft was improved by the comments of D.I. Williamson, N. Ngoc-Ho, J.A. Cuesta, G. Guerao, P. Abelló, C. Schubart. Many thanks are due to the libraries of Natural History Museum of London, Paris and Madrid, Plymouth Marine Laboratory, University of Bielefeld, IPIMAR of Lisbon, and CSIC of Cádiz. We also thank A. Fransozo and M.L. NegreirosFransozo for sending us copies of parts of their private libraries. Finally, special thanks to C. Díaz for her help in checking the literatured cited and T. Randsome for his assistance with the final English version.

Part of this work was supported by grants from Consejo Superior de Investigaciones Científicas (CSIC) and Consejería de Medio Ambiente (Junta de Andalucía), Spain.

It is inevitable that there are errors and omissions in this paper; the authors would be grateful for any corrections and additions.

\section{CHECKLIST OF DECAPOD SPECIES FROM GIBRALTAR STRAIT AREA $\left(30^{\circ}-40^{\circ} \mathrm{N}\right.$ TO $\left.0^{\circ}-10^{\circ} \mathrm{W}\right)$ AND CURRENT KNOWLEDGE ON LARVAL DEVELOPMENT}

LU: larvae undescribed; PLD: partial larval description; FLD: full larval description.

Order DECAPODA Latreille, 1802

Subord. DENDROBRANCHIATA Bate, 1888

Superfam. PENAEOIDEA Rafinesque, 1815

Fam. Aristeidae Wood-Mason, 1891

1. Aristaeopsis edwardsiana (Jonhson, 1867)

2. Aristeomorpha foliacea (Risso, 1827)

3. Aristeus antennatus (Risso, 1816)

Fam. Benthysicymidae Wood-Mason, 1891

4. Bentheogennema intermedia (Bate, 1888)

5. Benthesicymus bartletti S.I. Smith, 1882

6. Benthesicymus brasiliensis Bate, 1881

7. Benthonectes filipes S.I. Smith, 1885

8. Gennadas brevirostris Bouvier, 1905

9. Gennadas elegans (S.I. Smith, 1882)

10. Gennadas tinayrei Bouvier, 1906

11. Gennadas valens (S.I. Smith, 1884)

Fam. Penaeidae Rafinesque, 1815

12. Funchalia villosa (Bouvier, 1905)

13. Funchalia woodardii Johnson, 1867

14. Marsupenaeus japonicus (Bate, 1888) ${ }^{1}$

15. Melicertus kerathurus (Forskål, 1775)

16. Parapenaeus longirostris (Lucas, 1846)

17. Penaeopsis serrata (Bate, 1881)

Fam. Solenoceridae Wood-Mason, 1891

18. Hymenopenaeus aphoticus Burkenroad, $1936 \quad$ LU

19. Hymenopenaeus debilis S.I. Smith, 1882 LU

20. Solenocera africana Stebbing, 1917 LU

21. Solenocera membranacea (Risso, 1816) PLD

LU

PLD

Fam. Sicyoniidae Ortmann, 1898

22. Sicyonia carinata (Brünnich, 1768)

FLD

Superfam. SERGESTOIDEA Dana, 1852

LU
LU
LU
LU
LU
PLD
LU
LU

Fam. Sergestidae Dana, 1852

23. Petalidium obesum (Kröyer, 1855) LU

24. Sergestes arcticus Kröyer, 1855 PLD

25. Sergestes armatus Kröyer, $1855 \quad$ PLD

26. Sergestes atlanticus H.M. Edwards, $1830 \quad$ PLD

27. Sergestes cornutus Kröyer, $1855^{2}$ PLD

28. Sergestes curvatus Crosnier and Forest, 1973 PLD

29. Sergestes henseni (Ortmann, 1893) PLD

LU

PLD

FLD

FLD

FLD

LU

${ }^{1}$ Although not its natural habitat, this species may be found here due to the escape of individuals from aquacultural farms.

${ }^{2}$ Adults remain undiscovered. The descriptions of these species are only based on juvenile and larval forms (see Lo Bianco, 1903; Bouvier, 1905; Bernard, 1953). 
30. Sergestes sargassi Ortmann, 1893

31. Sergestes vigilax Stimpson, 1860

32. Sergia japonica Bate, 1881

33. Sergia robusta (S.I. Smith, 1882)

34. Sergia splendens Sund, 1920

35. Sergia tenuiremis Kröyer, 1855

Fam. Luciferidae De Haan, 1849

36. Lucifer typus H.M. Edwards, 1837

Subord. PLEOCYEMATA Burkenroad, 1963 Infraord. STENOPODIDEA Claus, 1872

Fam. Stenopodidae Claus, 1872

37. Odontozona edwardsii (Bouvier, 1908)

38. Spongicoloides evolutus (Bouvier, 1905)

39. Spongicoloides koehleri (Caullery, 1896)

40. Spongicoloides profundus Hansen, 1908

41. Stenopus spinosus Risso, 1827

Infraord. CARIDEA Dana, 1852

Fam. Nematocarcinidae S.I. Smith, 1884

42. Nematocarcinus ensifer (S.I. Smith, 1882)

43. Nematocarcinus exilis Bate, 1888

44. Nematocarcinus gracilipes Filhol, 1884

Fam. Oplophoridae Dana, 1852

45. Acanthephyra brevirostris S.I. Smith, 1885

46. Acanthephyra eximia S.I. Smith, 1884

47. Acanthephyra pelagica (Risso, 1816)

48. Acanthephyra purpurea M. Edwards, 1881

49. Acanthephyra stylorostrata (Bate, 1888)

50. Acanthephyra tenuipes (Bate, 1888)

51. Ephyrina benedicti S.I. Smith, 1885

52. Ephyrina bifida Stephensen, 1923

53. Ephyrina figuerai Crosnier and Forest, 1973

54. Heterogenys microphthalma Smith, 1885

55. Hymenodora gracilis S.I. Smith, 1886

56. Janicella spinicauda (M. Edwards, 1883)

57. Kemphyra corallina (M. Edwards, 1883)

58. Meningodora mollis S.I. Smith, 1882

59. Meningodora vesca S.I. Smith, 1986

60. Notostomus elegans M. Edwards, 1881

61. Oplophorus spinosus (Brullé, 1839)

62. Systelaspis braueri (Balss, 1914)

63. Systelaspis cristata Faxon, 1893

64. Systellaspis debilis (M. Edwards, 1881)

65. Systellaspis pellucida (Filhol, 1885)

Fam. Pasiphaeidae Dana, 1852

66. Eupasiphae gilesii (Wood Mason, 1892)

67. Eupasiphae serrata (Rathbun, 1902)

68. Parapasiphae cristata S.I. Smith, 1884

69. Parapasiphae sulcatifrons S.I. Smith, 1884

70. Pasiphaea hoplocerca Chace, 1940

71. Pasiphaea multidentata Esmark, 1866

72. Pasiphaea sivado (Risso, 1816)

73. Pasiphaea tarda Kröyer, 1865

74. Psathyrocaris fragilis Wood Mason, 1893

75. Psathyrocaris infirma Alcock and Anderson, 1894

LU

LU

LU

LU

FLD

FLD

PLD

PLD

LU

PLD

FLD

LU

PLD

LU

LU

LU

PLD

FLD

PLD

LU

LU

LU

LU

LU

LU

LU

LU

LU

LU

PLD

LU

LU

FLD

LU

Fam. Bathypalaemonidae Chace, 1992

76. Bathypalaemonella serratipalma Pequegnat, 1970

Fam. Gnathophyllidae Dana, 1852

77. Gnathophyllum elegans (Risso, 1816)

\section{LU}

LU

LU

PLD

LU

PLD

FLD

PLD

LU

Fam. Palaemonidae Rafinesque, 1815

78. Balssia gasti (Balss, 1921)

79. Brachycarpus biunguiculatus (Lucas, 1846)

80. Palaemon adspersus Rathke, 1837

81. Palaemon elegans Rathke, 1837

82. Palaemon longirostris M. Edwards, 1837

83. Palaemon serratus (Pennant, 1777)

84. Palaemon xiphias Risso, 1816

85. Palaemonetes varians (Leach, 1814)

86. Palaemonetes zariquieyi Sollaud, 1939

FLD

FLD

FLD

FLD

FLD

FLD

FLD

FLD
87. Periclimenes amethysteus (Risso, 1827)

88. Periclimenes kornii (Lo Bianco, 1903)

89. Periclimenes sagittifer (Norman, 1861)

90. Periclimenes scriptus (Risso, 1822)

91. Pontonia flavomaculata Heller, 1864

92. Pontonia pinnophylax (Otto, 1821)

93. Typton spongicola Costa, 1844

LU

LU

LU

LU

FLD

FLD

PLD

Fam. Alpheidae Rafinesque, 1815

94. Alpheus dentipes Meneville, 1832

95. Alpheus glaber (Olivi, 1792)

96. Alpheus macrocheles (Hailstone, 1835)

97. Alpheus platydactylus Coutière, 1897

98. Alpheus talismani Coutière, 1898

99. Athanas amazone Holthuis, 1951

100. Athanas grimaldii Coutière, 1911

101. Athanas nitescens (Leach, 1814)

102. Synalpheus gambarelloides (Nardo, 1847)

103. Synalpheus tumidomanus africanus Crosnier and Forest, 1965

LU

FLD

FLD

LU

LU

LU

LU

PLD

PLD

LU

Fam. Hippolytidae Dana, 1852

104. Eualus drachi Nöel, 1978

105. Eualus lebourae Holthuis, 1951

106. Eualus occultus (Lebour, 1936)

107. Hippolyte garciarasoi d'Udekem d'Acoz, 1996

108. Hippolyte holthuisi Zariquiey, 1953

109. Hippolyte inermis Leach, 1815

110. Hippolyte lagarderei d'Udekem d'Acoz, 1995

111. Hippolyte leptocerus (Heller, 1863)

113. Hippolyte niezabitowskii d'Udeken d'Acoz, 1996 LU

114. Hippolyte varians Leach, $1814 \quad$ PLD

115. Ligur ensiferus (Risso, 1816)

116. Lysmata seticaudata (Risso, 1816)

117. Lysmata uncicornis Holthuis and Maurin, 1952

118. Merhippolyte ancistrota Crosnier and Forest, 1973

119. Thoralus cranchii (Leach, 1817)

120. Thoralus sollaudi Zariquiey, 1935

LU

LU

LU

LU

PLD

LU

U

Fam. Ogyrididae Stebbing, 1914

121. Ogyrides rarispina Holthuis, 1951

LU

FLD

LU

LU

FLD

LU

Fam. Processidae Ortmann, 1896

122. Processa acutirostris Nouvel and Holthuis, 1957 LU

123. Processa canaliculata Leach, 1815 PLD

124. Processa edulis (Risso, 1816) PLD

125. Processa elegantula Nouvel and Holthuis, 1957 PLD

126. Processa intermedia Holthuis, 1951

127. Processa macrodactyla Holthuis, 1952

Holthuis, 1957 LU

130. Processa nouveli Al-Adhub and Williamson, 1975 FLD

131. Processa robusta Nouvel and Holthuis, 1957

Fam. Pandalidae Haworth, 1825

132. Chlorotocus crassicornis (Costa, 1871)

133. Dichelopandalus bonnieri (Caullery, 1896)

134. Heterocarpus ensifer M. Edwards, 1881

PLD

PLD

135. Heterocarpus grimaldii M. Edwards and Bouvier, 1900 LU

136. Pandalina brevirostris (Rathke, 1843)

137. Pandalina profunda Holthuis, 1946

138. Parapandalus narval (Fabricius, 1787)

139. Plesionika acanthonotus (S.I. Smith, 1882)

140. Plesionika antigai Zariquiey, 1955

141. Plesionika edwardsii (Brandt, 1851)

142. Plesionika ensis (A.M. Edwards, 1881)

143. Plesionika giglioli (Senna, 1908)

144. Plesionika heterocarpus (Costa, 1871)

145. Plesionika martia M. Edwards, 1883

146. Plesionika williamsi Forest, 1964

147. Stylopandalus richardi (Coutière, 1905)

LU

LU

PLD

LU

PLD

LU

LU

LU

PLD

Fam. Crangonidae Haworth, 1825

148. Aegaeon cataphractus (Olivi, 1792)

149. Aegaeon lacazei (Gourret, 1887)

150. Crangon crangon (Linnaeus, 1758)

151. Parapontophilus abyssi (S.I. Smith, 1884)
PLD

PLD

FLD 
152. Parapontophilus talismani (Crosnier and Forest, 1973) LU

153. Philocheras bispinosus bispinosus (Hailstone, 1835) PLD

154. Philocheras bispinosus neglectus (Sars, 1883) FLD

155. Philocheras echinulatus (Sars, 1861)

156. Philocheras fasciatus (Risso, 1816)

157. Philocheras monacanthus (Holthuis, 1961)

158. Philocheras sculptus (Bell, 1847)

159. Philocheras trispinosus (Hailstone, 1835)

160. Pontophilus norvegicus (Sars, 1861)

161. Pontophilus spinosus (Leach, 1815)

Fam. Glyphocrangonidae S.I. Smith, 1884

162. Glyphocrangon longirostris (S.I. Smith, 1882)

163. Glyptocrangon sculpta (S.I. Smith, 1882)

PLD

FLD

FLD

PLD

FLD

PLD

PLD

\section{LU}

LU

Infraord. ASTACIDEA Latreille, 1803

Fam. Nephropidae Dana, 1852

164. Homarus gammarus (Linnaeus, 1758)

165. Nephrops norvegicus (Linnaeus, 1758)

166. Nephropsis atlantica Norman, 1882

Infraord. THALASSINIDEA Latreille, 1831

Fam. Axiidae Huxley, 1879

167. Axius stirhynchus Leach, 1815

168. Calocarides coronatus (Trybom, 1904)

PLD

PLD

Fam. Calocarididae Ortmann, 1891

169. Calocaris macandreae Bell, 1846

PLD

Fam. Callianassidae Dana, 1852

170. Callianassa candida (Olivi, 1792)

171. Callianassa subterranea (Montagu, 1808)

172. Callianassa tyrrhena (Petagna, 1792)

173. Necallianassa truncata Giard and Bonnier, 1890

LU

FLD

PLD

LU

Fam. Ctenochelidae Manning and Felder, 1991

174. Gourretia denticulata (Luzte, 1937)

LU

Fam. Laomediidae Borradaile, 1903

175. Jaxea nocturna Nardo, 1847

Fam. Upogebiidae Borradaile, 1903

176. Gebiacantha talismani (Bouvier, 1915)

177. Upogebia deltaura (Leach, 1815)

178. Upogebia pusilla (Petagna, 1792)

179. Upogebia tipica (Nardo, 1869)

Infraord. PALINURA Latreille, 1902

Fam. Polychelidae Wood Mason, 1875

180. Eryoneicus puritanii Lo Bianco, $1903^{1}$

181. Eryoneicus spinoculatus Bouvier, $1905^{1}$

182. Polycheles crucifer (Willemoes-Suhm, 1874)

183. Polycheles granulatus Faxon, 1893

184. Polycheles typhlops Heller, 1862

185. Polycheles validus (M. Edwards, 1880)

186. Stereomastis nana (S.I. Smith, 1884)

187. Stereomastis sculpta (S.I. Smith, 1880)

FLD

LU

FLD

FLD

LU

PLD

LU

genidae Ortmann, 1892

194. Calcinus tubularis (Linnaeus, 1767)

195. Clibanarius erythropus (Latreille, 1818)

FLD

196. Dardanus arrosor (Herbst, 1796)

197. Dardanus calidus (Risso, 1826)

198. Diogenes pugilator (Roux, 1829)

199. Paguristes eremita (Linnaeus, 1767)

FLD

FLD

PLD

FLD

Fam. Paguridae Latreille, 1803

200. Anapagurus alboranensis García-Gómez, $1994 \quad$ LU

201. Anapagurus bicorniger M. Edwards and Bouvier, 1892 FLD

202. Anapagurus breviaculeatus Fenizia, 1937 LU

203. Anapagurus chiroacanthus (Lilljeborg, 1856) FLD

204. Anapagurus hyndmanni (Bell, 1845) FLD

205. Anapagurus laevis (Bell, 1846) FLD

206. Anapagurus longispina M. Edwards and Bouvier, 1900 LU

207. Anapagurus petiti Dechancé and Forest, 1962 PLD

208. Anapagurus pusillus Henderson, 1888 LU

209. Catapaguroides iris Bouvier, 1922 LU

210. Catapaguroides megalops $\mathrm{M}$. Edwards and Bouvier, 1892

LU

211. Catapaguroides microps M. Edwards and Bouvier, 1892 LU

212. Cestopagurus timidus (Roux, 1830)

213. Michelopagurus richardi (Bouvier, 1922)

214. Nematopagurus longicornis M. Edwards and Bouvier, 1892

215. Pagurus alatus (Fabricius, 1775)

216. Pagurus anachoretus Risso, 1826

217. Pagurus bernhardus (Linnaeus, 1758)

218. Pagurus carneus Pocock, 1889

219. Pagurus chevreuxi (Bouvier, 1896)

220. Pagurus cuanensis Bell, 1845

221. Pagurus excavatus (Herbst, 1791)

222. Pagurus forbesii Bell, 1845

223. Pagurus prideaux Leach, 1815

224. Pagurus pubescentulus (M. Edwards and Bouvier, 1892)

225. Spiropagurus elegans Miers, 1881

FLD

LU

PLD

PLD

FLD

FLD

PLD

LU

FLD

FLD

PLD

FLD

Fam. Parapaguridae S.I. Smith, 1882

226. Oncopagurus bicristatus (M. Edwards, 1880)

227. Paragiopagurus ruticheles (M. Edwards, 1891)

228. Parapagurus pilosimanus S.I. Smith, 1879

229. Strobopagurus gracilipes (M. Edwards, 1891)

230. Sympagurus acinops (Lemaitre, 1989)

LU

FLD

Fam. Chirostylidae Ortmann, 1892

LU

LU

PLD

LU

LU

PLD 234. Galathea bolivari Zariquiey, 1950

PLD 235. Galathea cenarroi Zariquiey, 1968

Fam. Palinuridae Latreille, 1802

188. Palinurus elephas (Fabricius, 1787)

189. Palinurus mauritanicus Gruvel, 1911

FLD

$\mathrm{LU}$

Fam. Scyllaridae Latreille, 1825

190. Scyllarides latus (Latreille, 1803)

191. Scyllarus arctus (Linnaeus, 1758)

192. Scyllarus posteli Forest, 1963

193. Scyllarus pygmaeus (Bate, 1888)

PLD

FLD

PLD

PLD

Infraord. ANOMURA MacLeay, 1838

${ }^{1}$ Adults remain undiscovered. The descriptions of these species are only based on juvenile and larval forms (see Lo Bianco, 1903; Bouvier, 1905; Bernard, 1953).
231. Gastroptychus formosus (Filhol, 1884)

232. Uroptychus bouvieri Caullery, 1896

233. Uroptychus marrocanus Türkay, 1976

Fam. Galatheidae Samouelle, 1819

236. Galathea dispersa Bate, 1859

237. Galathea faiali Nunes Ruivo, 1961

238. Galathea intermedia Lilljeborg, 1851

239. Galathea nexa Embleton, 1834

240. Galathea squamifera Leach, 1814

241. Galathea strigosa (Linnaeus, 1767)

242. Munida curvimana M. Edwards and Bouvier, 1894

243. Munida intermedia M. Edwards and Bouvier, 1899

244. Munida microphthalma M. Edwards, 1880

245. Munida rugosa (Fabricius, 1775)

246. Munida rutllanti Zariquiey, 1952

247. Munida sarsi Huus, 1935

248. Munida tenuimana Sars, 1872

249. Munidopsis curvirostra Whiteaves, 1874

250. Munidopsis serricornis (Lovén, 1853)

LU

LU

LU

Fam. Porcellanidae Haworth, 1825

251. Pisidia bluteli (Risso, 1816)

252. Pisidia longicornis (Linnaeus, 1767)

253. Porcellana platycheles (Pennant, 1777)

LU

LU

FLD

LU

FLD

PLD

FLD

FLD

LU

PLD

LU

PLD

LU

PLD

FLD

LU

FLD

Fam. Albuneidae Stimpson, 1858

254. Albunea carabus (Linnaeus, 1758)

PLD

FLD

FLD

PLD 
Infraord. BRACHYURA Latreille, 1802

Fam. Dromiidae De Haan, 1833

255. Dromia personata (Linnaeus, 1758)

Fam. Cymonomidae Bouvier, 1898

256. Cymonomus granulatus (Thomson, 1873)

Fam. Homolidae de Haan, 1839

257. Homola barbata (Fabricius, 1793)

258. Homologenus boucheti Guinot and Richer de Forges, 1995

259. Paromola cuvieri (Risso, 1816)

LU

PLD

Fam. Latreillidae Stimpson, 1858

260. Latreillia elegans Roux, 1830

PLD

Fam. Dorippidae MacLeay, 1838

261. Ethusa mascarone (Herbst, 1785)

262. Ethusina talismani M. Edwards and Bouvier, 1897

263. Medorippe lanata (Linnaeus, 1767)

Fam. Calappidae De Haan, 1833

264. Calappa granulata (Linnaeus, 1758)

265. Cryptosoma cristata (Brullé, 1837)

Fam. Leucosiidae Samouelle, 1819

266. Ebalia cranchii Leach, 1817

267. Ebalia deshayesi Lucas, 1846

268. Ebalia edwardsi Costa, 1838

269. Ebalia granulosa H.M. Edwards, 1837

270. Ebalia nux M. Edwards, 1883

271. Ebalia tuberculata Miers, 1881

272. Ebalia tuberosa (Pennant, 1777)

273. Ebalia tumefacta (Montagu, 1808)

274. Ilia nucleus (Linnaeus, 1758)

275. Merocryptus boletifer M. Edwards and Bouvier, 1894

Fam. Majidae Samouelle, 1819

276. Acanthonyx lunulatus (Risso, 1816)

277. Achaeus cranchii Leach, 1817

278. Achaeus gracilis Costa, 1839

279. Dorhynchus thomsoni Thomson, 1873

280. Ergasticus clouei M. Edwards, 1882

281. Eurynome aspera (Pennant, 1777)

282. Eurynome spinosa Hailstone, 1835

283. Herbstia condyliata (Fabricius, 1787)

284. Inachus aguiarii Brito Capello, 1876

285. Inachus communissimus Rizza, 1839

286. Inachus dorsettensis (Pennant, 1777)

287. Inachus leptochirus Leach, 1817

288. Inachus phalangium (Fabricius, 1775)

289. Inachus thoracicus (Roux, 1830)

290. Lissa chiragra (Fabricius, 1775)

291. Macropodia czerniawskii (Brandt, 1880)

292. Macropodia deflexa Forest, 1978

293. Macropodia intermedia Bouvier, 1940

294. Macropodia linaresi Forest and Zariquiey, 1964

295. Macropodia longipes (A.M. Edwards and Bouvier, 1899)

296. Macropodia longirostris (Fabricius, 1775)

297. Macropodia parva Van Noort and Adema, 1985

298. Macropodia rostrata (Linnaeus, 1761)

299. Macropodia tenuirostris (Leach, 1814)

300. Maja brachydactyla Balss, 1922

301. Maja crispata (Risso, 1827)

302. Maja goltziana d'Oliveira, 1888

303. Maja squinado (Herbst, 1788)

304. Pisa armata (Latreille, 1803)

305. Pisa carinimana Miers, 1879

306. Pisa muscosa (Linnaeus, 1758)

307. Pisa nodipes (Leach, 1815)

308. Pisa tetraodon (Pennant, 1777)

309. Rochinia carpenteri (Thomson, 1873)

310. Rochinia rissoana (Roux, 1828)

Fam. Parthenopidae MacLeay, 1838

PLD

LU

LU

FLD

LU

LU

PLD

LU

FLD

FLD
FLD

311. Heterocrypta maltzami Miers, 1881

PLD

312. Parthenope angulifrons Latreille, 1825

313. Parthenope expansa (Miers, 1879)

314. Parthenope macrochelos (Herbst, 1790)

PLD

315. Parthenope massena (Roux, 1830)

LU

PLD

316. Parthenope miersi (Milne Edwards and Bouvier, 1898) LU

Fam. Atelecyclidae Ortmann, 1893

317. Atelecyclus rotundatus (Olivi, 1792)

318. Atelecyclus undecimdentatus (Herbst, 1783)

FLD

Fam. Cancridae Latreille, 1803

319. Cancer bellianus Johnson, 1861

320. Cancer pagurus Linnaeus, 1758

Fam. Corystidae Samouelle, 1819

321. Corystes cassivelaunus (Pennant, 1777)

PLD

FLD

Fam. Pirimelidae Alcock, 1819

322. Pirimela denticulata (Montagu, 1808)

323. Sirpus zariquieyi Gordon, 1953

Fam. Thiidae Dana, 1852

324. Thia scutellata (Fabricius, 1793)

FLD

PLD Fam. Geryonidae Colosi, 1923

325. Chaceon inglei Manning and Holthuis, 1989

326. Geryon longipes M. Edwards, 1882

327. Geryon trispinosus (Herbst, 1803)

LU

FLD

Fam. Portunidae Rafinesque, 1815

328. Bathynectes longipes (Risso, 1816)

329. Bathynectes maravigna (Pestandrea, 1839)

330. Carcinus aestuarii Czerniavsky, 1884

331. Carcinus maenas (Linnaeus, 1758)

332. Liocarcinus arcuatus (Leach, 1814)

333. Liocarcinus bolivari (Zariquiey, 1948)

334. Liocarcinus corrugatus (Pennant, 1777)

335. Liocarcinus depurator (Linnaeus, 1758)

336. Liocarcinus holsatus (Fabricius, 1798)

337. Liocarcinus maculatus (Risso, 1827)

338. Liocarcinus marmoreus (Leach, 1814)

339. Liocarcinus mcleayi (Barnard, 1947)

340. Liocarcinus pusillus (Leach, 1815)

341. Liocarcinus vernalis (Leach, 1815)

342. Liocarcinus zariquieyi (Gordon, 1968)

343. Macropipus tuberculatus (Roux, 1830)

344. Necora puber (Linnaeus, 1767)

345. Polybius henslowii Leach, 1820

346. Portumnus latipes (Pennant, 1777)

347. Portunus hastatus (Linnaeus, 1767)

348. Xaiva biguttata (Risso, 1816)

Fam. Goneplacidae MacLeay, 1838

349. Goneplax rhomboides (Linnaeus, 1758)

FLD

PLD

FLD

LU

FLD

FLD

FLD

LU

FLD

FLD

FLD

LU

FLD

LU

FLD

PLD

LU

PLD

FLD

PLD

FLD

LU

PLD

FLD

Fam. Xanthidae Dana, 1851

350. Eriphia verrucosa (Forskål, 1775)

351. Monodaeus couchi (Couch, 1851)

352. Monodaeus guinotae Forest, 1976

353. Panopeus africanus M. Edwards, 1867

354. Paractea monodi Guinot, 1969

355. Pilumnus hirtellus (Linnaeus, 1761)

356. Pilumnus inermis M. Edwards and Bouvier, 1894

357. Pilumnus spinifer H.M. Edwards, 1834

358. Pilumnus villosissimus (Rafinesque, 1814)

359. Rhithropanopeus harrisii (Gould, 1841)

360. Xantho incisus (Leach, 1814)

361. Xantho pilipes M. Edwards, 1867

362. Xantho poressa (Olivi, 1792)

FLD

FLD

LU

LU

FLD

LU

PLD

LU

FLD

FLD

PLD

FLD

Fam. Grapsidae MacLeay, 1838

363. Brachynotus atlanticus Forest, 1957

364. Brachynotus sexdentatus (Risso, 1827)

365. Euchirograpsus liguricus M. Edwards, 1853

366. Grapsus adscensionis (Osbeck, 1765)

367. Pachygrapsus marmoratus (Fabricius, 1787)

368. Pachygrapsus maurus (Lucas, 1846)

369. Pachygrapsus transversus (Gibbes, 1850)

FLD

LU

PLD

FLD

PLD

PLD 
370. Percnon gibbesi (H.M. Edwards, 1853)

371. Plagusia depressa (Fabricius, 1775)

372. Planes minutus (Linnaeus, 1758)

Fam. Pinnotheridae De Haan, 1833

373. Asthenognathus atlanticus Monod, 1933

374. Neopinnotheres pinnotheres (Linnaeus, 1758)
375. Pinnotheres pisum (Linnaeus, 1767)

FLD

Fam. Ocypodidae Rafinesque, 1815

376. Uca tangeri (Eydoux, 1835)

Fam. Palicidae Rathbun, 1898

FLD 377. Palicus caronii (Roux, 1830)
FLD

LU

\section{ANNOTATED BIBLIOGRAPHY OF DECAPOD LARVAE}

Order DECAPODA Latreille, 1802

Oxyrhynchi: Cano, G. 1893a. CG.

Decapoda: Gurney, R. 1942. CG.

Decapoda: Williamson, D.I. 1957. CG. K.

Decapoda: Barnich, R. 1996. CG. K.

Suborder DENDROBRANCHIATA Bate, 1888

Dendrobranchiata: Santos, A. dos; Lindley, J.A. 2000. CG. K.

Superfamily PENAEOIDEA Rafinesque, 1815

Penaeidea: Gurney, R. 1942. CG.

Penaeoidea: Boschi, E.E. 1981. CG.

Peneides: Seridji, R. 1989. PT.Y1. K.

Family Aristeidae Wood-Mason, 1891 Aristeidae: Dall, W.; Hill, B.J.; Rothlisberg, P.C.; Staples, D.J. 1990. PT.Y.PL. (B). K. Aristeidae: Barnich, R. 1996. K.

Aristaeomorpha foliacea (Risso, 1827) Aristaeomorpha foliacea: Heldt, J.H. 1954. Z1-Z4. (P). Aristaeomorpha foliacea: Heldt, J.H. 1955a. PZ2-3.Y1.LS. $(\mathrm{P}+\mathrm{L}+\mathrm{R})$.

Aristeus antennatus (Risso, 1816) Aristeus antennatus: Heldt, J.H. 1954. Z1-Z4. (P). Aristeus antennatus: Heldt, J.H. 1955a. PZ1-PZ3.Y1. (P+L+R). Aristeus antennatus: Seridji, R. 1971. (P+B). K.

Family Benthysicymidae Wood-Mason, 1891 ? Aristeus: Bate, C.S. 1888. M. (P).

Benthysicymus $s p$.

? Benthysicymus: Gurney, R. 1924a. Y1. (P).

Gennadas sp.

Penaeus larve: Claus, C. 1876. PZ. Z. Y. (P).

Gennadas: Gurney, R. 1924a. PZ1-PZ3.Y1-Y4. (P). Gennadas: Gurney, R. 1942. CG

? Gennadas valens: Criales, M.M.; McGowan, M.F. 1993.

Y1-Y4. (P).

Gennadas elegans (S.I. Smith, 1882)

Euphema armata: Ortmann, A. 1893. NE. (P) CG.

Amalopenaeus elegans: Monticelli, F.S.; Lo Bianco, S. 1900.

Amalopenaeus elegans: Lo Bianco, S. 1901. NE. (P). Amalopenaeus elegans: Williamson, H.C. 1915. CG. (P).

? Euphema armata: Stephensen, K. 1923. NE. (P). $(\mathrm{P}+\mathrm{R})$

* Gennadas elegans: Heldt, J.H. 1938. PT2.PT3.Y1.Y2.Y4.

Gennadas elegans: Gurney, R. 1942. PZ2.Y1. (P).

Gennadas elegans: Gurney, R. 1943. PT. (B).

Gennadas elegans: Kurian, C.V. 1956. Y1-Y5. (P).

Gennadas elegans: Seridji, R. 1971. (B). K.

Gennadas elegans: Seridji, R. 1989. K.

Gennadas elegans: Barnich, R. 1996. PZ1-PZ3.Z1-Z4. (P+B). K.

Family Penaeidae Rafinesque, 1815

Penaeus larva No. 3: Stephensen, K. 1923. NE. (P)

Pénéides: Heldt, J.H. 1938. CG.

Penaeidae: Dall, W.; Hill, B.J.; Rothlisberg, P.C.; Staples, D.J.

1900. PT.Y.PL. (B). K.

Funchalia sp.

Funchalia: Dall, W.; Hill, B.J.; Rothlisberg, P.C.; Staples, D.J.
1990. Y. (B). K.

Funchalia woodwardii Johnson, 1867

(P). Aristeus antennatus: Monticelli, F.S.; Lo Bianco, S. 1902. Y?

Aristaeomorpha foliacea: Stephensen, K. 1923. LS. (P). Aristaeomorpha foliacea: Kurian, C.V. 1956. Y4? (P). Aristaeomorpha foliacea: Paulinose, V.T. 1984. LS. (P). Funchalia woodwardi: Barnich, R. 1996. K.

Melicertus kerathurus (Forskål, 1775) * Penaeus trisulcatus: Heldt, J.H. 1938. L1-L8.PT1-PT3.Y1-

Y4. (L).

Penaeus kerathurus: Kurian, C.V. 1956. PT2. (P).

Penaeus trisulcatus: Al-Kholy, A.A.; El-Hawary, M.M. 1970.

L1-L3.PT1-PT3.PL. (L+P).

Penaeus trisulcatus: Seridji, R. 1971. (B). K.

Penaeus kerathurus: Lumare, F.; Gozzo, S. 1973. L1-L6. (L). Penaeus kerathurus: Barnich, R. 1996. K.

Marsupenaeus japonicus (Bate, 1888)

* Penaeus japonicus: Hudinaga, M. 1942. L1-L6.PT1-

PT3.Y1-Y3.PL. (L)

Penaeus japonicus: Al-Kholy, A.A.; El-Hawary, M.M. 1970.

PT1-PT3.Y2. (P)

Parapenaeus sp.

Parapenaeus sp.: Gurney, R. 1942. CG.

(P).

Parapenaeus ASM1: Rice, A.L.; Williamson, D.I. 1977. Z5?.

Parapenaeus: Dall, W.; Hill, B.J.; Rothlisberg, P.C.; Staples,

D.J. 1990. PT1-PT3.Y.PL. (B). K.

Parapenaeus longirostris (Lucas, 1846)

* Parapenaeus longirostris: Heldt, J.H. 1938. L1-L8.PT1-

PT3.Y1-Y4. (L).

Parapenaeus longirostris: Gurney, R. 1942. CG.

Parapenaeus longirostris: Gurney, R. 1943. PT. (B).

Parapenaeus longirostris: Seridji, R. 1971. K.

Parapenaeus longirostris: Barnich, R. 1996. K.

Penaeopsis sp.

Penaeopsis: Gurney, R. 1924a. Y. (P).

? Penaeopsis stebbingi: Gurney, R. 1927. L1-L3.PT1-PT3.Y1-

Y3. (P).

Penaeopsis sp.: Gurney, R. 1943. PT1-PT3.Z1-Z3.PL. (P).

Penaeus sp.

Penaeus: Brooks, W.K. 1882. L.PT.S. (B).

Penaeus: Gurney, R. 1942. CG.

Penaeus: Dall, W.; Hill, B.J.; Rothlisberg, P.C.; Staples, D.J.

1990. PT.Y.PL. (B). K.

Family Solenoceridae Wood-Mason, 1891

Solenoceridae: Dall, W.; Hill, B.J.; Rothlisberg, P.C.; Staples, D.J. 1990. PT.Y.PL. (B). K.

Solenocera sp.

Solenocera: Gurney, R. 1942. CG.

Solenocera membranacea (Risso, 1816)

Solenocera siphonocera: Monticelli, F.S.; Lo Bianco, S. 1900. CG.

Penaeus membranaceus: Monticelli, F.S.; Lo Bianco, S. 1901. CG. Penaeus siphonocerus: Williamson, H.C. 1915. L.PT.Y. (P). $(\mathrm{P}+\mathrm{R})$ 
Solenocera membranacea: Heldt, J.H. 1955. PT1-

PT3.Y1.Y2.PL. (P).

Solenocera membranacea: Kurian, C.V. 1956. PT2.PT3. Y1. Y2. (P).

Solenocera membranacea: Heegaard, P. 1966. Z1-Z7. (P).

Solenocera membranacea: Seridji, R. 1971. K.

Solenocera membranacea: Seridji, R. 1989. K.

Solenocera membranacea: Barnich, R. 1996. PZ1-PZ3.Z1.Z2. (P). K.

Family Sicyoniidae Ortmann, 1898

Sicyoniidae: Dall, W.; Hill, B.J.; Rothlisberg, P.C.; Staples, D.J. 1990. PT.Y.PL. (B). K.

Sicyonia sp.

Sicyonia: Gurney, R. 1942. CG.

Sicyonia carinata (Brünnich, 1768)

Sicyonia sculpta: Monticelli, F.S.; Lo Bianco, S. 1900. Z1-Z3. (P).

* Sicyonia carinata: Heldt, J.H. 1938. L1-L8.PT1-PT3.Y1-Y4. (L).

Sicyonia carinata: Gurney, R. 1943. PT. (B).

Sicyonia carinata: Kurian, C.V. 1956. Y1. (P).

Sicyonia carinata: Seridji, R. 1971. K.

Sicyonia carinata: Seridji, R. 1989. K.

$\mathrm{K}$.

Sicyonia carinata: Barnich, R. 1996. PZ1-PZ3.Z1-Z4. (P+B).

Superfamily SERGESTOIDEA Dana, 1852

Sergestoidea: Boschi, E.E. 1981. CG.

Family Sergestidae Dana, 1852

Sergestidae: Dall, W.; Hill, B.J.; Rothlisberg, P.C.; Staples,

D.J. 1990. PT.Y.PL. (B). K.

Sergestidae: Barnich, R. 1996. PL. K.

Sergestes $s p$.

Sergestes: Brooks, W.K. 1882. PT1.E.A.T. (B).

Sergestes: Hansen, H.J. 1922. CG.

Sergestes: Gurney, R.; Lebour, M.V. 1940. K.

Sergestes corniculum form C: Gurney, R.; Lebour, M.V.

1940. A2.T1. (P).

Sergestes: Gurney, R. 1942. CG.

Sergestes: Barnich, R. 1996. PZ1-PZ3.Z1.Z2. CG. (P). K.

Sergestes arcticus Kröyer, 1855

Sergestes arcticus: Dohrn, A. 1908. PT3. (P).

Sergestes arcticus: Wasserloos, E. 1908. PT1-PT3.A.T. (P).

(P).

Sergestes arcticus: Williamson, H.C. 1915. PT1.PT2.Z.A.T

Sergestes arcticus: Hansen, H.J. 1922. PT1.PT2.E.A.T. (P). Sergestes arcticus: Gurney, R.; Lebour, M.V. 1940.

E2.E3.A2.T1. (P).

Sergestes arcticus: Kurian, C.V. 1956. E2.E3.A1.A2.T1.T2.

(P). K.

Sergestes arcticus: Boschi, E.E. 1981. E. (B). CG.

Sergestes arcticus: Barnich, R. 1996. K.

Sergestes armatus Kröyer, 1855

Sergestes armatus: Hansen, H.J. 1922. T. (P).

Sergestes diaponticus: Illig, G. 1927. PZ. (P).

Sergestes armatus: Cecchini, C. 1928. M. (P)

* Sergestes armatus: Gurney, R.; Lebour, M.V. 1940. E1-

E3.A1.A2.T1. (P+R).

Sergestes armatus: Heldt, J.H. 1955. A2. (B).

Sergestes atlanticus $\mathrm{H}$. Milne Edwards, 1830

Sergestes atlanticus: Illig, G. 1914. Z5. (P)

Sergestes atlanticus: Hansen, H.J. 1922. T.

Sergestes atlanticus: Gurney, R.; Lebour, M.V. 1940. Z1-

Z5.M. (P+R).

Sergestes atlanticus: Gurney, R. 1942. A2. (B).

Sergestes atlanticus: Heldt, J.H. 1955. E1. (B).

Sergestes atlanticus: Barnich, R. 1996. Z1.Z2.PL. (P). K.

Sergestes cornutus Köyer, 1855

Sergestes cornutus: Sund, O. 1920 NE. (P).
Sergestes curvatus Crosnier and Forest, 1973

Sergestes corniculum form A: Gurney, R.; Lebour, M.V. 1940. E1-E3.A1.A2.T1. (P+R).

Sergestes henseni (Ortmann, 1893)

Sergestes corniculum: Hansen, H.J. 1922. T.A. (P).

Sergestes corniculum: Gurney, R. 1924a. PT1-PT3. (P).

Sergestes corniculum form B: Gurney, R.; Lebour, M.V.

1940. A2. (P).

Sergestes corniculum: Kurian, C.V. 1956. A1. (P). K.

Sergestes henseni: Barnich, R. 1996. K.

Sergestes sargassi Ortmann, 1893

Sergestes sargassi: Ortmann, A. 1893. A. (P).

Sergestes sargassi: Hansen, H.J. 1922. T. (P).

Sergestes nudus: Illig, G. 1927. T. (P).

Sergestes sargassi: Cecchini, C. 1928. M. (P).

Sergestes sargassi: Gurney, R.; Lebour, M.V. 1940. A2.T2?. (P).

Sergestes sargassi: Kurian, C.V. 1956. A1.A2. (P). K.

Sergestes sargassi: Barnich, R. 1996. K.

Sergestes tenuiremis (Kröyer, 1855)

Sergestes tenuiremis: Hansen, H.J. 1922. T.A. (P).

Sergestes tenuiremis: Gurney, R.; Lebour, M.V. 1940. Z2-

Z5.M2?. (P+R).

Sergestes vigilax Stimpson, 1860

Sergestes: Claus, C. 1876. E.A. (P)

Sergestes vigilax: Hansen, H.J. 1922. T.A. (P).

Sergestes vigilax: Gurney, 1924a. PZ2.PZ3. (P)

Sergestes diaponticus: Illig, G. 1927. E. (P).

Sergestes vigilax: Cecchini 1928. Z4.Z5. (P).

* Sergestes vigilax: Gurney, R.; Lebour, M.V. 1940. E1-

E3.A1.A2.T1.T2. (P+R).

Sergestes vigilax: Heldt, J.H. 1955. A2. (B).

K.

Sergestes vigilax: Kurian, C.V. 1956. E3.A1.A2.T2?.T?. (P).

Sergestes vigilax: Barnich, R. 1996. K.

Sergia japonica Bate, 1881

Sergestes robustus form C: Gurney, R.; Lebour, M.V. 1940.

E1-E3.A2. (P).

Sergia robusta (S.I. Smith, 1882)

Sergestes robustus: Williamson, H.C. 1915. CG. (P).

Sergestes robustus: Hansen, H.J. 1922. T.A. (P)

Sergestes arcticus: Illig, G. 1927. A. (P).

* Sergestes robustus: Gurney, R.; Lebour, M.V. 1940.

E2.E3.A1.A2.T1.T2. (P).

Sergestes robustus: Gurney, R. 1942. T. (B).

Sergestes robustus: Heldt, J.H. 1955. E2.E3.A2. (B).

Sergestes robustus: Kurian, C.V. 1956. E2.E3.A1.A2.T1.T2. (P). Sergestes robustus: Barnich, R. 1996. PT2.PT3.Z1.Z2. K.

Family Luciferidae Dana, 1852

Lucifer: Hashizume, K. 1999. CG.

Lucifer $s p$.

Leucifer larve: Claus, C. 1876. PZ2. (P).

Leucifer: Brooks, W.K. 1880. L1. (L).

Leucifer typus: Williamson, H.C. 1915. Z1-Z8. (P).

Lucifer typus: Barnich, R. 1996. PZ1-PZ3.Z1-Z3.PL. (B). K.

Lucifer typus $\mathrm{H}$. Milne Edwards, 1837

$\mathrm{K}$.

* Lucifer typus: Hashizume, K. 1999. PZ1-PZ3.Z1.Z2.M1. (P).

Suborder PLEOCYEMATA Burkenroad, 1963

Infraorder STENOPODIDEA Claus, 1872

Family Stenopodidae Claus, 1872

Stenopus sp.

Stenopus: Gurney, R. 1942. CG.

Stenopus spinosus Risso, 1827

Stenopus spinosus: Cano, G. 1891b. Z1.Z3.PS. (P). 
Stenopus spinosus: Kurian, C.V. 1956. Z1.Z2. (P). Stenopus spinosus: Bourdillon Casanova, L. 1960. Z1. (P). Stenopus spinosus: Seridji, R. 1989. Z1-Z5. (P).

* Stenopus spinosus: Seridji, R. 1990. Z1-Z5. (P).

Stenopus spinosus: Barnich, R. 1996. Z1-Z5. (P+B). K.

Infraorder CARIDEA Dana, 1852

Caridea: Gurney, R. 1942. CG.

Caridea: Boschi, E.E. 1981. CG.

Family Nematocarcinidae S.I. Smith, 1884

Nematocarcinus exilis Bate, 1888

Larva allied to Caricyphus: Kemp, S.W. 1907. NE. (P). Nematocarcinus ensifer exilis: Kemp, S.W. 1910. Z1. (P). Nematocarcinus ensifer: Williamson, H.C. 1915. NE. (B).

Nematocarcinus ensifer exilis: Williamson, D.I. 1962. LS. (B).

Family Oplophoridae Dana, 1852

Acanthephyra sp.

Acanthephyra var. multispina: Kemp, S.W. 1907. NE. (P). Acanthephyra: Gurney, R. 1942. CG.

Acanthephyra sp.: Barnich, R. 1996. Z3. (P). K

Acanthephyra pelagica (Risso, 1816)

Acanthephyra purpurea: Kemp, S.W. 1907. NE. (P). Acanthephyra purpurea: Williamson, H.C. 1915. NE. (P). Acanthephyra pelagica: Williamson, D.I. 1962. Z8?. (B).

Acanthephyra purpurea Milne Edwards, 1881

Acanthephyra purpurea: Coutière, H. 1906. NE. (P).

Acanthephyra purpurea: Williamson, H.C. 1915. NE. (B). Acanthephyra (?): Gurney, R. 1924a. NE. (P).

* Acanthephyra purpurea: Gurney, R.; Lebour, M.V. 1941.

Z1-Z9.M. (P).

(B).

Acanthephyra purpurea: Gurney, R. 1942. Z2.Z4.Z5.Z8?.Z9?

Acanthephyra purpurea: Kurian, C.V. 1956. Z4-Z9. (P). Acanthephyra purpurea: Williamson, D.I. 1962. Z8?. (B).

Acanthephyra stylorostrata (Bate, 1888)

Acanthephyra stylorostrata: Herring, P.J. 1967. Z1. (L).

Oplophorus spinosus (Brullé, 1939)

? Hoplophorus grimaldii: Gurney, R.; Lebour, M.V. 1941.

Z1-Z5.PL. (P).

Systellaspis sp.

Systellaspis: Williamson, D.I. 1962. CG.

Systellaspis debilis (A. Milne Edwards, 1881)

Larve récemment éclose: Coutière, H. 1906. LS. (P). Acanthephyra debilis: Kemp, S.W. 1910. Z1.Z3.M. (P). Acanthephyra debilis: Williamson, H.C. 1915. NE. (P+B).

* Systellaspis debilis: Gurney, R.; Lebour, M.V. 1941. Z1-

Z3.PL. (L).

Systellaspis debilis: Williamson, D.I. 1962. Z3. (B).

Family Pasiphaeidae Dana, 1852

Pasiphaeidae: Boschi, E.E. 1981. CG.

Pasiphaea multidentata Esmark, 1866

Passiphaea tarda: Björck, W. 1911. Z2 (as Z1). (P).

Pasiphae tarda: Williamson, H.C. 1915. Z2. (as Z1). (B).

? Pasiphaea principalis: Williamson, D.I. 1960b. PS.LS. (P).

Pasiphaea multidentata: Elofsson, R. 1961. Z2-Z4. (P).

Parapasiphae sulcatifrons S.I. Smith, 1884

Pasiphaë sulcatifrons: Kemp, S.W. 1910. NE. (P).

Pasiphaë sulcatifrons: Williamson, H.C. 1915. NE. (P).

Parapasiphae sulcatifrons: Gurney, R. 1942. Z1-Z3.PL?. (B).

Parapasiphae sulcatifrons: Williamson, D.I. 1960b. Z1-Z4.M $(\mathrm{P}+\mathrm{B})$. (B)

Parapasiphae sulcatifrons: Williamson, D.I. 1962. Z1.Z4.M.

Pasiphaea tarda Kröyer, 1865
Pasiphaea tarda: Björck, W. 1911. Z4.M. (P). Pasiphaea tarda: Elofsson, R. 1961. Z1-Z4. (P).

Pasiphaea sivado (Risso, 1816)

Pasiphaea tarda: Stephensen, K. 1916. Z3. (P).

Pasiphaea sivado: Bourdillon Casanova, L. 1960. Z1. (L).

* Pasiphaea sivado: Williamson, D.I. 1960b. Z1.Z2.M. (L+P).

Pasiphaea sivado: Williamson, D.I. 1962. Z1.Z4. (B).

Family Palaemonidae Rafinesque, 1815

Palaemonidae: Webb, G.E. 1921. CG.

Palaemoninae: Gurney, R. 1942. CG.

Unidentified larvae: Lebour, M.V. 1959. NE. (P).

Palaemonidae: Fincham, A.A.; Figueras, A.J. 1986. K.

Palaemoninae: Fincham, A.A.; Figueras, A.J. 1986. CG. K.

Brachycarpus biunguiculatus (Lucas, 1846)

Brachycarpus biunguiculatus: Gurney, R. 1924b. Z3. (P).

Brachycarpus biunguiculatus: Gurney, R. 1938. Z1. (P).

* Brachycarpus biunguiculatus: Gurney, R.; Lebour, M.V.

1941. Z1-Z11.PL. (L+P).

Mesocaris $s p$

Mesocaris sp. II: Barnich, R. 1996. PS. (P). K.

Palaemon $s p$

Palaemon: Barnich, R. 1996. (P+B). K.

Palaemon adspersus Rathke, 1837

Leandri rectirostris: Czerniavsky, V. 1884. Z1. (P).

Leander fabricii: Mortensen, T. 1897. Z1-Z5.PL. (P).

Palaemon fabricii: Williamson, H.C. 1915. Z1-Z5.PL. (P).

Leander adspersus: Sollaud, E. 1923. CG.

Palaemon adspersus: Bourdillon Casanova, L. 1960. Z1. (L).

Palaemon (Palaemon) adspersus: Fincham, A.A.; Williamson,

D.I. $1978 . \mathrm{K}$.

Palaemon adspersus: Makarov, R.R.; Golodetsky, L.A. 1980.

Z1-Z5.PL. (L).

* Palaemon (Palaemon) adspersus: Fincham, A.A. 1985. Z1-

Z6.PL. (L).

Palaemon adspersus: Fincham; A.A.; Figueras, A.J. 1986. Z1-

Z6.PL. (B). K.

Palaemon adspersus: Barnich, R. 1996. Z1-Z6. K.

Palaemon elegans Rathke, 1837

Palaemon squilla: Williamson, H.C. 1915. NE. (B).

Leander squilla: Webb, G.E. 1921. Z1. (P)

Leander squilla: Gurney, R. 1924c. Z1.Z2.Z5. (P).

Leander squilla elegans: Gurney, R. 1927. Z1. (L).

Leander squilla elegans: Gurney, R. 1942. Z4. (B)

Leander squilla typica: Hoglund, H. 1943. Z1-Z6.PL. (L+P).

Palaemon elegans: Tsurnamal, M. 1963. Z1-Z8.PL. (L).

Leander squilla elegans: Wimpenny, R.S.; Titterington, E.

1963. Z1-Z6.PL. (P).

Palaemon elegans: Rochanaburanon, T.; Williamson, D.I.

1976. Z1-Z9.PL. (L).

Palaemon elegans: Carli, A.; Vignola, S. 1977. Z1-Z8.PL. (L),

* Palaemon (Paleander) elegans: Fincham, A.A. 1977. Z1-

Z9.PL. (L).

Palaemon elegans: Carli, A. 1978. Z1-Z8.PL. (B). K.

Palaemon (Paleander) elegans: Fincham, A.A.; Williamson,

D.I. 1978. Z3-Z9.PL. (B). K.

Palaemon elegans: Fincham, A.A.; Figueras, A.J. 1986.

Z1.Z2. (B). K

Palaemon elegans: Barnich, R. 1996. Z1-Z8. K.

Palaemon longirostris H. Milne Edwards, 1837

Leander longirostris: Gurney, R. 1924c. Z1-Z3.Z5.PL. (L+P). Palaemon (Palaemon) longirostris: Fincham, A.A.;

Williamson, D.I. 1978. (B). K.

*Palaemon (Palaemon) longirostris: Fincham, A.A. 1979. Z1-

Z7.PL. (L).

Palaemon longirostris: Fincham, A.A.; Figueras, A.J. 1986.

Z1-Z7.PL. (B). K.

Palaemon longirostris: Barnich, R. 1996. Z1-Z7. (B). K.

Palaemon serratus (Pennant, 1777)

Palaemon serratus: Thompson, J.V. 1836. Z1.Z3.Z5. (L+P). 
Leander serratus: Sollaud, E. 1912. Z1-Z9. (L).

Palaemon serratus: Williamson, H.C. 1915. Z1.Z2.LS. (P).

Leander serratus: Sollaud, E. 1923. Z1-Z9.PL. (L+P).

Leander serratus: Kurian, C.V. 1956. Z2.Z4.Z5. (P).

Palaemon serratus: Sutton, A.H.; Main, G.; Ronald, A. 1969.

Z1. (L).

Palaemon serratus: Carli, A. 1978. Z1-Z8.PL. (B). K.

Palaemon (Palaemon) serratus: Fincham, A.A.; Williamson,

D.I. 1978. (B). K.

* Palaemon (Palaemon) serratus: Fincham, A.A. 1983. Z1-

Z9.PL. (L).

Palaemon serratus: Fincham, A.A.; Figueras, A.J. 1986. Z1-

Z9.PL. (B). K

Palaemon serratus: Yagi, H. 1986. Z1-Z6.PL. (L).

Palaemon serratus: Ramonell, R. 1987. Z1-Z7. (L).

Palaemon serratus: Barnich, R. 1996. Z1-Z8. (P+B). K.

Palaemon xiphias Risso, 1816

Leander xiphias: Heegaard, P. 1963. Z1. (L)

Palaemon xiphias: Carli, A. 1978. Z1-Z7. PL. (B). K.

* Palaemon xiphias: Carli, A.; Marchi, M. 1979. Z1-Z8.PL. (L).

Palaemon xiphias: Barnich, R. 1996. Z1-Z7. (P+B). K.

Palaemonetes varians (Leach, 1814)

Palaemon variabilis: Du Cane, C. 1839. Z1-Z4. (P).

Palaemon varians: Williamson, H.C. 1915. Z1-Z7. (P+B)

Palaemonetes varians: Webb, G.E. 1921. Z4. (P).

Palaemonetes varians: Gurney, R. 1924b. Z1-Z5.PL. (L).

Palaemonetes (Palaemonetes) varians: Fincham, A.A.

Williamson, D.I. 1978. (B). K.

* Palaemonetes (Palaemonetes) varians: Fincham, A.A. 1979.

Z1-Z5.PL1.PL2. (L).

Palaemonetes varians: Fincham, A.A.; Figueras, A.J. 1986.

Z1-Z5.PL. (B). K.

Palaemonetes zariquieyi Sollaud, 1939

* Palaemonetes zariquieyi: Guerao, G. 1993. Z1-Z3.PL. (L).

Periclimenes sp.

Periclimenes sp.: Bourdillon Casanova, L. 1960. Z2-Z8.PL. (P).

Periclimenes sp. EM8: Williamson, D.I. 1967. LS. (P).

Periclimenes sp.: Barnich, R. 1996. Z1-Z8. (P+B). K.

Pontonia flavomaculata Heller, 1864

Pontonia phallusiae: Gourret, P. 1884. Z1. (L).

Pontonia phallusiae: Gourret, P. 1888. Z1. (P+L).

Pontonia flavomaculata: Bourdillon Casanova, L. 1960. Z1. (L).

* Pontonia flavomaculata: Costanzo, G.; Calafiore, N.; Crescenti, N. 1996. Z1-Z8.PL. (L).

Pontonia pinnophylax (Otto, 1821)

Pontonia tyrrehena: Caroli, E. 1926. Z1. (L). (P).

Pontonia pinnophylax: Bourdillon Casanova, L. 1960. Z1-Z3.

Pontonia custos: Heegaard, P. 1963. Z1. (L).

* Pontonia pinnophylax: Calafiore, N.; Costanzo, G.; Gia-

cobbe, S. 1991. Z1-Z8.PL. (L).

Typton spongicola Costa, 1844

Typton spongicola: Lebour, M.V. 1925. Z1. (L).

Typton spongicola: Caroli, E. 1926. Z1. (L).

Typton spongicola: Lebour, M.V. 1949. LS.PL. (P+R)

Typton spongicola: Fincham, A.A.; Williamson, D.I. 1978.

Z1.LS.(B). K.

Family Alpheidae Rafinesque, 1815

Alpheidae: Webb, G.E. 1921. CG.

Alpheidae: Gurney, R. 1942. CG.

Alpheide G.M.1: Bourdillon Casanova, L. 1960. Z1-Z4.Z5?. (P).

Alpheidae: Boschi, E.E. 1981. CG.

Alpheidae Spec. 1: Barnich, R. 1996. Z1-Z5. (P+B).

Alpheus sp.

Alpheus ? dentipes: Pessani, D. 1993. Z1. (P)

Alpheus ? dentipes: Barnich, R. 1996. PL. (P). K.

Alpheus glaber (Olivi, 1792)

Alpheus ruber: Coutière, H. 1907. H. (P).
Alpheus ruber: Williamson, H.C. 1915. NE. (P). Alpheus ruber: Webb, G.E. 1921. Z1-Z9. (P). Alpheus ruber: Stephensen, K. 1923. NE. (P). * Alpheus ruber: Lebour, M.V. 1932a. Z1-Z9. (L). Alpheus ruber: Kurian, C.V. 1956. Z1-Z9. (P). Alpheus glaber: Williamson, D.I. 1967. PS. (P). Alpheus glaber: Williamson, D.I. 1967a. (B). K. Alpheus glaber: Pessani, D. 1993. NE. (P). Alpheus glaber: Barnich, R. 1996. Z1-Z9.PL. (P+B). K.

Alpheus macrocheles (Hailstone, 1835)

* Alpheus macrocheles: Lebour, M.V. 1932a. Z1-Z9. (L). Alpheus macrocheles: Williamson, D.I. 1967a. CG. (B). K. Alpheus macrocheles: Barnich, R. 1996. Z1-Z9.PL. (P). K.

Athanas nitescens (Leach, 1814)

Athanas nitescens: Sars, G.O. 1906. Z1.Z2.LS.PL. (P)

Athanas nitescens: Williamson, H.C. 1915. NE. (P).

Athanas nitescens: Webb, G.E. 1921. Z1-Z3.LS. (P).

Athanas nitescens: Lebour, M.V. 1932a. Z1.Z2. (L).

Athanas nitescens: Gurney, R. 1942. Z3. (B)

Athanas nitescens: Kurian, C.V. 1956. Z2-Z9. (P).

Athanas nitescens: Williamson, D.I. 1967a. CG. K.

Athanas nitescens: Barnich, R. 1996. CG. K.

Synalpheus gambarelloides (Nardo, 1847)

Synalpheus laevimanus: Coutière, H. 1898. Z. (P).

Synalpheus laevimanus: Bourdillon Casanova, L. 1960. Z1-

Z4. (L).

Synalpheus tumidomanus Paulson, 1875

* Synalpheus tumidomanus: Bhuti, G.S.; Shenoy, S.; Sankolli, K.N. 1977. Z1-Z3. (L). ${ }^{1}$

Family Hippolytidae Dana, 1852

Hippolytidae: Webb, G.E. 1921. CG.

Hippolytidae: Boschi, E.E. 1981. CG.

Hippolytidae: Seridji, R. 1989. CG.

Eualus sp.

Eualus: Williamson, D.I. 1957a. K.

Eualus sp.: Barnich, R. 1996. PL. K.

Eualus ? drachi: Barnich, R. 1996. Z1-Z7. (P). K.

Eualus occultus (Lebour, 1936)

Spirontocaris occulta: Lebour, M.V. 1936. Z1.LS. (L+R).

Spirontocaris oculta: Bull, H.O. 1939. (B). K.

Eualus occultus: Williamson, D.I. 1957a. Z1. (B)

Eualus occultus: Bourdillon Casanova, L. 1960. Z1. (L).

* Eualus occultus: Pike, R.B.; Williamson, D.I. 1961a. Z1-

Z9. (P).

Eualus occultus: Barnich, R. 1996. Z1-Z9. (P+B). K.

Hippolyte sp.

Hippolyte: Lebour, M.V. 1931. CG

Hippolyte: Lebour, M.V. 1932. CG.

Hyppolyte: Gurney, R. 1942. CG

Hyppolyte: Williamson, D.I. 1957a. K.

Hippolyte sp.: Barnich, R. 1996. Z1-Z5.PL. (P+B). K.

Hippolyte inermis Leach, 1815

Hippolyte prideauxiana: Lebour, M.V. 1931. Z1-Z3. (L). Hippolyte inermis: Williamson, D.I. 1957a. Z1. (B).

Hippolyte inermis: Bourdillon Casanova, L. 1960. Z1. (L+P).

Hippolyte inermis: Heegaard, P. 1963. Z1. (L).

Hippolyte inermis: Le Roux, A. 1963. Z1-Z4.M. (L).

Hippolyte varians Leach, 1814

* Hippolyte varians: Sars, G.O. 1911. Z1-Z5. PL. (L+P)

Hippolyte varians: Williamson, H.C. 1915. Z1-Z5. (P+B).

Hippolyte varians: Webb, G.E. 1921. Z1. (P).

${ }^{1}$ The taxon recorded in this area is specifically the subspecies $S$ tumidomanus africanus, although its larval description is not available. The larval description of S. tumidomanus (from Indian waters) is included to help identify planktonic larvae of $S$. tumidomanus africanus 
Hippolyte sp.: Webb, G.E. 1921. Z4. (P).

Hippolyte varians: Lebour, M.V. 1931. Z1. (L).

Hippolyte varians: Gurney, R. 1936a. Z1. (P).

Hippolyte varians: Williamson, D.I. 1957a. Z1. (B).

Lysmata $s p$.

Hippolysmata? species R.S.I: Gurney, R. 1937. Z1-Z3. (P).

Lysmata: Gurney, R. 1942. CG.

Lysmata: Williamson, D.I. 1957a. (B). K.

Lysmata spp.: Knowlton, R.E.; Alavi, M.R. 1995. CG.

Lysmata sp.: Barnich, R. 1996. Z1-Z9. (P+B). K.

Lysmata seticaudata (Risso, 1816)

* Miersia clavigera: Caroli, E. 1918. Z1-Z8. (L).

Lysmata ?: Gurney, R. 1924a. D. (P).

Lysmata seticaudata: Kurian, C.V. 1956. Z1.Z2.Z4-Z9. (P).

Lysmata seticaudata: Williamson, D.I. 1957a. LS. (B).

Thoralus sp.

Spirontocaris: Lebour, M.V. 1932. CG.

Thoralus sp.: Williamson, D.I. 1957a. (B). K.

Thoralus sp.: Barnich, R. 1996. Z1-Z9.PL. (P). K.

Thoralus cranchii (Leach, 1817)

Hippolyte cranchii: Williamson, H.C. 1915. Z1. (P).

* Spirontocaris cranchii: Lebour, M.V. 1936. Z1-Z9.PL. (L).

Spirontocaris cranchii: Kurian, C.V. 1956. Z1-Z9. (P).

Thoralus cranchii: Williamson, D.I. 1957a. Z1. (B).

Thoralus cranchii "specimen de marseille": Bourdillon

Casanova, L. 1960. Z1. (L).

Thoralus cranchii "specimen de la manche": Bourdillon

Casanova, L. 1960. Z1. (L).

Thoralus cranchii: Pike, R.B.; Williamson, D.I. 1961a. Z1. (L). Spirontocaris cranchii: Bull, H.O. 1939. K.

Family Processidae Ortmann, 1896

Processidae: Gurney, R. 1942. CG

Processidae: Seridji, R. 1989. CG. K.

Processidae: Barnich, R. 1996a. PS. CG.

Processa sp.

Processa: Lebour, M.V. 1932. CG.

Processa: Barnich, R. 1996. Z1-Z9.PL. (P+B).

Processa sp. 1: Barnich, R. 1996a. PS. (P).

Processa sp. 2: Barnich, R. 1996a. PS. (P).

Processa canaliculata Leach, 1815

$\mathrm{L}+\mathrm{R})$.

Processa canaliculata: Lebour, M.V. 1936a. Z1-Z5.Z8.Z9.PL.

Processa canaliculata: Gurney, R. 1937a. K.

Processa larva allied to P. canaliculata: Lebour, M.V. 1941.

Z1.Z4?.Z5?.Z6-Z8? (B).

Processa canaliculata: Fincham, A.A.; Williamson, D.I. 1978

Z6.CG. (B).

Processa edulis (Risso, 1816)

Processa canaliculata: Gurney, R. 1923. Z1-Z4. (P+R).

Processa edulis: Lebour, M.V. 1936a. Z8. (P).

Processa edulis: Kurian, C.V. 1956. Z1-Z7.LS. (P).

Processa edulis: Fincham, A.A.; Williamson, D.I. 1978. CG.

Processa edulis crassipes: Fincham, A.A.; Williamson, D.I.

1978. Z6. (B). CG.

Processa edulis: Williamson, D.I.; Rochanaburanon, T. 1979.

Processa edulis crassipes: Williamson, D.I.; Rochanabu-

ranon, T. 1979. CG.

Processa edulis edulis: Barnich, R. 1996a. PS. (P).

Processa elegantula Nouvel and Holthuis, 1957

Processa? elegantula: Barnich, R. 1996a. PS. (P).

Processa macrodactyla Holthuis, 1952

Processa macrodactyla: González-Gordillo, J.I.; Rodríguez,

A. 2000. Z1. (L).

Processa modica Williamson, 1979

Processa modica: Fincham, A.A.; Williamson, D.I. 1978. CG. (B).

Processa modica modica: Fincham, A.A.; Williamson, D.I.
1978. Z5. (B)

* Processa modica modica: Williamson, D.I.; Rochanabura-

non, T. 1979. Z1-Z7.LS. (P)

Processa modica carolii: Barnich, R. 1996a. Z8. (P).

Processa nouveli Al-Adhub and Williamson, 1975

Processa canaliculata: Gurney, R. 1923. Z5-Z9. (P+R)

Processa canaliculata: Kurian, C.V. 1956. Z1.Z5.Z6.LS. (P).

Processa nouveli: Fincham, A.A.; Williamson, D.I. 1978. CG Processa nouveli holthuisi: Fincham, A.A.; Williamson, D.I.

1978. Z1.Z6. (B)

*Processa nouveli holthuisi: Williamson, D.I.; Rochanaburanon, T. 1979. Z1-Z9. (L).

Processa nouveli nouveli: Barnich, R. 1996a. PS. (P).

Familia Pandalidae Haworth, 1825

Icotopus arcurostris: Bate, C.S. 1888. NE. (P).

Pandalidae: Webb, G.E. 1921. CG.

Pandalidae: Lebour, M.V. 1940. CG.

Pandalidae: Gurney, R. 1942. CG. K.

Pandalidae: Boschi, E.E. 1981. CG.

Pandalidae spec. 1: Barnich, R. 1996. Z1. (P). K.

Chlorotocus crassicornis (Costa, 1871)

Chlorotocus crassicornis: Heegaard, P. 1969. Z1. (L).

Dichelopandalus bonnieri (Caullery, 1896)

Dichelopandalus bonnieri: Pike, R.B.; Williamson, D.I. 1964.

Z1-Z6. (L+R)

Pandalus (Dichelopandalus) bonnieri: Lebour, M.V. 1940.

Z1-Z5. (L+P).

Pandalina sp.

Pandalina: Lebour, M.V. 1932. CG.

Pandalina: Lebour, M.V. 1940. CG.

Pandalina brevirostris (Rathke, 1843)

Pandalus (Pandalina) brevirostris: Sars, G.O. 1900. Z5 (as

Z6). (P).

Pandalus brevirostris: Williamson, H.C. 1915. Z6. (B).

Pandalus brevirostris: Webb, G.E. 1921. Z4. (P).

Pandalina brevirostris: Gurney, R. 1926. Z1. (L).

Pandalina brevirostris: Lebour, M.V. 1940. Z1. (L).

Pandalina brevirostris: Gurney, R. 1942. Z1. K.

Pandalina brevirostris: Williamson, D.I. 1957. Z4. (P).

(P). Pandalina brevirostris: Bourdillon Casanova, L. 1960. LS.

* Pandalina brevirostris: Pike, R.B.; Williamson, D.I. 1964

Z1-Z7. (L+R)

Pandalina brevirostris: Barnich, R. 1996. Z1-Z7. (P+K).

Plesionika sp.

Plesionika sp.: Bourdillon Casanova, L. 1960. Z4. (P).

Plesionika sp.: Barnich, R. 1996. Z1-Z11. (P+B). K.

Plesionika acanthonotus (S.I. Smith, 1882)

Plesionika acanthonotus: Bourdillon Casanova, L. 1960. Z1. (L).

Plesionika edwardsii (Brandt, 1851)

Plesionika edwardsii: Mura, M.; Pessani, D. 1994. Z1. (L).

Plesionika martia (A. Milne Edwards, 1883)

Plesionika martia?: Williamson, D.I. 1967a. K.

Stylopandalus richardi (Coutière, 1905)

* Parapandalus richardi: Lebour, M.V. 1940. Z1-Z9.PL.

$(\mathrm{L}+\mathrm{P})$.

Parapandalus richardi: Gurney, R. 1942. K.

Family Crangonidae Haworth, 1825

Crangon: Claus, C. 1861. Z. (P).

Crangonidae: Webb, G.E. 1921. CG.

Crangonidae: Lebour, M.V. 1931. CG.

Crangonidae: Gurney, R. 1942. CG.

Crangon dalli: Birshteyn, A.A. 1938. LS?. (P).

Crangonidae: Barnich, R. 1996. PL. K.

Aegaeon lacazei (Gourret, 1887) 
Pontocaris lacazei: De Simon, M. 1979. Z1.Z4. (L). Pontophilus sp.: Williamson, D.I. 1960. K.

Aegaeon cataphractus (Olivi, 1792)

Pontocaris cataphracta: Bourdillon Casanova, L. 1960.

Z1.Z2. $(\mathrm{L}+\mathrm{P}+\mathrm{B})$.

Pontocaris cataphractus: Williamson, D.I. 1960. Z4?. (B).

Pontocaris cataphracta: Seridji, R. 1971. Z2. (P).

Crangon sp.

Crangon: Lebour, M.V. 1931. K.

Crangon crangon (Linnaeus, 1758)

Crangon vulgaris: Sars, G.O. 1890. Z1.PL. (P)

Crangon vulgaris: Gurney, R. 1903. K.

Crangon vulgaris: Williamson, H.C. 1915. Z1-Z5. (P).

Crangon vulgaris: Webb, G.E. 1921. Z4. (P).

Crangon vulgaris: Lebour, M.V. 1931. Z1. (L). K.

Crangon crangon: Williamson, D.I. 1960. Z1. (B)

Crangon crangon: Bourdillon Casanova, L. 1960. Z1-Z5.

$(\mathrm{P}+\mathrm{B}) . \mathrm{K}$.

* Crangon crangon: Gurney, A.R. 1982. Z1-Z6.PL. (L).

Philocheras sp.

Crangon larvae: Claus, C. 1884. Z.Y. (P)

Philocheras: Lebour, M.V. 1931. CG. K.

Philocheras: Williamson, D.I. 1960. K.

Philocheras: Barnich, R. 1996. Z1-Z5.PL. (P).

Philocheras bispinosus

Philocheras bispinosus: Lebour, M.V. 1931. Z1. (L). K.

Philocheras bispinosus: Kurian, C.V. 1956. Z3-Z5. (P).

Philocheras bispinosus: Bourdillon Casanova, L. 1960. K.

Philocheras bispinosus bispinosus (Hailstone, 1835)

Cheraphilus nanus: Sars, G.O. 1890. Z5.PL. (P).

Cheraphilus nanus: Gurney, R. 1903. Z1. (B). K.

Crangon nanus: Williamson, H.C. 1915. Z1.Z5.PL. (B).

(B). K.

Philocheras bispinosus bispinosus: Williamson, D.I. 1960. Z5.

Philocheras bispinosus bispinosus: Pike, R.B.; Williamson,

D.I. 1961. Z1-Z5. (P).

Philocheras bispinosus bispinosus: Pessani, D.; Godino, C.

1991. CG.

Philocheras bispinosus bispinosus: Barnich, R. 1996. CG.

Philocheras bispinosus neglectus (Sars, 1883)

Philocheras bispinosus neglectus: Williamson, D.I. 1960. Z5.

(B). K.

* Philocheras bispinosus neglectus: Pike, R.B.; Williamson,

D.I. 1961. Z1-Z5. (L).

Philocheras bispinosus neglectus: Pessani, D.; Godino, C.

1991. CG.

Philocheras bispinosus neglectus: Barnich, R. 1996. CG.

Philocheras echinulatus (Sars, 1861)

Cheraphilus echinulatus: Sars, G.O. 1890. Z1.Z5. (P).

Cheraphilus echinulatus: Gurney, R. 1903. K.

Crangon echinulatus: Williamson, H.C. 1915. Z1. (B)

Philocheras echinulatus: Bourdillon Casanova, L. 1960. K.

Philocheras echinulatus: Williamson, D.I. 1960. Z1. (B). K.

Philocheras echinulatus: Barnich, R. 1996. CG.

Philocheras fasciatus (Risso, 1816)

Aegeon fasciatus: Gurney, R. 1903. Z1. (L). K

Crangon fasciatus: Gurney, R. 1903a. Z1.LS. (L+P).

Crangon fasciatus: Williamson, H.C. 1915. Z1. (B).

Aegeon fasciatus: Webb, G.E. 1921. Z1.Z4. (P).

Philocheras fasciatus: Lebour, M.V. 1931. Z2. (P+B). K.

Philocheras fasciatus: Bourdillon Casanova, L. 1960. K.

Philocheras fasciatus: Williamson, D.I. 1960. Z1. (B). K.

Philocheras fasciatus: Pessani, D.; Godino, C. 1991. CG.

Philocheras fasciatus: Barnich, R. 1996. CG.

* Philocheras fasciatus: González-Gordillo, J.I.; Rodríguez,

A. 2000. Z1-Z4. (L).

Philocheras monacanthus (Hothuis, 1961)

* Philocheras monacanthus: González-Gordillo, J.I.; Santos,
A. dos; Rodríguez, A. 2000. Z1-Z5. (L). K.

Philocheras sculptus (Bell, 1847)

Philocheras sculptus: Lebour, M.V. 1931. Z1. (L). K. Philocheras sculptus: Kurian, C.V. 1956. Z1.Z5. (P).

Philocheras sculptus: Bourdillon Casanova, L. 1960. K. Philocheras sculptus: Williamson, D.I. 1960. Z1. (P+B). K. Philocheras sculptus: Seridji, R. 1971. Z3. (P).

Philocheras sculptus: Pessani, D.; Godino, C. 1991. CG.

Philocheras sculptus: Barnich, R. 1996. CG. K.

Philocheras trispinosus (Hailstone, 1835)

Cheraphilus trispinosus: Gurney, R. 1903. Z1. (L). K.

Crangon trispinosus: Gurney, R. 1903a. Z1.Z4.LS.PL. (L).

Crangon trispinosus: Williamson, H.C. 1915. Z1. (P).

Philocheras trispinosus: Lebour, M.V. 1931. Z1.Z5. (L). K

Philocheras trispinosus: Bourdillon Casanova, L. 1960. K.

Philocheras trispinosus: Williamson, D.I. 1960. Z1.Z5.

( $\mathrm{L}+\mathrm{B})$. K.

Philocheras trispinosus: Pike, R.B.; Williamson, D.I. 1961.

Z5. (P).

*Philocheras trispinosus: Pessani, D.; Godino, C. 1991. Z1-

Z5. (L).

Philocheras trispinosus: Barnich, R. 1996. CG.

Pontophilus norvegicus (Sars, 1861)

Pontophilus norvegicus: Sars, G.O. 1890. Z2.Z5.M. (P).

Pontophilus norvegicus: Gurney, R. 1903. K.

Crangon norvegicus: Williamson, H.C. 1915. Z2.M. (P).

Pontophilus norvegicus: Kurian, C.V. 1956. Z1. (P).

Pontophilus norvegicus: Bourdillon Casanova, L. 1960. K.

Pontophilus norvegicus: Williamson, D.I. 1960. Z5. (B).

Pontophilus norvegicus: Barnich, R. 1996. CG.

Pontophilus spinosus (Leach, 1815)

Pontophilus spinosus: Sars, G.O. 1890. Z1.Z5. (P).

Pontophilus spinosus: Gurney, R. 1903. K.

Crangon spinosus: Williamson, H.C. 1915. Z1. (P).

Pontophilus spinosus: Webb, G.E. 1921. Z1.Z4. (P)

Pontophilus spinosus: Gurney, R. 1942. Z1. (B).

Pontophilus spinosus: Kurian, C.V. 1956. Z2. (P).

Pontophilus spinosus: Bourdillon Casanova, L. 1960. Z1. (P). K.

Pontophilus spinosus: Williamson, D.I. 1960. Z1.Z5. (B).

Pontophilus spinosus: Barnich, R. 1996. Z1-Z5. (P+B). K.

Family Glyphocrangonidae S.I. Smith, 1884

Glyphocrangonidae: Boschi, E.E. 1981. CG.

Infraorder ASTACIDEA Latreille, 1803

Family Nephropidae Dana, 1852

Homarus gammarus (Linnaeus, 1758)

(P).

Homarus gammarus: Nichols, J.H.; Lawton, P. 1978. Z1-Z4.

* Homarus vulgaris: Sars, G.O. 1875. Z1-Z3. (L).

Homarus vulgaris: Chadwick, H.C. 1905. Z1-Z3. (L).

Astacus gammarus: Williamson, H.C. 1915. Z1-Z3.PL. (P).

Homarus vulgaris: Webb, G.E. 1921. Z1-Z3. (L+B).

Homarus gammarus: Thiriot, A. 1974. NE. (B).

Homarus gammarus: Williamson, D.I. 1983. Z3. (B).

Homarus gammarus: Ingle, R.W. 1992. CG.

Nephrops norvegicus (Linnaeus, 1758)

Nephrops norvegicus: Sars, G.O. 1884. Z1.Z2.M. (P).

Astacus norvegicus: Williamson, H.C. 1915. Z2.LS.(B)

Nephrops norvegicus: Jorgensen, O.M. 1925a. Z1-Z3.PL. (P).

Nephrops norvegicus: Santucci, R. 1926. Z1-Z3.PL (P).

Nephrops norvegicus: Santucci, R. 1927. Z1-Z3.PL. (P).

Nephrops norvegicus: Gurney, R. 1942. Z1-Z3. (B).

Nephrops norvegicus: Kurian, C.V. 1956. Z1. (P).

Nephrops norvegicus: Andersen, F.S. 1962. Z1-Z3. (P).

Nephrops norvegicus: Williamson, D.I. 1983. Z3. (B).

Nephrops norvegicus: Ingle, R.W. 1992. CG.

Infraorder THALASSINIDEA Latreille, 1825

Thalassinidea: Barnich, R. 1996. PL. (K). 
Family Axiidae Huxley, 1879

Axius stirhynchus Leach, 1815 Axius stirhynchus: Williamson, H.C. 1915. Z1.Z2. (P+B). Axius stirhynchus: Webb, G.E. 1921. Z1.Z2.PL (P+R).

Axiidae. Species I: Gurney, R. 1924a. Z1. (P). Axius stirhynchus: Gurney, R. 1942. Z1.Z2. (B). Axius stirhynchus: Kurian, C.V. 1956. Z1?. (P). Axius stirhynchus: Bourdillon Casanova, L. 1960. Z1.Z2. (P). Axius stirhynchus: Thiriot, A. 1974. NE. (B).

Axius stirhynchus: Ingle, R.W. 1992. CG.

Axius stirhynchus: Barnich, R. 1996. Z1.Z2.PL. (P+K).

Calocarides coronatus (Trybom, 1904) Calocarides coronatus: Elofsson, R. 1959. NE. (P).

Family Calocarididae Ortmann, 1891

Calocaris macandreae Bell, 1846 Calocaris macadreae: Sars, G.O. 1884. Z2-Z3 (not Z1). (P). Calocaris macandreae: Björck, W. 1913. Z3.PL. (P). Calocaris macandreae: Williamson, H.C. 1915. Z1.Z2.PL. $(\mathrm{P}+\mathrm{B})$.

Calocaris macandreae: Bull, H.O. 1933. Z1.Z2. (L). Calocaris macandreae: Gurney, R. 1942. Z1-Z3. (P+B). Calocaris macandreae: Bourdillon Casanova, L. 1960.

ZA.ZB.PL. (L+P).

Calocaris macandreae: Thiriot, A. 1974. Z1-Z3. (B).

Calocaris macandreae: Ingle, R.W. 1992. CG. (B).

Calocaris macandreae: Barnich, R. 1996. Z1-Z3.PL. (B). K.

Family Callianassidae Dana, 1852

Hippolyte: Claus, C. 1861. Z. (P).

Callianassinae: Boschii, E.E. 1981. CG.

Callianassidae: Barnich, R. 1996. PL. K.

? Callianassa sp. type I: Barnich, R. 1996. Z1-Z5. (P). K.

? Callianassa sp. type II: Barnich, R. 1996. Z1.Z2. (P). K.

Callianassa sp.

Axius Stirynchus: Cano, G. 1891a. Z1-Z4. (P).

Callianassa subterranea (Montagu, 1808)

Callianassa subterranea: Mayer, P. 1877. NL.

Calocaris macandreaea: Sars, G.O. 1884. Z1. (P).

Callianassa subterranea: Williamson, H.C. 1915. Z1.Z2.Y. (P).

Callianassa subterranea: Boraschi, L. 1921. Z1. (P).

Callianassa subterranea: Webb, G.E. 1921. Z1.Z4.PL. (P+R).

* Callianassa subterranea: Lutze, J. 1938. Z1-Z4.M. (L).

Callianassa subterranea: Gurney, R. 1942. Z1-Z5. (P+B).

Callianassa subterranea: Kurian, C.V. 1956. Z1-Z3.Z5. (P).

Callianassa subterranea: Seridji, R. 1989. CG.

Callianassa subterranea: Ingle, R.W. 1992. Z5. (B).

Callianassa tyrrhena (Petagna, 1792)

Callianassa subterranea: Claus, C. 1876. Z. (P).

Callianassa (subterranea): Claus, C. 1885. Y. (P).

Callianassa subterranea: Cano, G. 1891a. Z1-Z3.PL. (L).

Callianassa stebbingi: Heegaard, P. 1963. Z1. (L).

Family Laomediidae Borradaile, 1903

Jaxea $s p$.

Jaxea: Claus, C. 1884. Z. (P).

Jaxea nocturna Nardo, 1847

Jaxea nocturna: Claus, C. 1884. Z1.Z3. (P)

Trachelifer: Brooks, 1889. Z2.Z4. (P).

Calliaxis adriatica: Cano, G. 1891a. Z1-Z3. (L).

Jaxea nocturna: Bouvier, E.L. 1914. Z1-Z4.Y1-Y3. (P+B)

Taxea nocturna: Williamson, H.C. 1915. Z1. (B).

Jaxea nocturna: Boraschi, L. 1921. Z1.Z2. (P).

* Jaxea nocturna: Caroli, E. 1924. Z1-Z6.PL. (P).

Jaxea nocturna: Tattersall, W.M. 1938. L5.PL. (P).

Jaxea nocturna: Gurney, R. 1942. CG.

Jaxea nocturna: Kurian, C.V. 1956. Z2-Z4. (P).

Jaxea nocturna: Seridji, R. 1989. CG.

Jaxea nocturna: Ingle, R.W. 1992. CG.

Jaxea nocturna: Barnich, R. 1996. Z1-Z6.PL. (P). K.
Family Upogebiidae Borradaile, 1903

Upogebiidae: Barnich, R. 1996. PL. K.

Upogebia sp.

Gebia littoralis: Cano, G. 1891a. Z1-Z4.PL. (P).

Upogebia sp.: Webb, G.E. 1921. CG.

Upogebia sp. B: Bourdillon Casanova, L. 1960. NE. (P)

Upogebia pusilla ?: Bourdillon Casanova, L. 1960. Z1-Z4. (P).

Upogebia: Thiriot, A. 1974. CG

Upogebia: Boschi, E.E. 1981. CG.

Upogebia sp.: Barnich, R. 1996. Z1-.Z4.PL. (P). K.

Upogebia deltaura (Leach, 1815)

Gebia littoralis: Sars, G.O. 1884. Z1-Z3. (P).

* Upogebia deltura: Webb, G.E. 1919. Z1-Z4.PL. (P+R).

Upogebia deltaura: Gurney, R. 1942. CG.

Upogebia deltaura: Kurian, C.V. 1956. Z2-Z4. (P).

Upogebia deltaura: Bourdillon Casanova, L. 1960. Z1-Z4. (B).

Upogebia deltaura: Heegaard, P. 1963. Z1. (L).

Upogebia deltaura: Seridji, R. 1989. CG.

Upogebia deltaura: Ingle, R.W. 1992. Z3. (B).

Upogebia pusilla (Petagna, 1792)

Gebia littoralis: Mayer, P. 1877. NL.

Gebia littoralis: Williamson, H.C. 1915. Z1.Z2.LS. (B).

Gebia littoralis: Webb, G.E. 1919. CG.

Upogebia littoralis: Dolgolskaya, M.A. 1954. Z1-Z4.M.

Upogebia littoralis: Heegaard, P. 1963. Z1. (L).

* Upogebia pusilla: Dolgopolskaya, M.A. 1969. Z1-Z4.M.

Upogebia pusilla: Seridji, R. 1989. CG.

\section{Infraorder PALINURA Latreille, 1902}

Family Polychelidae Wood Mason, 1875

Eryoneicus caecus: Bate, S.C. 1888. Q. (P).

Eryoneicus sp. juv.: Selbie, C.M. 1914. NE. (P).

Polychelidae: Ingle, R.W. 1992. CG.

Eryoneicus puritanii Lo Bianco, 1903

Eryoneicus puritanii: Lo Bianco, S. 1903. Q. (P).

Eryoneicus puritanii: Williamson, D.I. 1983. M. (B). K.

Eryoneicus spinoculatus Bouvier, 1905

Eryoneicus spinoculatus: Bernard, F. 1953. NE. K.

Eryoneicus spinoculatus: Williamson, D.I. 1983. M. (B). K.

Polycheles sp. Polycheles sp.: Balss, H. 1925. Z1. (P).

Eryoneicus sp.: Gurney, R. 1942. CG.

Polycheles typhlops Heller, 1862

Polycheles typhlops: Guerao, G.; Abelló, P. 1996. Z1. (L).

Family Palinuridae Latreille, 1802

Palinuridae: Gurney, R. 1942. CG.

Palinuridae: Seridji, R. 1989. CG.

Palinurus sp.

Palinurus: Ingle, R.W. 1992. CG.

Palinurus sp.: Barnich, R. 1996. Z1.Z2. (P+B). K.

Palinurus elephas (Fabricius, 1787)

Palinurus vulgaris: Cunningham, J.T. 1891. P1.P2. (L+P).

Palinurus vulgaris: Bouvier, E.L. 1913. U. (P).

Palinurus vulgaris: Bouvier, E.L. 1913a. U. (P).

Palinurus vulgaris: Bouvier, E.L. 1914a. P1-P7.P9.P10.U. (P).

Palinurus vulgaris: Williamson, H.C. 1915. P1.P3. (B).

Palinurus vulgaris: Webb, G.E. 1921. NE. (B).

* Palinurus vulgaris: Santucci, R. 1925. P1-P9. (P).

Palinurus vulgaris: Santucci, R. 1926b. U.PL. (P).

Palinurus vulgaris: Gurney, R. 1942. Z1-Z3. (B).

Palinurus vulgaris: Caroli, E. 1946. U. (P)

Palinurus vulgaris: Kurian, C.V. 1956. P1. (P).

Palinurus elephas: Williamson, D.I. 1983. P3.U. (B).

Palinurus elephas: Ingle, R.W. 1992. Z3. (B).

Family Scyllaridae Latreille, 1825

Scyllaridae: Gurney, R. 1942. CG. 
Scyllaridae: Seridji, R. 1989. CG.

Scyllaridae: Ingle, R.W. 1992. CG.

Scyllarides $s p$.

Scyllarides: Boschi, E.E. 1981. CG.

Scyllarides: Barnich, R. 1996. Z1.Z2. (B).

Scyllarides latus (Latreille, 1803)

Pseudibacus veranyi: Bouvier, E.L. 1913. NE. (P).

? Scyllarides latus: Stephensen, K. 1923. NE. (P).

? Thenus orientalis: Stephensen, K. 1923. NE. (P).

Scyllarus latus: Santucci, R. 1925a. P1. (P).

Thenus orientalis ?: Santucci, R. 1926a. P1. (P).

Scyllarides latus: Santucci, R. 1928. P1. (P).

Scyllarus sp.

Scyllarus: Gurney, R. 1936. CG.

Scyllarus sp.: Boschi, E.E. 1981. CG.

Scyllarus: Barnich, R. 1996. Z1.Z2. (P+B). K.

Scyllarus arctus (Linnaeus, 1758)

Phyllosoma mediterranea: Claus, C. 1863. P1.P8. (P).

Scyllarus arctus: Williamson, H.C. 1915. NE. (B).

Scyllarus arctus: Stephensen, K. 1923. P1-P9.N. (P)

* Scyllarus arctus: Santucci, R. 1925a. P1-P9. (P).

Scyllarus arctus: Fedele, M. 1926. LS. (P).

Scyllarus arctus: Gurney, R. 1942. Z1.LS. (B).

Scyllarus arctus: Kurian, C.V. 1956. P1-P8. (P).

Scyllarus arctus: Williamson, D.I. 1983. P1.U. (B)

Scyllarus posteli Forest, 1963

Scyllarus posteli: González-Gordillo, J.I.; Rodríguez, A. 2000. Z1. (L).

Scyllarus pygmaeus (Bate, 1888)

Scyllarus pygmaeus: Mura, M.; Pessani, D. 1994. Z1. (L).

Infraorder ANOMURA MacLeay, 1838

Anomura: Pike, R.B.; Williamson, D.I. 1960. CG.

Anomura: Boschi, E.E. 1981. CG.

Family Diogenidae Ortmann, 1892

Diogenidae: Bourdillon Casanova, L. 1960. CG.

Diogenidae: Pike, R.B.; Williamson, D.I. 1960. CG.

Diogenidae: Seridji, R. 1989. CG.

Diogenidae: Ingle, R.W. 1992. CG.

Diogenidae: Barnich, R. 1996. PL. K.

Calcinus sp

Larva D (Pagurus calidus ?): Boraschi, L. 1921. Z1. (P).

Calcinus tubularis (Linnaeus, 1767)

Glaucothoe grimaldii: Bouvier, E.L. 1922. M. (P).

Calcinus ornatus: Bourdillon Casanova, L. 1960. Z1. (P). K

* Calcinus ornatus: Pike, R.B.; Williamson, D.I. 1960. Z1-

Z5.M. (L+P). K.

Calcinus ornatus: Thiriot, A. 1974. CG.

Calcinus tubularis: Barnich, R. 1996. Z1-Z5. (P+B). K.

Calcinus ornatus: Barnich, R. 1996. PL. K.

Clibanarius erythropus (Latreille, 1818)

Pagure misanthrope: Hesse, M. 1876. Z1. (P).

Zoea Virbii?: Czerniavsky, V. 1884. Z1. (P).

Clibanarius misanthropus: Issel, R. 1910. Z1. (L).

Pagurus misanthropus: Williamson, H.C. 1915. Z1. (P+B).

Clibanarius: Boraschi, L. 1921. Z1. (P).

Clibanarius misanthropus: Carayon, J. 1942. M. (P).

Clibanarius erythropus: MacDonald, J.D.; Pike, R.B.;

Williamson, D.I. 1957. (B). K.

Clibanarius misanthropus: Dechancé, M. 1958. Z1-Z4.M. (P) Clibanarius erythropus: Dechancé, M.; Forest, J. 1958. G. (R). Clibanarius erythropus: Pike, R.B.; Williamson, D.I. 1958.

Z1.M. (B)

Clibanarius erythropus: Bourdillon Casanova, L. 1960. Z1.

(P). K.

Clibanarius erythropus: Pike, R.B.; Williamson, D.I. 1960.

$\mathrm{Z} 1-\mathrm{Z} 3$. $(\mathrm{L}+\mathrm{P})$. K.

Clibanarius erythropus: Le Roux, A. 1966a. Z1-Z4.M. (L).
Clibanarius erythropus: Thiriot, A. 1974. CG.

Clibanarius erythropus: Seridji, R. 1989. Z4. (P).

Clibanarius erythropus: Barnich, R. 1996. Z1-Z5.PL. (P+B). K.

Dardanus sp.

Dardanus: Dechancé, M. 1962. CG.

Dardanus: Boschi, E.E. 1981. CG.

Dardanus ? calidus: Seridji, R. 1989. Z3. (P).

Dardanus arrosor (Herbst, 1796)

Pagurus arrosor: Issel, R. 1910. Z1. (L).

Pagurus arrosor: Boraschi, L. 1921. Z1. (P).

Dardanus arrosor: Bourdillon Casanova, L. 1960. Z1. (P). K.

Dardanus arrosor: Pike, R.B.; Williamson, D.I. 1960. Z1. (P). K.

Dardanus arrosor: Dechancé, M. 1962. Z1.Z2.

Dardanus arrosor: Thiriot, A. 1974. CG.

* Dardanus arrosor: Kurata, H. 1968. Z1-Z7.M. (L).

Dardanus arrosor: Boschi, E.E. 1981. Z1.M. (B).

Dardanus arrosor: Barnich, R. 1996. Z1. (P). K.

Dardanus calidus (Risso, 1826)

Glaucothoe rostrata: Miers, E.J. 1881. G. (P)

Diogenes pugilator (Roux, 1829)

Diogenes pugilator: Gurney, R. 1927. Z1-Z4.G. (P).

Diogenes pugilator: Menon, M.K. 1937. Z1-Z4.M. (P).

Diogenes pugilator: Gurney, R. 1942. K.

* Diogenes pugilator: MacDonald, J.D.; Pike, R.B.;

Williamson, D.I. 1957. Z1-Z5.M. (L+P+R). K. Diogenes pugilator: Pike, R.B.; Williamson, D.I. 1958. Z1-

Z4.M. (B).

Diogenes pugilator: Bourdillon Casanova, L. 1960. Z1. (P). K. Diogenes pugilator: Pike, R.B.; Williamson, D.I. 1960. K. Diogenes pugilator: Thiriot, A. 1974. CG.

Diogenes pugilator: Barnich, R. 1996. Z1-Z5.PL. (P+B). K.

Paguristes sp.

Paguristes: Boschi, E.E. 1981. CG.

Paguristes eremita (Linnaeus, 1767)

Paguristes oculatus: Issel, R. 1910. Z3.G. (L).

Paguristes oculatus: Gurney, R. 1942. K.

Paguristes oculatus: Bourdillon Casanova, L. 1960. Z1.Z2.G.

(P). K.

* Paguristes oculatus: Pike, R.B.; Williamson, D.I. 1960. Z1-

Z3.M. K.

Paguristes oculatus: Thiriot, A. 1974. CG.

Paguristes oculatus: Seridji, R. 1989. Z1-Z3. (B).

Paguristes oculatus: Barnich, R. 1996. PL. K.

Family Paguridae Latreille, 1803

Paguridae: Webb, G.E. 1921. CG

Paguridae: Gurney, R. 1942. CG.

Paguroidea: MacDonald, J.D.; Pike, R.B.; Williamson, D.I.

1957. CG.

Paguridae: Pike, R.B.; Williamson, D.I. 1958. (B). K.

Paguridae: Bourdillon Casanova, L. 1960. CG.

Paguridae: Pike, R.B.; Williamson, D.I. 1960. CG.

Paguridae: Rice, A.L.; Williamson, D.I. 1977. Z1.Z4. (P).

Paguridae: Boschi, E.E. 1981. CG.

Paguridae: Seridji, R. 1989. CG.

Paguridae: Ingle, R.W. 1992. CG

Paguridae: Barnich, R. 1996. PL. K.

Anapagurus sp.

Anapagurus sp.: MacDonald, J.D.; Pike, R.B.; Williamson,

D.I. 1957. CG.

Anapagurus: Barnich, R. 1996. CG. (P+B).

Anapagurus bicorniger A. Milne Edwards and Bouvier, 1892 * Anapagurus bicorniger: Pike, R.B.; Williamson, D.I. 1960.

Z1-Z4.M. (L+P+R).

Anapagurus bicorniger: Dechancé, M.; Forest, J. 1962. G. (P).

Anapagurus bicorniger: Barnich, R. 1996. Z1-Z4.PL. (B+P). K.

Anapagurus breviaculeatus Fenizia, 1937

Anapagurus laevis: Pike, R.B.; Williamson, D.I. 1958. Z1-

Z4.M. (B). K. 
Anapagurus breviaculeatus: Barnich, R. 1996. Z1-Z4.PL. (B). K.

Anapagurus chiroacanthus (Lilljeborg, 1856)

Spiropagurus chiroacanthus: Sars, G.O. 1889. Z1.Z4.M. (P).

Pagurus chiroacanthus: Williamson, H.C. 1915. Z1.LS.

$(\mathrm{P}+\mathrm{B})$.

Anapagurus chiroacanthus: Gurney, R. 1942. K.

Anapagurus chiroacanthus: Kurian, C.V. 1956. Z1-Z4. (P).

* Anapagurus chiroacanthus: MacDonald, J.D.; Pike, R.B.;

Williamson, D.I. 1957. Z1-Z4.G. (L+P+R). K.

Anapagurus chiroacanthus: Pike, R.B.; Williamson, D.I.

1958. Z1-Z4.M. (B). K.

Anapagurus chiroacanthus: Pike, R.B.; Williamson, D.I.

1960. Z1.K.

* Anapagurus chiroacanthus: Ingle, R.W. 1990. Z1-Z4.M. (L). Anapagurus chiroacanthus: Ingle, R.W. 1992. Z1-Z4. (B).

Anapagurus chiroacanthus: Barnich, R. 1996. Z1-Z4.PL.

$(\mathrm{P}+\mathrm{B}) . \mathrm{K}$

Anapagurus hyndmanni (Bell, 1845)

Anapagurus hyndmanni: Gurney, R. 1942. Z1.M. (B).

* Anapagurus hyndmanni: MacDonald, J.D.; Pike, R.B.;

Williamson, D.I. 1957. Z1-Z4.G. (L+P+R). K.

Anapagurus hyndmanni: Pike, R.B.; Williamson, D.I. 1958.

Z1-Z4.M. (B). K.

Anapagurus laevis (Bell, 1846)

* Anapagurus laevis: MacDonald, J.D.; Pike, R.B.;

Williamson, D.I. 1957. Z1-Z4.G. (L+P+R). K.

Anapagurus laevis: Pike, R.B.; Williamson, D.I. 1958. Z1-

Z4.M. (B). K.

Anapagurus laevis: Pike, R.B.; Williamson, D.I. 1960. K.

Anapagurus laevis: Barnich, R. 1996. Z1-Z4.PL. (P+B). K.

Anapagurus petiti Dechancé and Forest, 1962

Anapagurus sp. N1: Pike, R.B.; Williamson, D.I. 1960. Z4.M. (P)

Anapagurus petiti: Dechancé, M.; Forest, J. 1962. G. (P).

Anapagurus petiti: Barnich, R. 1996. Z3.Z4.PL. (P+B). K.

Cestopagurus timidus (Roux, 1830)

Catapaguroides timidus: Boraschi, L. 1921. Z1. (P).

Catapaguroides timidus: Dechancé, M.; Forest, J. 1958. G. (R).

Catapaguroides timidus: Pike, R.B.; Williamson, D.I. 1958.

Z1-Z4. (B). K.

* Catapaguroides timidus: Pike, R.B.; Williamson, D.I. 1960.

Z1-Z4.M. (P+R). K.

Catapaguroides timidus: Dechancé, M. 1961. Z1-Z4. (P).

Cestopagurus timidus: Barnich, R. 1996. Z1.Z4.PL. (P+B). K

Nematopagurus longicornis A. Milne Edwards and Bouvier, 1892 Latreillia ?: Cano, G. 1893. LS. (P).

Catapaguroides timidus: Issel, R. 1910. Z1. (L).

Anapagurus sp. N3: Pike, R.B.; Williamson, D.I. 1960. Z1-

Z4. $(\mathrm{L}+\mathrm{P}+\mathrm{R})$.

Nematopagurus longicornis: Barnich, R. 1996. Z1-Z4. (P+B). K.

Pagurus sp.

Pagurus: Claus, C. 1885. Z4. (P).

Pagurus: Boschi, E.E. 1981. CG.

Pagurus: Barnich, R. 1996. CG.

Pagurus alatus (Fabricius, 1775)

Eupagurus excavatus: Issel, R. 1910. Z1. (P).

Pagurus alatus: Pike, R.B.; Williamson, D.I. 1958. Z1.Z3.Z4.

(B). K

Pagurus variabilis: Samuelsen, T.J. 1972a. Z1-Z3. (L).

Pagurus alatus: Barnich, R. 1996. Z1-Z4.PL. (B). K.

Pagurus anachoretus Risso, 1826

* Pagurus anachoretus: Pike, R.B.; Williamson, D.I. 1960

Z1-Z4.M. (L+R). K.

Pagurus anachoretus: Barnich, R. 1996. Z1-Z4.PL. (P+B). K.

Pagurus bernhardus (Linnaeus, 1758)

Pagurus bernhardus: Rathke, H. 1840. NL.

Pagurus bernhardus: Rathke, H. 1842. NL.

Eupagurus bernhardus: Sars, G.O. 1889. Z1-Z4.G

Pagurus bernhardus: Williamson, H.C. 1915. Z1.LS.PL.
$(\mathrm{P}+\mathrm{B})$.

Eupagurus bernhardus: Webb, G.E. 1921. Z1.Z4. (P).

Eupagurus bernhardus: Gurney, R. 1942. K.

* Pagurus bernhardus: MacDonald, J.D.; Pike, R.B.

Williamson, D.I. 1957. Z1-Z4.G. (L+R). K.

Pagurus bernhardus: Pike, R.B.; Williamson, D.I. 1958. Z1-

Z4.M. (B). K.

Pagurus bernhardus: Hong, S.Y. 1988. Z1-Z4.M. (L).

Pagurus carneus Pocock, 1889

Pagurid larva: Sankarankutty, C. 1968. Z1-Z3. (P).

Pagurus cuanensis Bell, 1845

* Pagurus cuanensis: MacDonald, J.D.; Pike, R.B.;

Williamson, D.I. 1957. Z1-Z4.G. (L+P+R). K.

Pagurus cuanensis: Pike, R.B.; Williamson, D.I. 1958. Z1-

Z4.M. (B). K.

Pagurus cuanensis: Pike, R.B.; Williamson, D.I. 1960. K.

Pagurus cuanensis: Barnich, R. 1996. Z1-Z4.PL. (P+B). K.

Pagurus excavatus (Herbst, 1791)

Pagurus excavatus: Williamson, H.C. 1915. Z1. (B)

Pagurus alatus: Pike, R.B.; Williamson, D.I. 1960. Z1-Z4. (P)

* Pagurus alatus: Bookhout, C.G. 1972. Z1-Z4.G. (L).

Pagurus excavatus: Barnich, R. 1996. Z1-Z4. (P+B). K.

Pagurus forbesii Bell, 1845

Pagurus forbesii: Williamson, H.C. 1915. NE. (P).

Pagurus sculptimanus: MacDonald, J.D.; Pike, R.B.

Williamson, D.I. 1957. Z1. (P). K.

Pagurus forbesii ?: Pike, R.B.; Williamson, D.I. 1958. Z1.

(B). K.

Pagurus forbesii: Barnich, R. 1996. Z1.Z2. (B). K.

Pagurus prideauxi Leach, 1815

Eupagurus prideauxii: Mayer, P. 1877. NL.

Pagurus prideauxi: Williamson, H.C. 1915. Z1-Z4. (P+B).

Pagurus prideauxi: MacDonald, J.D.; Pike, R.B.; Williamson,

D.I. 1957. Z1-Z4.M. (L+P+R). K.

Pagurus prideauxi: Pike, R.B.; Williamson, D.I. 1958. Z1-

Z4.M. (B). K.

Pagurus prideauxi: Pike, R.B.; Williamson, D.I. 1960. K.

* Pagurus prideauxi: Goldstein, B.; Bookhout 1972. Z1-Z4.G. (L).

Pagurus prideaux: Hong, S.Y. 1988. Z1-Z4.M. (L).

Pagurus prideaux: Barnich, R. 1996. Z1-Z4.PL. (P+B). K.

Spiropagurus elegans Miers, 1881

* Spiropagurus elegans: Dechancé, M. 1962a. Z1-Z4.M. (L+P).

Spiropagurus elegans: Barnich, R. 1996. PL. K.

Family Parapaguridae S.I. Smith, 1882

Parapaguridae: Boschi, E.E. 1981. CG.

Parapagurus $s p$

Glaucothoe sp.: Bouvier, E.L. 1891. M. (P).

Anebocaris: Legendre, R. 1940. M. (P).

Larve Diaphroropus: Legendre, R. 1940. Z5. (P).

Glaucothoe peronii: Gurney, R. 1942. G. (P).

? Parapagurus pilosimanus: Pike, R.B.; Williamson, D.I.

1958. Z1. (B).

Parapagurus sp. 5: Saint Laurent - Dechancé, M. 1964. Z4. (P).

Parapagurus: Saint Laurent - Dechancé, M. 1964. CG.

Parapagurus ASM 4: Rice, A.L.; Williamson, D.I. 1977. Z1. (P).

Parapagurus pilosimanus S.I. Smith, 1879

Parapagurus sp. 1: Saint Laurent-Dechancé, M. 1964. Z1-Z5. (P).

Family Galatheidae Samouelle, 1819

Anomuran: Claus, C. 1861. Z. (P).

Galatheidae: Webb, G.E. 1921. CG.

Galatheidae: Gurney, R. 1942. Z2. (P).

Galatheidae: Boschi, E.E. 1981. CG.

Galatheidae: Ingle, R.W. 1992. CG. (B).

Galatheidae: Barnich, R. 1996. PL. (K)

Galathea sp

Galathea: Bate, C.S. 1878. Z. (P).

Pagurus: Claus, C. 1876. Z4. (P). 
Galathea (squamifera): Claus, C. 1885. Z4. (P).

Galathea: Gurney, R. 1942. CG.

Galathea sp.: Al-Kholy, A.A. 1959. Z1-Z3.M. (P).

Galathea: Pike, R.B.; Williamson, D.I. 1972. M. (B). K.

Galathea: Thiriot, A. 1974. Z1-Z4. CG.

Galathea ASM 5: Rice, A.L.; Williamson, D.I. 1977. Z2. (P). Galathea ASM 6: Rice, A.L.; Williamson, D.I. 1977. Z3. (P) Galathea ASM 7: Rice, A.L.; Williamson, D.I. 1977. Z4. (P). Galathea ASM 8: Rice, A.L.; Williamson, D.I. 1977. Z4. (P). Galathea: Barnich, R. 1996. Z1-Z5.PL. (P+B).

Galathea dispersa Bate, 1859

Galathea nexa: Sars, G.O. 1889. Z1. (L).

Galathea dispersa: Williamson, H.C. 1915. Z1. (P+B).

* Galathea dispersa: Lebour, M.V. 1930. Z1-Z5. (L+P).

Galathea dispersa: Bull, H.O. 1937. (B). K

Galathea dispersa: Kurian, C.V. 1956. (P). K.

Galathea dispersa: Pike, R.B.; Williamson, D.I. 1972.

Z1.LS.M. (B). K.

Galathea dispersa: Barnich, R. 1996. Z1-Z5.PL. (P+B). K.

Galathea intermedia Lilljeborg, 1851

Galathea intermedia: Stuxberg, A. 1874. NL.

Galathea intermedia: Sars, G.O. 1889. LS. (P).

Galathea intermedia: Williamson, H.C. 1915. Z1.LS. (B).

Galathea intermedia: Lebour, M.V. 1931a. Z1-Z4. (P+L).

Galathea intermedia: Kurian, C.V. 1956. K.

Galathea intermedia: Pike, R.B.; Williamson, D.I. 1972.

Z1.LS.M. (B). K.

* Galathea intermedia: Christiansen, M.E.; Anger, K. 1990.

Z1-Z5.M. (L).

Galathea intermedia: Barnich, R. 1996. Z1-Z4. (P+B). K.

Galathea nexa Embleton, 1834

Galathea nexa: Bull, H.O. 1937. Z1.Z2. CG. (P). K.

Galathea nexa: Pike, R.B.; Williamson, D.I. 1972. Z1. (B). K.

Galathea nexa: Barnich, R. 1996. Z1. (P+B). K.

Galathea squamifera Leach, 1814

Galathea squamifera: Sars, G.O. 1889. Z1. (L).

Galathea squamifera: Williamson, H.C. 1915. Z1. (B).

Galathea squamifera: Webb, G.E. 1921. Z1. (P).

* Galathea squamifera: Lebour, M.V. 1931a. Z1.Z3.Z4. (L+P).

Galathea squamifera: Bull, H.O. 1937. (B). K.

Galathea squamifera: Gurney, R. 1942. Z1. (B).

Galathea squamifera: Kurian, C.V. 1956. (P). K.

Galathea squamifera: Pike, R.B.; Williamson, D.I. 1972.

Z1.LS.M. (B). K.

Galathea squamifera: Barnich, R. 1996. Z1. (P+B). K.

Galathea strigosa (Linnaeus, 1767)

Galathea strigosa: Williamson, H.C. 1915. Z1. (P).

* Galathea strigosa: Lebour, M.V. 1930. Z1-Z4. (L+P).

Galathea strigosa: Bull, H.O. 1937. K.

Galathea strigosa: Gurney, R. 1942. Z4. (B).

Galathea strigosa: Kurian, C.V. 1956. (P). K.

Galathea strigosa: Pike, R.B.; Williamson, D.I. 1972.

Z1.LS.M. (B). K.

Galathea strigosa: Barnich, R. 1996. Z1. (P+B). K.

Munida sp.

Munida: Cano, G. 1893. PS.LS. (P).

Munida: Huus, J. 1935. Z1-Z4 CG.

Munida: Gurney, R. 1942. CG.

Munida: Pike, R.B.; Williamson, D.I. 1972. M. (B). K.

Munida ASM 9: Rice, A.L.; Williamson, D.I. 1977. Z1. (P).

Munida: Boschi, E.E. 1981. CG.

Munida sp.: Barnich, R. 1996. K.

Munida intermedia A. Milne Edwards and Bouvier, 1899

Munida intermedia sarsi: Pike, R.B.; Williamson, D.I. 1972.

Z1.LS. (B). K.

Munida sarsi Huus, 1935

Munida rugosa: Sars, G.O. 1889. Z1.LS. (P)

Munida sarsi: Huus, J. 1935. Z1. (L).

Munida rugosa (Fabricius, 1775)
Galathea rugosa: Rathke, H. 1840. NL.

Galathea rugosa: Williamson, H.C. 1915. Z1.LS. (P)

Munida banffica: Lebour, M.V. 1930. Z1-Z4. (L+P).

(B). K

Munida rugosa: Pike, R.B.; Williamson, D.I. 1972. Z1-Z4.M

Munida rugosa: Thiriot, A. 1974. CG.

Munida tenuimana Sars, 1872

Munida tenuimana: Williamson, H.C. 1915. Z2. (P+B).

* Munida tenuimana: Huus, J. 1935. Z1-Z4. (L).

Munida tenuimana: Pike, R.B.; Williamson, D.I. 1972. Z1-Z4.

(B). K.

Munidopsis sp

Munidopsis: Pike, R.B.; Williamson, D.I. 1972. M. (B). K.

Munidopsis serricornis (Lovén, 1853)

Galathodes tridentata: Sars, G.O. 1889. Z1.LS. (L+P). Galathea tridentata: Williamson, H.C. 1915. Z1.LS. (B).

* Munidopsis tridentata: Samuelsen, T.J. 1972. Z1-Z3.M. (L). Munidopsis tridentata: Pike, R.B.; Williamson, D.I. 1972.

Z1.LS.M. (B). K.

Munidopsis tridentata: Thiriot, A. 1974. CG.

Family Porcellanidae Haworth, 1825

Porcellana sp.: Müller, F. 1862. CG.

Porcellanidae: Webb, G.E. 1921. CG.

Porcellanidae: Gurney, R. 1942. CG.

Porcellanidae: Boschi, E.E. 1981. CG.

Porcellanidae: Ingle, R.W. 1992. CG.

Pisidia sp.

Pisidia: Boschi, E.E. 1981. CG.

Pisidia sp.: Barnich, R. 1996. Z1.Z2. (P). K. (P).

Pisidia ASM 10: Rice, A.L.; Williamson, D.I. 1977. Z1.Z2.M.

Pisidia bluteli (Risso, 1816)

Porcellana bluteli: Bourdillon Casanova, L. 1956a. Z1.Z2.M. (P). Porcellana bluteli: Bourdillon Casanova, L. 1960. M. (P).

Porcellana bluteli: Seridji, R. 1989. CG.

Pisidia bluteli: Kaya, B.; Özel, I. 1992. Z1.Z2.M. (P+B).

Pisidia longicornis (Linnaeus, 1767)

Porcellana longicornis: Dujardin, F. 1843. Z1. (L).

Galathea: Gosse, P.H. 1856. Z2. (P).

Porcellana longicornis: Bate, C.S. 1878. Z1. (P).

Porcellana platycheles: Hesse, M. 1884. Z1. (P).

Porcellana longicornis: Sars, G.O. 1889. Z1.Z2. (L+P).

Porcellana longicornis: Williamson, H.C. 1915. Z1.M. (P+B).

Porcellana longicornis: Webb, G.E. 1921. Z1.Z2. (P).

Porcellana sp.: Gurney, R. 1942. Z2. (P).

$(\mathrm{L}+\mathrm{R})$

* Porcellana longicornis: Lebour, M.V. 1943. Z1.Z2.M.

Porcellana longicornis: Kurian, C.V. 1956. Z1.Z2. (P).

Porcellana longicornis: Le Roux, A. 1966. Z1.Z2.M. (L+P).

Pisidia longicornis: Pike, R.B.; Williamson, D.I. 1972. Z1.M.

(B). K.

Pisidia longicornis: Thiriot, A. 1974. CG.

Pisidia longicornis: Seridji, R. 1989. CG.

Pisidia longimana: Kaya, B.; Özel, I. 1992. Z1.Z2. (P+B).

Porcellana sp.

Porcellana larvae: Claus, C. 1876. Z. (P).

Porcellana sp.: Webb, G.E. 1921. LS. (P).

Porcellana: Boschi, E.E. 1981. CG.

Porcellana platycheles (Pennant, 1777)

Porcellana platycheles: Couch, R.Q. 1843. Z1. (L).

(P).

Porcellana (Polyonyx) macrocheles: Faxon, W.A. 1879a. Z2.

Porcellana platycheles: Williamson, H.C. 1915. Z1.M. (P+B). Porcellana platycheles: Webb, G.E. 1921. Z1. (P). Porcellana platycheles: Lebour, M.V. 1943. Z1.Z2.M. (L+R). Porcellana platycheles: Pike, R.B.; Williamson, D.I. 1972.

Z1.M. (B). K.

Porcellana platycheles: Thiriot, A. 1974. CG.

Porcellana platycheles: Seridji, R. 1989. CG. 
Porcellana platycheles: Ingle, R.W. 1992. Z1. (B).

Porcellana platycheles: Kaya, B.; Özel, I. 1992. Z1.Z2.M. $(\mathrm{P}+\mathrm{B})$.

Porcellana platycheles: Barnich, R. 1996. Z1.Z2. (P)

* Porcellana platycheles: González-Gordillo, J.I.; Cuesta, J.A.; Rodríguez, A. 1996. Z1.Z2.M. (L). K.

Family Albuneidae Stimpson, 1858

Albuneidae: Seridji, R. 1989. CG.

Albuneidae: Boschi, E.E. 1981. CG.

Albunea sp.

Albunea: Gurney, R. 1942. CG

Albunea Specie A: Gurney, R. 1942. NE. (P).

Albunea Specie B: Gurney, R. 1942. Z1. (P).

Albunea: Thiriot, A. 1974. CG.

Albunea: Boschi, E.E. 1981. CG.

Albunea carabus (Linnaeus, 1758)

Albunea carabus: Seridji, R. 1988. Z1-Z3. (P).

Infraorder BRACHYURA Latreille, 1802

Gonoplax rhomboides: Cano, G. 1891.M. (P).

Megalopa armata: Williamson, H.C. 1915. M. (P).

Brachyura: Lebour, M.V. 1924. CG.

Brachyura: Lebour, M.V. 1927. CG.

Brachyura: Aikawa, H. 1929. CG.

Brachyura: Gurney, R. 1942. CG.

Zoe G.M.2: Bourdillon Casanova, L. 1960. Z2. (P).

Brachyura: Rice, A.L. 1980. CG

Brachyura: Boschi, E.E. 1981. CG.

Brachyura: Roff, J.C.; Davidson, K.G.; Pohle, G.; Dadswell,

M.J. 1984. Z.M. K.

Brachyura: Ingle, R.W. 1992. CG.

Family Dromiidae De Haan, 1833

Dromiidae: Rice, A.L. 1980. CG.

Dromiacea: Seridji, R. 1989. CG.

Dromia personata (Linnaeus, 1758)

Dromia vulgaris: Mayer, P. 1877. NL.

Dromia: Boas, J.E.V. 1880. Z5.M. (R).

Dromia vulgaris: Gourret, P. 1884. Z1. (L).

Dromia vulgaris: Cano, G. 1893. Z1.Z5?.M. (L+R)

Dromia vulgaris: Williamson, H.C. 1915. Z1.Z5.M. (P).

Dromidia larraburei: Rathbun, M.J. 1923. M. (P).

Dromia vulgaris: Lebour, M.V. 1934a. Z1.Z2.Z5.M. (L+R).

Dromia vulgaris: Gurney, R. 1942. Z1-Z4. (B).

Dromia vulgaris: Kurian, C.V. 1956. Z1. (P).

Dromia personatus: Pike, R.B.; Williamson, D.I. 1958.

Z1.Z2.M. (B).

Dromia personatus: Pike, R.B.; Williamson, D.I. 1960a. Z1-

Z3. (P).

Dromia personatus: Rice, A.L.; Provenzano, A.J. 1966. Z1-

Z4.M. (B).

* Dromia personata: Rice, A.L.; Ingle, R.W.; Allen, E. 1970.

Z1-Z4.M. (L).

Dromia personata: Thiriot, A. 1974. CG.

Dromia personata: Ingle, R.W. 1992. Z1Z4.M. (B). K.

Dromia personata: Barnich, R. 1996. Z1-Z4. (P+B). K.

Family Homolidae de Haan, 1839

Homolidae: Rice, A.L. 1980. CG.

Homolidea: Seridji, R. 1989. CG.

Homola sp.

Homola sp.: Williamson, H.C. 1915. M. (P).

Homola: Williamson, D.I. 1965. CG.

Homola barbata (Fabricius, 1793)

Homola sp.: Cano, G. 1893. LS. (P).

Homola sp.: Thiele 1905. LS. (P).

Homola cuvieri: Williamson, H.C. 1915. LS. (P).

Homola barbata: Pike, R.B.; Williamson, D.I. 1960a. Z1.Z2. (P)

Homola barbata: Rice, A.L. 1964. M. (P)

Homola sp.: Rice, A.L.; Levetzow, K.G. 1967. Z2-Z6. (P)

* Homola barbata: Rice, A.L.; Provenzano, A.J. 1970. Z1-Z7. (L).

Homola barbata: Thiriot, A. 1974. CG.
Homola barbata: Rice, A.L. 1980. Z1.Z6. (B).

Homola barbata: Wear, R.G.; Fielder, D.R. 1985. Z1.M. (B).

Homola barbata: Barnich, R. 1996. K.

Paromola cuvieri (Risso, 1816)

Paromola cuvieri: Samuelsen, T.J. 1976. Z1. (L).

Paromola cuvieri: Wear, R.G.; Fielder, D.R. 1985. Z1. (B).

Paromola cuvieri: Ingle, R.W. 1992. Z1. (B).

Family Latreillidae Stimpson, 1858

Latreillia elegans Roux, 1830 Latreillia: Cano, G. 1893. LS.M. (P).

Latreillia ? elegans ASM 11: Rice, A.L.; Williamson, D.I.

1977. Z1.Z2. (P).

Latreillia elegans: Rice, A.L. 1982. M. (P).

Family Dorippidae MacLeay, 1838

Dorippidae: Rice, A.L. 1980. CG.

Dorippe ?armata ASM 12: Rice, A.L.; Williamson, D.I. 1977. Z1. (P).

Ethusa mascarone (Herbst, 1785)

Ethusa Mascerone: Cano, G. 1892. Z1.Z2.M. (L+P).

Ethusa mascerone: Williamson, H.C. 1915. Z1. (P).

Ethusa: Gurney, R. 1942. CG.

Ethusa mascarone: Kurian, C.V. 1956. Z1-Z3. (P).

Ethusa mascarone: Bourdillon Casanova, L. 1960. Z1-Z4. (P).

Ethusa mascarone: Heegaard, P. 1963. Z1. (L).

Ethusa mascarone: Paula, J. 1987. Z1.Z3.Z4. (P).

Ethusa mascarone: Barnich, R. 1996. K.

Medorippe lanata (Linnaeus, 1767)

Dorippe lanata: Mayer, P. 1877. NL.

Dorippe lanata: Cano, G. 1892. Z1.Z2. (L+P).

Dorippe lanata: Cano, G. 1894. Z4. (L+P).

Dorippe lanata: Boraschi, L. 1921. Z1. (P).

Dorippe lanata: Gilet, R. 1952. Z3.M. (P).

$(\mathrm{P}+\mathrm{B})$

rippe lanata: Bourdillon Casanova, L. 1960. Z1-Z4.M

Dorippe lanata: Heegaard, P. 1963. Z1. (L).

Medorippe lanata: Paula, J. 1987. Z1-Z4. (P).

Medorippe lanata: Paula, J. 1991. Z1-Z4. (L).

Family Calappidae De Haan, 1833

Calappidae: Rice, A.L. 1980. CG.

Calappa granulata: Seridji, R. 1989. Z1. (P).

Calappidae: Seridji, R. 1989. CG.

Calappa granulata ?: Seridji, R. 1993. Z1. (P).

Calappa granulata (Linnaeus, 1767)

Calappa granulata: Taishaku, H.; Konishi, K. 1995. Z1. (B). Calappa granulata: Guerao, G.; Abelló, P.; Cartes, J. 1998. M. (P). Calappa granulata: Guerao, G.; Abelló, P.; Torres, P. 1999.

Z1. (L).

Family Leucosiidae Samouelle, 1819

Leucosiidae: Lebour, M.V. 1928. CG.

Leucosiidae EM22: Williamson, D.I. 1967. Z2-Z4. (P).

Leucosiidae: Rice, A.L. 1980. CG.

Leucosiidae: Seridji, R. 1989. CG.

Ebalia sp.

Ebalia sp.: Jorgensen, O.M. 1925. Z1-Z4.M. (P).

Ebalia: Lebour, M.V. 1928. CG

Ebalia: Gurney, R. 1942. LS.M. (P).

(P).

Ebalia? nux ASM 13: Rice, A.L.; Williamson, D.I. 1977. Z1.Z4.

Ebalia sp. ASM 14: Rice, A.L.; Williamson, D.I. 1977. Z3. (P).

Ebalia cranchii Leach, 1817

Plagusia sp.: Cano, G. 1892. Z4. (P).

* Ebalia cranchii: Lebour, M.V. 1928. Z1-Z4.M. (P+R).

Ebalia cranchii: Kurian, C.V. 1956. Z3.Z4.M. (P).

Ebalia cranchii: Salman, S.D. 1982a. Z1.Z4. (L+B).

Ebalia nux A. Milne Edwards, 1883

Ebalia nux: Rice, A.L. 1980. Z1.Z4. (B). 
Ebalia nux: Rice, A.L. 1980a. Z1. (L).

Ebalia nux: Salman, S.D. 1982a. Z1.Z4. (B).

Ebalia nux: Ingle, R.W. 1992. Z1.Z4. (B). K.

Ebalia tuberosa (Pennant, 1777)

Ebalia sp.: Williamson, H.C. 1915. Z1. (P).

Ebalia tuberosa: Lebour, M.V. 1928. Z1-Z4.M. (L+P).

Ebalia tuberosa: Lebour, M.V. 1928a. Z1-Z4.M. (L).

Ebalia tuberosa: Kurian, C.V. 1956. Z1.Z4.M. (P).

* Ebalia tuberosa: Salman, S.D. 1982a. Z1-Z4.M. (L).

Ebalia tuberosa: Wear, R.G.; Fielder, D.R. 1985. M. (B).

Ebalia tuberosa: Ingle, R.W. 1992. Z1-Z4.M. (B). K.

Ebalia tumefacta (Montagu, 1808)

* Ebalia tumefacta: Salman, S.D. 1982a. Z1-Z4. (L).

Ebalia tumefacta: Paula, J. 1987. M. (P).

Ebalia tumefacta: Ingle, R.W. 1992. Z1-Z4. (B). K.

Ilia nucleus (Linnaeus, 1758)

Planes sp.: Cano, G. 1892. Z1.Z2.Y.M. (L+P).

Ilia nucleus: Boraschi, L. 1921. Z1. (P).

Ilia nucleus: Bourdillon Casanova, L. 1960. Z1-Z3. (P).

Ilia nucleus: Heegaard, P. 1963. Z1. (L).

Family Majidae Samouelle, 1819

Stenorhynchus phalangium: Lo Bianco, S. 1904. M. (P).

Majidae: Lebour, M.V. 1928. CG.

Hyas araneus: Frost, N. 1936. M. (P).

NRS 47: Williamson, D.I. 1970. Z2. (P).

NRS 48: Williamson, D.I. 1970. Z1. Z2. (P).

NRS 49: Williamson, D.I. 1970. Z2. (P).

Majinae ASM 15: Rice, A.L.; Williamson, D.I. 1977. Z2. (P).

Acanthonychinae: Ingle, R.W. 1979a. CG.

Inachinae: Ingle, R.W. 1979a. CG.

Oregoniinae: Ingle, R.W. 1979a. CG.

Pisinae: Ingle, R.W. 1979a. CG.

Majinae: Ingle, R.W. 1979a. CG.

Majidae: Rice, A.L. 1980. CG.

Majidae: Boschi, E.E. 1981. CG

Oxyrhyncha: Ingle, R.W. 1982. Z.M. K.

Majidae S10: Paula, J. 1987. Z1. (P).

Majidae S11: Paula, J. 1987. Z2. (P).

Pisinae S8: Paula, J. 1987. Z1. (P).

Pisinae S9: Paula, J. 1987. Z1. (P).

Acanthonyx lunulatus (Risso, 1816)

Acanthonyx lunulatus: Cano, G. 1893a. Z2. (P).

Acanthonyx lunulatus: Boraschi, L. 1921. Z1. (P).

Acanthonyx lunulatus: Bourdillon Casanova, L. 1960. Z1.Z2.

(P).

Acanthonyx lunulatus: Heegaard, P. 1963. Z1. (L)

Acanthonyx lunulatus: Yang, W.T. 1968. CG (B).

* Acanthonyx lunulatus: Guerao, G.; Abelló, P. 1996a.

Z1.Z2.M. (L).

Achaeus sp.

Larva A (Achaeus?): Boraschi, L. 1921. Z1. (P).

Achaeus: Lebour, M.V. 1928. CG.

Achaeus spp.: Bourdillon Casanova, L. 1960. M. (P).

Achaeus: Ingle, R.W. 1982. Z.M. CG.

Achaeus cranchii Leach, 1817

* Achaeus cranchii: Bocquet, C. 1954. Z1.Z2.M. (L).

Achaeus cranchii: Paula, J. 1987. Z1.Z2. (P).

Achaeus cranchii: Ingle, R.W. 1992. Z1.Z2.M. (B). K.

Dorhynchus thomsoni Thomson, 1873

"Remarkable Zoea": Williamson, D.I. 1960a. Z2. (P).

Fam. nov.?: Williamson, D.I. 1974. Z1?. (P).

? Dorhynchus thomsoni: Rice, A.L. 1980. Z1.Z2. (P).

? Dorhynchus: Williamson, D.I. 1982. Z2. (B). K.

* Dorhynchus thomsoni: Williamson, D.I. 1982. Z1.Z2.M.

(R). K.

Dorhynchus thomsoni: Ingle, R.W. 1992. Z1.Z2.M. (B).

Eurynome sp.

Eurynome sp.: Cano, G. 1893a. CG.

Eurynome: Lebour, M.V. 1928. CG.
Eurynome: Ingle, R.W. 1982. Z.M. CG.

Eurynome aspera (Pennant, 1777)

Eurynome sp.: Cano, G. 1893a. Z1. (P).

Eurynome aspera: Gurney, R. 1924. Z1. (L)

Eurynome aspera: Lebour, M.V. 1928. Z1.Z2.M. (L+R).

* Eurynome aspera: Salman, S.D. 1982. Z1.Z2.M. (L).

Eurynome aspera: Wear, R.G.; Fielder, D.R. 1985. Z2.M. (B).

Eurynome aspera: Hong, S.Y. 1988. Z1.Z2.M. (L).

Eurynome aspera: Ingle, R.W. 1992. Z1.Z2.M. (B).

Eurynome spinosa Hailstone, 1835

Eurynome spinosa: Ingle, R.W. 1992. Z1.Z2.M. (B).

Herbstia sp.

Herbstia sp.: Cano, G. 1893a. CG.

Herbstia condyliata (Fabricius, 1787)

Herbstia condyliata: Cano, G. 1893a. Z2.M. (P).

Herbstia condyliata: Bourdillon Casanova, L. 1960. Z1. (P).

Inachus sp.

Inachus sp.: Cano, G. 1893a.

Megalopa niga: Williamson, H.C. 1915. M. (P).

Inachus: Lebour, M.V. 1928. CG.

Inachus spp.: Bourdillon Casanova, L. 1960. M. (P).

Inachus: Ingle, R.W. 1982. Z.M. CG.

Inachus S12: Paula, J. 1987. Z1. (P).

Inachus dorsettensis (Pennant, 1777)

Inachus scorpio: Claus, C. 1876. Z1. (P).

Inachus dorsettensis: Lebour, M.V. 1927. Z1.Z2.M. (L).

Inachus dorsettensis: Lebour, M.V. 1928. Z1.Z2.M. (L).

Inachus dorsettensis: Lebour, M.V. 1933. Z1.Z2.M. (L).

Inachus dorsettensis: Heegaard, P. 1963. Z1. (L).

* Inachus dorsettensis: Ingle, R.W. 1977. Z1.Z2.M. (L).

Inachus dorsettensis: Rice, A.L. 1980. Z1.Z2. (B).

Inachus dorsettensis: Hong, S.Y. 1988. Z1.Z2.M. (L).

Inachus dorsettensis: Ingle, R.W. 1992. Z1.Z2.M. (B)

Inachus leptochirus Leach, 1817

* Inachus leptocheirus: Lebour, M.V. 1928. Z1.Z2. (L+R).

Inachus leptochirus: Ingle, R.W. 1992. Z1.Z2.M. (B).

Inachus phalangium (Fabricius, 1775)

Inachus dorynchus: Gourret, P. 1884. Z1. (L).

Inachus dorynchus: Williamson, H.C. 1915. Z1. (B).

* Inachus dorhynchus: Lebour, M.V. 1928. Z1.Z2.M. (L+R).

Inachus thoracicus (Roux, 1830)

Inachus scorpio: Cano, G. 1893a. CG.

Inachus thoracicus: Heegaard, P. 1963. Z1. (L).

Lissa sp.

Lissa sp.: Cano, G. 1893a. CG.

Lissa chiragra (Herbst, 1783)

Lissa sp.: Cano, G. 1893a. Z1.Z2.M. (P).

Lissa chiragra: Boraschi, L. 1921. Z1. (P).

Lissa chiragra: Bourdillon Casanova, L. 1960. Z1.Z2. (L).

Lissa chiragra: Heegaard, P. 1963. Z1. (L).

Macropodia sp.

Stenorhynchus phalangium: Cano, G. 1893a. Z1.M.

Macropodia: Lebour, M.V. 1928. CG.

Macropodia sp.: Bourdillon Casanova, L. 1960. M. (P).

Macropodia: Ingle, R.W. 1982. Z.M. CG.

Macropodia S13: Paula, J. 1987. Z1.Z2.M. (P).

Macropodia deflexa Forest, 1978

Macropodia aegyptia: Lebour, M.V. 1928. Z1.Z2. (L+R).

Macropodia linaresi Forest and Zariquiey, 1964

Macropodia linaresi: Guerao, G.; Abelló, P.; Torres, P. 1998. Z1. (L)

Macropodia longipes (A. Milne Edwards and Bouvier, 1899)

(L). 
Macropodia rostrata (Linnaeus, 1761)

Macropodia phalangium ?: Thompson, J.V. 1835. Z1. (L).

? Stenorhynchus phalangium: Cano, G. 1893a. Z1.M. (P).

Inachus rostratus: Williamson, H.C. 1915. Z1.M. (P).

Macropodia rostratus: Lebour, M.V. 1928. Z1.Z2.M. (L+R).

* Macropodia rostrata: Ingle, R.W. 1982. Z1.Z2.M. (L).

Macropodia rostrata: Ingle, R.W. 1992. Z1.Z2.M. (B).

Macropodia tenuirostris (Leach, 1814) Macropodia longirostris: Lebour, M.V. 1927. Z1.Z2.M. (L). Macropodia longirostris: Lebour, M.V. 1928. Z1.Z2.M.

$(\mathrm{L}+\mathrm{R})$.

* Macropodia tenuirostris: Salman, S.D. 1981. Z1.Z2.M. (L). Macropodia tenuirostris: Ingle, R.W. 1982. Z1.Z2.M. (L). Macropodia tenuirostris: Ingle, R.W. 1992. Z1.Z2.M. (B).

Maja sp.

Maia: Lebour, M.V. 1928. CG.

Maja: Ingle, R.W. 1982. Z.M. CG.

Maja S7: Paula, J. 1987. Z1. (P).

Maja crispata (Risso, 1827)

Maia verrucosa: Claus, C. 1876. Z1. (P).

Maja verrucosa: Mayer, P. 1877. NL.

Maja verrucosa: Cano, G. 1893a. Z1.Z2.M. (L).

Maia verrucosa: Williamson, H.C. 1915. Z1.LS.PL. (B)

Maia verrucosa: Heegaard, P. 1963. Z1. (L).

Maja verrucosa: Seridji, R. 1989. CG.

Maja crispata: Neumann, V. 1993. Z1. (L).

Maja goltziana d'Oliveira, 1888

* Maja goltziana: Paula, J. 1988. Z1.Z2.M. (L).

Maja squinado (Herbst, 1788)

Maia squinado: Schlegel 1911. Z1.Z2.M. (L).

Maia squinado: Williamson, H.C. Z1.Z2.PL. (B). 1915.

Maia squinado: Lebour, M.V. 1927. Z1.Z2.M. (L).

Maia squinado: Lebour, M.V. 1928. Z1.Z2.M. (L+R).

Maja squinado: Gurney, R. 1942. Z1.Z2.M. (B).

Maia squinado: Kurian, C.V. 1956. Z1. (P).

Maja squinado: Bourdillon Casanova, L. 1960. M. (P).

Maja squinado: Yang, W.T. 1968. M. (B).

Maja squinado: Paula, J. 1985. Z1. (L).

* Maja squinado: Clark, P.F. 1986. Z1.Z2.M. (L)

Maja squinado: Paula, J. 1988. Z1.Z2.M. (B).

Maja squinado: Seridji, R. 1989. CG.

Maja squinado: Ingle, R.W. 1992. Z1.Z2.M. (B). K.

Pisa sp.

Pisa sp.: Cano, G. 1893a. CG.

Pisa: Ingle, R.W. 1982. Z.M. CG.

Pisa armata (Latreille, 1803)

Inachus tetraodon: Williamson, H.C. 1915. Z1.M. (P).

Pisa biaculeata: Lebour, M.V. 1931b. Z1.Z2. (L).

Pisa gibbsi: Bourdillon Casanova, L. 1960. M. (P).

Pisa armata: Heegaard, P. 1963. Z1. (L).

Pisa armata: Ingle, R.W.; Clark, P.F. 1980. Z1.Z2.M. (L).

Pisa armata: Rice, A.L. 1980. Z1. (B).

Pisa armata: Hong, S.Y. 1988. Z1.Z2.M. (L).

Pisa armata: Ingle, R.W. 1992. Z1.Z2.M. (B). K.

* Pisa armata: Rodríguez, A. 1997. Z1.Z2.M. (L).

Pisa nodipes (Leach, 1815)

Pisa sp. B: Bourdillon Casanova, L. 1960. Z1. (P).

Pisa nodipes: Heegaard, P. 1963. Z1. (L).

Pisa tetraodon (Pennant, 1777)

Pinnotheres veterum: Gourret, P. 1884. Z1. (L).

Pisa sp. A: Bourdillon Casanova, L. 1960. Z1. (P).

Pisa tetraodon: Heegaard, P. 1963. Z1. (L).

* Pisa tetraodon: Rodríguez, A. 1997. Z1.Z2.M. (L).

Rochinia $s p$.

Rochinia: Ingle, R.W. 1982. Z.M. CG.

Rochinia carpenteri (Thomson, 1873)

* Rochinia carpenteri: Ingle, R.W. 1979a. Z1.Z2.M. (L).
Rochinia carpenteri: Wear, R.G.; Fielder, D.R. 1985. Z1.M. (B). Rochinia carpenteri: Ingle, R.W. 1992. Z1.Z2.M. (B). K.

Family Parthenopidae MacLeay, 1838

Parthenopidae ASM 18: Rice, A.L.; Williamson, D.I. 1977.

Z1.Z2. (P).

Parthenopidae ASM 19: Rice, A.L.; Williamson, D.I. 1977.

Z2.Z3. (P).

Parthenopidae: Rice, A.L. 1980. CG.

Heterocrypta maltzami Miers, 1881

Heterocrypta maltzami: Thiriot, A. 1973. Z1. (L).

Parthenope sp.

Lambrus: Cano, G. 1893a. Z1-Z3. (P).

Lambrus sp.: Boraschi, L. 1921. Z1. (P).

? Parthenope sp. ASM 16: Rice, A.L.; Williamson, D.I. 1977.

Z2-Z4. (P).

Parthenope? massena: Rice, A.L. 1980. Z3. (B).

Species S3: Paula, J. 1987. Z1. (P).

Parthenope S14: Paula, J. 1987. Z1. (P).

Species S15: Paula, J. 1987. Z1. (P).

Parthenope angulifrons Latreille, 1825

Lambrus angulifrons: Heegaard, P. 1963. Z1. (L).

Parthenope angulifrons: Yang, W.T. 1971. Z1. (B).

Parthenope macrochelos (Herbst, 1790)

Parthenope macrochelos: Mura, M.; Pessani, D. 1994. Z1. (L).

Parthenope macrochelos: Guerao, G.; Abelló, P. 1999. Z1. (L).

Parthenope massena (Roux, 1830)

Lambrus massena: Gourret, P. 1884. Z1. (L).

Lambrus (Rhinolambrus) massena: Bourdillon Casanova, L.

1960. Z1.Z2. (P).

Lambrus massena: Heegaard, P. 1963. Z1. (L).

Parthenope massena: Yang, W.T. 1971. Z1. (B)

* Parthenope massena: Thiriot, A. 1973. Z1-Z4.M. (P)

Parthenope? massena: Rice, A.L.; Williamson, D.I. 1977.

Z2-Z5. (P).

Parthenope? massena: Rice, A.L. 1980. Z3. (B).

Parthenope massena: Paula, J. 1987. (P).

Parthenope massena: Ingle, R.W. 1992. Z1-Z5.M. (B). K.

Family Atelecyclidae Ortmann, 1893

Atelecyclidae: Rice, A.L. 1980. CG

Atelecyclidae: Boschi, E.E. 1981. CG.

Species S2: Paula, J. 1987. Z1.Z2. (P).

Atelecyclus $s p$

Larva C (Atelecyclus ?): Boraschi, L. 1921. Z1. (P).

Atelecyclus: Lebour, M.V. 1928. CG

Species S1: Paula, J. 1987. Z2.Z3.Z5.M. (P).

Atelecyclus undecimdentatus ?: Ingle, R.W. 1992. Z2.Z3.M. (B).

Atelecyclus rotundatus (Olivi, 1792)

Atelecyclus septemdentatus: Lebour, M.V. 1928. Z1-Z5.M. (L+P).

Atelecyclus septemdentatus: Kurian, C.V. 1956. M. (P).

Atelecyclus rotundatus: Bourdillon Casanova, L. 1960. Z4. (P).

Atelecyclus rotundatus: Bigot, J.L. 1979. Z1-Z5. (P).

* Atelecyclus rotundatus: Hong, S.Y.; Ingle, R.W. 1987. Z1-

Z5.M. (L)

Atelecyclus rotundatus: Ingle, R.W. 1992. Z1-Z5.M. (B). K.

Family Cancridae Latreille, 1803

Cancridae: Lebour, M.V. 1928. CG.

Cancridae: Rice, A.L. 1980. CG.

Cancer sp.

Cancer: Lebour, M.V. 1928. CG.

Cancer bellianus Johnson, 1861

Cancer sp. ASM 24: Rice, A.L.; Williamson, D.I. 1977. LS. (P).

Cancer bellianus ?: Ingle, R.W. 1992. Z5. (B)

Cancer pagurus Linnaeus, 1758

Cancer germanus: Linnaeus, C. 1767. M. (P).

Megalopa armata ?: Leach, W.E. 1817. M. (P). 
Cancer pagurus: Thompson, J.V. 1828. Z1. (L).

Cancer pagurus: Couch, R.Q. 1853. Z1.M. (L).

Cancer pagurus: Pearson, J.C. 1908. Z1. (L).

Cancer pagurus: Williamson, H.C. 1915. Z1-Z3. (B).

Cancer pagurus: Lebour, M.V. 1928. Z1-Z5.M. (L+P).

Cancer pagurus: Rice, A.L. 1975. Z1. (L).

Cancer pagurus: Bigot, J.L. 1979. Z1-Z5. (P).

Cancer pagurus: Rice, A.L. 1980. Z1. (B).

* Cancer pagurus: Ingle, R.W. 1981. Z1-Z5.M. (L).

Cancer pagurus: Ingle, R.W. 1992. Z1-Z5.M. (B). K.

Family Corystidae Samouelle, 1819

Corystidae: Lebour, M.V. 1928. CG.

Corystidae: Rice, A.L. 1980. CG.

Corystes sp.

Corystes: Lebour, M.V. 1928. CG.

Corystes cassivelaunus (Pennant, 1777)

Corystes cassivelaunus: Gurney, R. 1902. Z1-Z4.M. (P).

$(\mathrm{P}+\mathrm{B})$. Corystes cassivelaunus: Williamson, H.C. 1915. Z1-Z4.M.

Corystes cassivelaunus: Lebour, M.V. 1928. Z1-Z5.M. (P+R).

Corystes cassivelaunus: Gurney, R. 1942. LS. (B).

* Corystes cassivelaunus: Ingle, R.W.; Rice, A.L. 1971. Z1-

Z5.M. (L)

Corystes cassivelaunus: Rice, A.L. 1980. Z1.Z2.Z4.Z5. (B).

Corystes cassivelaunus: Ingle, R.W. 1992. Z1-Z5.M. (B). K.

Family Pirimelidae Alcock, 1819

Species S4: Paula, J. 1987. Z2. (P).

Pirimela denticulata (Montagu, 1808)

Pirimela denticulata: Kinahan, J.R. 1860. Z1. (L).

Pirimela denticulata: Cano, G. 1892a. M. (P).

Perimela sp.: Williamson, H.C. 1915. M. (P).

Perimela (Pirimela) denticulata: Williamson, H.C. 1915.

Z1.M. (P).

Larva B (Pirimela denticulata ?): Boraschi, L. 1921. Z1.Z2. (P).

Pirimela denticulata: Lebour, M.V. 1944. Z1.Z2. (L).

Pirimela denticulata: Bourdillon Casanova, L. 1956. Z1-

Z4.M. (L).

Pirimela denticulata: Bourdillon Casanova, L. 1960. Z1-

Z4.M. (P).

Pirimela denticulata: Paula, J. 1987. Z1-Z4.M. (P)

Pirimela denticulata: Ingle, R.W. 1992. Z1-Z4.M. (B). K.

Sirpus zariquieyi Gordon, 1953 $(\mathrm{L}+\mathrm{P})$.

Sirpus zariquieyi: Bourdillon Casanova, L. 1960. Z1-Z4.M.

Family Thiidae Dana, 1852

Thiidae: Lebour, M.V. 1928. CG

Thia scutellata (Fabricius, 1793)

Thia polita: Claus, C. 1876. Z1. (P).

Thia polita: Cano, G. 1892. Z1.Z3.Z4.M. (L+P).

Thia residua: Williamson, H.C. 1915. Z1.Z3.Z4.M. (B).

Thia polita: Lebour, M.V. 1928. Z2-Z4.M. (P).

* Thia scutellata: Ingle, R.W. 1984. Z1-Z4.M. (L).

Thia scutellata: Paula, J. 1987. Z1-Z4. (P).

Thia scutellata: Ingle, R.W. 1992. Z1-Z4.M. (B).

Family Geryonidae Colosi, 1923

Geryonidae: Rice, A.L. 1980. CG.

Geryon? longipes: Seridji, R. 1989. Z1-Z4. (P).

Geryon longipes A. Milne Edwards, 1882

* Geryon longipes: Guerao, G.; Abelló, P.; Castejón,

M.R.1996. Z1-Z4.M. (L).

Geryon trispinosus (Herbst, 1803) (L).

Geryon tridens: Brattergard, T.; Sankarankutty, C. 1967. Z1.

Bathynectes sp. A ASM 20: Rice, A.L.; Williamson, D.I.

1977. Z1. (P).

* Geryon tridens: Ingle, R.W. 1979. Z1-Z4.M. (L)

Geryon trispinosus: Terada, M. 1987. Z1-Z3. (L).
Geryon trispinosus: Ingle, R.W. 1992. Z1-Z4.M. (B).

Geryon trispinosus: Guerao, G.; Abelló, P.; Castejón,

M.R.1996. Z1-Z4.M. (B).

Family Portunidae Rafinesque, 1815

Portunus sp.: Williamson, H.C. 1915. (P).

Portunidae: Lebour, M.V. 1928. CG.

Portunus: Gurney, R. 1942. CG.

NRS 43: Williamson, D.I. 1970. Z3-Z5.M. (P).

NRS 44: Williamson, D.I. 1970. Z2 (P).

NRS 45: Williamson, D.I. 1970. Z3. (P)

Portunidae: Rice, A.L.; Ingle, R.W. 1975a. CG.

Portunidae: Rice, A.L. 1980. CG.

Portunidae: Boschi, E.E. 1981. CG.

Portunidae: Seridji, R. 1989. CG. K.

Bathynectes sp.

Bathynectes sp. A ASM 20: Rice, A.L.; Williamson, D.I.

1977. Z1. (P).

Bathynectes sp. B ASM 21: Rice, A.L.; Williamson, D.I.

1977. Z1. (P).

Bathynectes longipes (Risso, 1816)

Bathynectes longipes: Lebour, M.V. 1928. Z1. (L).

Bathynectes longipes: Lebour, M.V. 1931b. Z1. (L).

Bathynectes superba: Roberts, M.H. 1969. Z1-Z5. (L).

Bathynectes sp. B ASM 21: Rice, A.L.; Williamson, D.I.

1977. Z2. (P).

* Bathynectes longipes: Ingle, R.W. 1985a. Z1-Z5.M. (L).

Bathynectes longipes: Ingle, R.W. 1992. Z1-Z5.M. (B).

Carcinus aestuarii Czerniavsky, 1884

* Carcinus mediterraneus: Rice, A.L.; Ingle, R.W. 1975. Z1-

Z4.M. (L).

Carcinus maenas (Linnaeus, 1758)

Monoculus taurus: Slabber, M. 1778. Z2.M?. (P).

Monoculus taurus: Slabber, M. 1781. Z2.M?. (P).

Monoculus taurus: Latreille, P.A. 1802. Z2. (P).

Carcinus maenas: Du Cane, C. 1839a. Z1.

Carcinus maenas: Dalyell, J.G. 1851. Z1. (L).

Carcinus: Bate, C.S. 1859. Z1.Z3?.M. (L).

Carcinus maenas: Claus, C. 1861. Z. (P).

Carcinus maenas: Stuxberg, A. 1874. NL.

Carcinus maenas: Faxon, W.A. 1880. Z1. (L).

Carcinus maenas: Brooks, W.K. 1884. M. (R).

Portunus maenas: Williamson, H.C. 1915. Z1.Z4.M. (P).

Carcinus maenas: Jorgensen, O.M. 1918. Z1.Z2.Z3?.Z4.M. (L).

Carcinus moenas: Boraschi, L. 1921. Z1. (P).

Carcinus maenas: Lebour, M.V. 1928. Z1-Z4.M. (L+P).

Carcinus maenas: Shen, C.J. 1935. M. (P).

Carcinus maenas: Williams, B.G. 1968. Z1-Z4. (B)

* Carcinus maenas: Rice, A.L.; Ingle, R.W. 1975. Z1-Z4.M. (L).

Carcinus maenas: Rice, A.L. 1980. Z1.Z3.Z4. (B).

Carcinus maenas: Hong, S.Y. 1988. Z1-Z4.M. (L).

Carcinus maenas: Paula, J. 1988a. Z1-Z4.M. (B).

Carcinus maenas: Ingle, R.W. 1992. Z1-Z4.M. (B).

Liocarcinus sp.

Macropipus sp. A ASM 22: Rice, A.L.; Williamson, D.I.

1977. Z1.Z3. (P)

Macropipus sp. B ASM 23: Rice, A.L.; Williamson, D.I.

1977. Z3. (P).

Liocarcinus arcuatus (Leach, 1814)

Portunus arcuatus: Lebour, M.V. 1928. Z1.M. (L+R).

* Liocarcinus arcuatus: Clark, P.F. 1984. Z1-Z5. (L).

Liocarcinus arcuatus: Ingle, R.W. 1985a. Z1-Z5.M. (B).

Liocarcinus arcuatus: Ingle, R.W. 1992. Z1-Z5.M. (B).

Liocarcinus corrugatus (Pennant, 1777)

Portunus corrugatus: Lebour, M.V. 1928. Z1. (L).

Liocarcinus corrugatus: Clark, P.F. 1984. Z1-Z5. (L).

Macropipus corrugatus: Wear, R.G.; Fielder, D.R. 1985.

Z1.Z2.Z4. (L+P+B).

Liocarcinus corrugatus: Ingle, R.W. 1985a. Z1-Z5. (B).

Liocarcinus corrugatus: Hong, S.Y. 1988. Z1-Z5.M. (L).

Liocarcinus corrugatus: Ingle, R.W. 1992. Z1-Z5.M. (B). 
* Liocarcinus corrugatus: Kim, K.B.; Hong, S.Y. 1999. Z1Z5.M. (L).

Liocarcinus depurator (Linnaeus, 1758)

Portunus depurator: Stuxberg, A. 1874. NL.

Portunus depurator: Williamson, H.C. 1910. Z1. (L+P).

Portunus depurator: Björck, W. 1913a. Z3-Z5.M. (P).

Portunus depurator: Williamson, H.C. 1915. Z1-Z5.M. (P).

Megalopa montagui: Williamson, H.C. 1915. M. (P).

Portunus depurator: Lebour, M.V. 1928. Z1-Z3.M. (L+P).

Liocarcinus depurator: Clark, P.F. 1984. Z2-Z5. (L).

Liocarcinus depurator: Ingle, R.W. 1985a. Z1-Z5.M. (B).

Liocarcinus depurator: Ingle, R.W. 1992. Z1-Z5.M. (B).

Liocarcinus holsatus (Fabricius, 1798)

Portunus holsatus: Williamson, H.C. 1910. Z1-Z5.M. (L+P)

Portunus holsatus: Williamson, H.C. 1915. Z1-Z5.M. (P).

Portunus holsatus: Lebour, M.V. 1928. Z1.M. (L+P).

* Macropipus holsatus: Rice, A.L.; Ingle, R.W. 1975a. Z1-

Z5.M. (L).

Macropipus holsatus: Rice, A.L.; Ingle, R.W. 1978. Z1-Z5.M. (B)

Macropipus holsatus: Rice, A.L. 1980. Z5. (B).

Liocarcinus holsatus: Clark, P.F. 1984. Z1-Z5. (B).

Macropipus holsatus: Wear, R.G.; Fielder, D.R. 1985. M. (B).

Liocarcinus holsatus: Ingle, R.W. 1985a. Z1-Z5.M. (B).

Liocarcinus holsatus: Hong, S.Y. 1988. Z5.M. (L).

Liocarcinus holsatus: Ingle, R.W. 1992. Z1-Z5.M. (B).

Liocarcinus marmoreus (Leach, 1814)

Portunus marmoreus: Williamson, H.C. 1915. Z1.M. (P)

Portunus marmoreus: Lebour, M.V. 1928. Z1.M. (L+P).

* Macropipus marmoreus: Goldstein, B. 1971. Z1-Z5.M. (L).

Macropipus marmoreus: Rice, A.L.; Ingle, R.W. 1975a. Z1-

Z5.M. (B).

Macropipus marmoreus: Rice, A.L.; Ingle, R.W. 1978. Z1-

Z5.M. (B).

Liocarcinus marmoreus: Clark, P.F. 1984. Z1-Z5. (B)

Liocarcinus marmoreus: Ingle, R.W. 1985a. Z1-Z5.M. (B).

Liocarcinus marmoreus: Ingle, R.W. 1992. Z1-Z5.M. (B).

Liocarcinus pusillus (Leach, 1815)

Portunus pusillus: Lebour, M.V. 1928. Z1. (L).

* Macropipus pusillus: Rice, A.L.; Ingle, R.W. 1978. Z1-

Z5.M. (L).

Macropipus pusillus: Rice, A.L. 1980. Z2.Z5. (B).

Liocarcinus pusillus: Clark, P.F. 1984. Z1-Z5. (B)

Liocarcinus pusillus: Ingle, R.W. 1985a. Z1-Z5.M. (B)

Liocarcinus pusillus: Ingle, R.W. 1992. Z1-Z5.M. (B).

Liocarcinus vernalis (Leach, 1815)

Liocarcinus vernalis: Mura, M.; Pessani, D. 1994. Z1. (L)

Macropipus tuberculatus (Roux, 1830) (L).

Macropipus tuberculatus: Guerao, G.; Abelló, P. 1999. Z1-Z3.

Necora puber (Linnaeus, 1767)

Cancer rhomboidalis: Montagu, G. 1804. M. (P).

Portunus puber: Williamson, H.C. 1910. Z1-Z5.M. (L+P).

Portunus puber: Williamson, H.C. 1915. Z1-Z5.M. (L+P).

Portunus puber: Lebour, M.V. 1928. Z1-Z5.M. (L)

Macropipus puber: Bourdillon Casanova, E.L. 1960. CG.

* Macropipus puber: Rice, A.L.; Ingle, R.W. 1975a. Z1-

Z5.M. (L).

(B)

Macropipus puber: Rice, A.L.; Ingle, R.W. 1978. Z1-Z5.M.

Macropipus puber: Bigot, J.L. 1979. Z1-Z5. (P).

Liocarcinus puber: Clark, P.F. 1984. Z1-Z5. (B).

Liocarcinus puber: Ingle, R.W. 1985a. Z1-Z5.M. (B).

Liocarcinus puber: Mene 1987. Z1-Z5.M. (L).

Necora puber: Ingle, R.W. 1992. Z1-Z5.M. (B)

Polybius henslowii Leach, 1820

Polybius henslowi: Legendre, R. 1940. Z.M.(P).

Polybius henslowi: Lebour, M.V. 1944. Z1. (L).

Polybius henslowii: Sivertsen, E.; Holthuis, L.B. 1956. M. (P)

Polybius henslowi: Paula, J. 1985. Z1. (L).

Polybius henslowii: Ingle, R.W. 1992. Z1.M. (B).
Portumnus latipes (Pennant, 1777)

Pirimela denticulata?: Lebour, M.V. 1928. Z4.M. (P)

Portumnus latipes: Lebour, M.V. 1944. Z1.Z2.Z4.M. (L+P).

Portumnus latipes: Paula, J. 1987. Z1-Z4.M. (P).

* Portumnus latipes: Paula, J. 1988a. Z1-Z4.M. (L).

Portumnus latipes: Ingle, R.W. 1992. Z1-Z4.M. (B). K.

Portunus sp.

Portunus: Boschi, E.E. 1981. CG.

Xaiva biguttata (Risso, 1816)

Portumnus biguttatus: Lebour, M.V. 1928. M. (P).

Portumnus biguttatus: Lebour, M.V. 1944. M. (P).

Xaiva biguttata: Ingle, R.W. 1992. M. (B).

Family Goneplacidae MacLeay, 1838

Goneplacidae: Rice, A.L. 1980. CG.

Goneplax sp.

Gonoplax: Lebour, M.V. 1928. CG.

Goneplax rhomboides (Linnaeus, 1758)

Gonoplax rhomboides: Williamson, H.C. 1915. PL. (B).

Goneplax sp.: Boraschi, L. 1921. Z1. (P).

Gonoplax angulata: Caroli, E. 1927. Z1-Z4.M

Gonoplax rhomboides: Lebour, M.V. 1928. Z1-Z4.M. (L+P).

Gonoplax rhomboides: Kurian, C.V. 1956. Z2-Z4.M. (P).

Gonoplax angulata: Bourdillon Casanova, L. 1960. Z1.M.

$(\mathrm{P}+\mathrm{B})$.

Goneplax rhomboides ASM 28: Rice, A.L.; Williamson, D.I.

1977. Z2-Z4. (P).

Goneplax rhomboides: Ingle, R.W. 1979. CG.

Goneplax rhomboides: Rice, A.L. 1980. Z3. (B)

* Goneplax rhomboides: Ingle, R.W.; Clark, P.F. 1983. Z1-

Z4.M. (L).

Goneplax rhomboides: Ingle, R.W. 1992. Z1-Z4.M. (B). K.

Family Xanthidae Dana, 1851

Xanthidae: Lebour, M.V. 1928. CG.

Xanthide G.M.1: Bourdillon Casanova, L. 1960. Z1.Z2. (P).

Menippinae or Trapezinae ASM 26: Rice, A.L.; Williamson,

D.I. 1977. Z3.Z4. (P).

Xanthidae: Rice, A.L. 1980. CG.

Xanthidae: Boschi, E.E. 1981. CG.

Xanthidae: Martin, J.W. 1984. CG. (B)

Eriphia sp.

Eriphia: Martin, J.W. 1984. (B). K.

Eriphia verrucosa (Forskål, 1775)

Eriphia spinifrons: Cano, G. 1892a. CG.

Eriphia spinifrons: Boraschi, L. 1921. Z1. (P).

$(\mathrm{L}+\mathrm{P})$

Eriphia spinifrons: Bourdillon Casanova, L. 1960. Z1-Z3.

(L).

* Eriphia verrucosa: Lumare, F.; Gozzo, S. 1972. Z1-Z4.M.

Monodaeus couchi (Couch, 1851)

* Monodaeus couchi: Ingle, R.W. 1983. Z1-Z4.M. (L).

Monodaeus couchi: Hong, S.Y. 1988. Z1-Z4.M. (L).

Monodaeus couchi: Ingle, R.W. 1992. Z1-Z4.M. (B). K.

Panopeus africanus A. Milne Edwards, 1867

Panopeus ? africanus ASM 27: Rice, A.L.; Williamson, D.I.

1977. Z2. (P)

* Panopeus africanus: Rodríguez, A.; Paula, J. 1993a. Z1-

Z4.M. (L).

Pilumnus sp.

Pilumnus sp.: Cano, G. 1892a. Z1-Z3.M2. (L).

Pilumnus sp.: Williamson, H.C. 1915. Z1. (P).

Pilumnus: Lebour, M.V. 1928. CG.

Pilumnus ? inermis ASM 25: Rice, A.L.; Williamson, D.I.

1977. Z3. (P).

Pilumnus hirtellus (Linnaeus, 1761)

Pilumnus hirtellus?: Mayer, P. 1877. Z1. (L).

Pilumnus hirtellus: Williamson, H.C. 1915. Z1. (P+B). 
Pilumnus hirtellus: Boraschi, L. 1921. Z1. (P).

Pilumnus hirtellus: Lebour, M.V. 1928. Z1-Z4.M. (L).

Pilumnus hirtellus: Bourdillon Casanova, L. 1960. Z1. (L).

Pilumnus hirtellus: Salman, S.D. 1982b. Z1-Z4.M. (L+R).

* Pilumnus hirtellus: Ingle, R.W. 1983. Z1-Z4.M. (L).

Pilumnus hirtellus: Hong, S.Y. 1988. Z1-Z4.M. (L).

Pilumnus hirtellus: Ingle, R.W. 1992. Z1-Z4.M. (B). K.

Pilumnus spinifer H. Milne Edwards, 1834

Pilumnus spinifer: Gourret, P. 1884. Z1. (L).

Rhithropanopeus harrisii (Gould, 1841)

Rhithropanopeus harrisii: Connolly, C.J. 1925. Z1-Z4.M. (P)

Rithropanopeus harrisii: Chamberlain, N.A. 1962. Z1-Z4.M. (L).

Rithropanopeus harrisii: Hood, M.R. 1962. Z1-Z4.M. (L).

Rithropanopeus harrisii: Martin, J.W. 1984. K.

Rhithropanoeus harrisii: Ingle, R.W. 1992. Z1-Z4.M. (B).

Xantho sp.

Xantho sp.: Williamson, H.C. 1915. Z1-Z4.M. (P).

Xantho: Boraschi, L. 1921. Z1. (P).

Xantho: Lebour, M.V. 1928. CG.

Xantho incisus Leach, 1814

Xantho incisus: Lebour, M.V. 1928. Z1-Z4.M. (L).

Xantho incisus: Kurian, C.V. 1956. M. (P).

Xantho incisus: Bourdillon Casanova, L. 1960. Z1. (P).

* Xantho incisus: Ingle, R.W. 1983. Z1-Z4.M. (L)

Xantho incisus: Ingle, R.W. 1992. Z1-Z4.M. (B).

Xantho pilipes A. Milne Edwards, 1867

Xantho hydrophilus: Williamson, H.C. 1915. Z1. (P).

Xantho poressa (Olivi, 1792)

Xantho rivulosus: Gourret, P. 1884. Z1. (L).

Xantho hydrophilus: Lebour, M.V. 1928. Z1. (L)

Xantho poressa: Bourdillon Casanova, L. 1960. Z1. (P).

* Xantho poressa: Rodríguez, A.; Martin, J.W. 1997. Z1-Z4.M. (L).

Family Grapsidae MacLeay, 1838

Zoe GM2: Bourdillon Casanova, L. 1960. Z2. (P).

Grapsidae EM20: Williamson, D.I. 1967. LS. (P).

Plagusinae ASM 29: Rice, A.L.; Williamson, D.I. 1977. Z1. (P) Grapsinae ASM 30: Rice, A.L.; Williamson, D.I. 1977. Z3. (P). Grapsinae ASM 31: Rice, A.L.; Williamson, D.I. 1977. LS. (P). Grapsidae: Rice, A.L. 1980. CG.

Species S5: Paula, J. 1987. Z5. (P).

Brachynotus atlanticus Forest, 1957

* Brachynotus atlanticus: Rodríguez, A.; González-Gordillo,

J.I.; Cuesta, J.A. 1992. Z1-Z5.M. (L).

Brachynotus sexdentatus (Risso, 1827)

Brachynotus sexdentatus: Cano, G. 1891. Z1-Z3.M1.M2. (L).

Brachynotus sexdentatus: Bourdillon Casanova, L. 1960.

Z1.Z3-Z5.M. (L+P).

Brachynotus sexdentatus: Paula, J. 1987. Z2. (P).

* Brachynotus sexdentatus: Cuesta, J.A.; Schubart, C.;

Rodríguez, A. In press. Z1-Z5.M. (L).

Grapsus adscensionis (Osbeck, 1765)

Grapsus adscensionis: Cuesta, J.A.; González-Gordillo, J.I.;

Rodríguez, A. 1997. Z1. (L).

Pachygrapsus sp.

Paradesmarestia prototypa: Czerniavsky, V. 1880. M. (P).

Pachygrapsus marmoratus (Fabricius, 1787)

Pachygrapsus marmoratus: Cano, G. 1892. Z1.Z3?.Z4?.Z5.M.

$(\mathrm{L}+\mathrm{P})$.

Pachygrapsus marmoratus: Williamson, H.C. 1915.

Z1.Z2.Z5?. (P).

Pachygrapsus marmoratus: Hyman, O.W. 1924.

Z1.Z3?.Z5?.M. (L+P+B). K.

Pachygrapsus marmoratus: Bourdillon Casanova, L. 1960.

Z1. (P).

Pachygrapsus marmoratus: Paula, J. 1985. Z1. (L).

Pachygrapsus marmoratus: Ingle, R.W. 1987. Z1. (L).
Pachygrapsus marmoratus: Ingle, R.W. 1992. Z1.M. (B). K. Pachygrapsus marmoratus: Cuesta, J.A.; Rodríguez, A. 1994.

Z2. (L).

Pachygrapsus marmoratus: Guerao, G.; Abelló, P.; Cuesta,

J.A. 1997. M. (P).

Pachygrapsus marmoratus: Cuesta, J.A.; González-Gordillo,

J.I.; Rodríguez, A. 1997. Z1. (B).

Pachygrapsus marmoratus: Cuesta, J.A.; Rodríguez, A. In

press. ZIII-ZVI. (L).

Pachygrapsus maurus (Lucas, 1846)

Pachygrapsus maurus: Cuesta, J.A.; Rodríguez, A. 1994.

Z1.Z2. (L).

Pachygrapsus transversus (Gibbes, 1850)

Pachygrapsus transversus: Lebour, M.V. 1944a. Z1. (L)

Pachygrapsus transversus: Rossignol, M. 1957. M. (P).

Pachygrapsus transversus: Ingle, R.W. 1987. Z1. (L).

Pachygrapsus transversus: Cuesta, J.A.; Rodríguez, A. 1994.

Z2. (L).

Pachygrapsus transversus: Brossi-García, A.L.; Rodrígues,

M.D. 1997. Z1-Z7. (L).

Percnon gibbesi (H. Milne Edwards, 1853)

Pluteocaris sp.: Claus, C. 1876. Z. (P).

Percnon gibbesii: Lebour, M.V. 1944a. Z1.M. (L+P)

* Percnon gibbesi: Paula, J.; Hartnoll, R.G. 1989. Z1-Z6.M. (L+P).

Plagusia depressa (Fabricius, 1775)

* Plagusia depressa: Wilson, K.A.; Gore, R.H. 1980. Z1-Z5. (L).

Planes minutus (Linnaeus, 1758)

Nautilograpsus: Cano, G. 1892. Z1?.M.

Planes minutus: Hyman, O.W. 1924. Z1. (L).

Planes minutus: Lebour, M.V. 1944a. Z1.M. (L+P)

Planes minutus: Cuesta, J.A.; González-Gordillo, J.I.;

Rodríguez, A. 1997. Z1. (L).

Family Pinnotheridae De Haan, 1833

Pinnotheridae: Lebour, M.V. 1928. CG.

Pinnotheridae: Rice, A.L. 1980. CG.

Pinnotheridae: Boschi, E.E. 1981. CG.

Asthenognathus atlanticus Monod, 1933

* Tritodynamia atlantica: Bocquet, C. 1965. Z1-Z5.M. (L). Asthenognathus atlanticus: Ingle, R.W. 1992. Z1-Z5.M. (B). K.

Neopinnotheres pinnotheres (Linnaeus, 1758)

Pinnotheres Marioni: Gourret, P. 1888. Z1. (L+P).

Pinnotheres veterum: Hyman, O.W. 1924a. Z1. (B). K.

Pinnotheres veterum: Lebour, M.V. 1928. Z1.Z2.M. (L).

Pinnotheres veterum: Lebour, M.V. 1928a. Z1.Z2.M. (P+R).

Pinnotheres veterum: Gurney, R. 1942. Z1. (B).

* Pinnotheres pinnotheres: Atkins, D. 1955. Z1.Z2.M. (L+P).

Pinnotheres pinnotheres: Paula, J. 1987. Z1.Z2. (P).

Pinnotheres pinnotheres: Hong, S.Y. 1988. Z1.Z2.M. (L).

Pinnotheres pinnotheres: Seridji, R, 1989. Z2.(P).

Pinnotheres pinnotheres: Ingle, R.W. 1992. Z1.Z2.M. (B). K.

Pinnotheres $s p$.

Gen. Pinnotheres: Lebour, M.V. 1928. CG.

Pinnotheres pisum (Linnaeus, 1767)

Pinnotheres pisum: Williamson, H.C. 1915. Z1. (P).

Pinnotheres pisum: Hyman, O.W. 1924a. Z1. (B). K.

Pinnotheres pisum: Lebour, M.V. 1928. Z1.Z2?. (L+P).

Pinnotheres pisum: Lebour, M.V. 1928a. Z1. (L).

* Pinnotheres pisum: Atkins, D. 1955. Z1-Z4.M. (L+P).

Pinnotheres pisum: Rice, A.L. 1975. Z1. (L).

Pinnotheres pisum: Rice, A.L. 1980. Z1. (B).

Pinnotheres pisum: Hong, S.Y. 1988. Z1.Z5.M. (L).

Pinnotheres pisum: Ingle, R.W. 1992. Z1-Z4.M. (B). K.

Family Ocypodidae Rafinesque, 1815

Uca tangeri (Eydoux, 1835)

Uca tangeri: Paula, J. 1985. Z1. (L).

* Uca tangeri: Rodríguez, A.; Jones, D.A. 1993. Z1-Z5.M. (L). 


\section{REFERENCES}

Aikawa, H. - 1929. On larval forms of some Brachyura. Rec. Oceanogr. W. Japan, 2(1): 17-55.

Al-Kholy, A.A. - 1959. Larval stages of the three anomuran Crustacea. Publ. Mar. Biol. Sta. Al-Ghardaqa, 10: 83-89.

Al-Kholy, A.A. and El-Hawary, M.M. - 1970. Some penaeids of the red sea. Bull. Inst. Oceanogr. Fish. 1: 341-377.

Andersen, F.S. - 1962. The norway lobster in Faeroe waters. Meddr. Danm. Fisk. Havundres., 3 (9): 265-326.

Atkins, D. - 1955. The post-embryonic development of British Pinnotheres (Crustacea). Proc. Zool. Soc. London, 124: 687-715.

Balss, H. - 1925. Macrura der Deustcehn Tiefsee-Expedition. I. Palinura, Astacura und Thalassinidea. Wiss. Ergebn. Valdivia Exp., 20: 189-216.

Barnich, R. - 1996. The larvae of the Crustacea Decapoda (excl. Brachyura) in the plankton of the French Mediterranean Coast (Identification keys and systematic review). Ph. D. thesis, Univ. Münster, $189 \mathrm{pp}$.

Barnich, R. - 1996a. The identification of the penultimate larval stage of the Processidae (Crustacea, Decapoda, Caridea) from the French coast of the Mediterranean sea. Vie et Milieu, 46 $39-44$.

Bate, C.S. - 1859. On the development of decapod crustacea. Phil. Trans. R. Soc. London, 148: 589-605.

Bate, C.S. -1878 . Report on the present stage of our knowledge of Crustacea. IV. On development. Rep. Brit. Ass. Adv. Sci., 48 $193-209$.

Bate, C.S. - 1888. Report on the Crustacea Macrura dredged by H.M.S. Challenger during the years 1873-1876. Rep. Voy. Challenger, 24: 1-942.

Bernard, F. - 1953. Decapoda Eryonidae (Eryoneicus et Willemoesia). Dana Report, 37: 1-93.

Bhuti, G.S., Shenoy, S. and Sankolli, K.N. - 1977. Laboratory reared alpheid larvae of the genera Automate, Athanas and Synalpheus (Crustacea, Decapoda, Alpheidae). 588-600.

Bigot, J.L. - 1979. Identification des zoés de tourteau (Cancer pagurus L.).et d'etrille (Macropipus puber L.). Cons. Int. Explor. Mer., 17: 1-15.

Birshteyn, A.A. - 1938. Zemetka o Decapoda iz planktona, sobrannogo vo vremya plavaniya ledokolov Sadko i Litke v Arktike. Byull. M. O-Va Isp. Prirody Otd. Biol., 47: 199-209.

Björck, W. - 1911. Bidrag till kannendom om Decapodernas larvutveckling. I: Passiphaea. Ark. Zool., 7(15): 1-17.

Björck, W. - 1913. Beiträge zur Kenntnis der Decapodenmetamorphose. II. Über das postlarvale stadium von Calocaris macandreae Bell. Ark. Zool., 8: 1-8.

Björck, W. - 1913a. Biologish-Faunistische Untersuchungen dem resund I. Pantopoda, Mysidacea und Decapoda. Acta Univ. Lund., 9(17): 1-40.

Boas, J.E.V. - 1880. Studies over decapodernes slaegtskabsforhold K. Dans. vidensk. Selsk. Skr., 6: 25-210.

Bocquet, C. - 1954. Développement larvaire d'Achaeus cranchii Leach (Décapode, Oxyrhynque). Bull. Soc. Zool. France, 79(1): 50-56.

Bocquet, C. - 1965. Stages larvaires et juvéniles de Tritodynamia atlantica (Th. Monod) (=Asthenognathus atlanticus Th Monod) et position Systématique de ce crabe. Cah. Biol. Mar., 6: 407-418.

Bookhout, C.G. - 1972. Larval development of the hermit crab, Pagurus alatus Fabricius, reared in the laboratory (Decapoda, Paguridae). Crustaceana, 22(3): 213-238.

Boraschi, L. - 1921. Osservazione sulle larve dei Crostacei Decapodi brachyuri e anomuri. Mem. R. Com. Talassogr. Ital., 87: 1-32.

Boschi, E.E. - 1981. Larvas de Crustacea Decapoda. In: D. Boltovsky (ed). Atlas del zooplancton del Atlántico Sudoccidental y métodos de trabajo con zooplancton marino, pp. 699758. INIDEP, Mar del Plata.

Bourdillon Casanova, L. - 1956. Le développement larvaire de Pirimela denticulata Montagu (Crustacea Decapoda). Bull. Inst. Oceanogr. Monaco, 1073: 1-8.

Bourdillon Casanova, L. - 1956a. Note sur la prèsence de Porcellana bluteli (Risso) dans le golfe de Marseille et sur le développment larvaire de cette espèce. Cons. Int. Explor. Mer. 13: $225-232$

Bourdillon Casanova, L. - 1960. Le meroplancton du Golfe de Marseille: les larves de crustacés décapodes. Rec. Trav. Stat. Mar. Endoume, 30(18): 1-286.
Bouvier, E.L. - 1891. Les glaucothoes sont-elles des larves de Pagures?. Ann. Sci. Nat., 12: 65-82.

Bouvier, E.L. - 1905. Nouvelles observations sur les Glaucothoes. Bull. Mus. Oceanogr. Monaco, 2(51): 1-15.

Bouvier, E.L. - 1913. Sur les genres Pseudibacus et Nisto, et le stade natant des Crustacés décapodes macroures de la famille des Scyllarides. C. R.. Hebd. Acad. Sci., 156(22): 1643-1648.

Bouvier, E.L. - 1913a. Observations nouvelles sur le développment larvaire de la langouste commune Palinurus vulgaris Latreille. C. R. Acad. Sci. Paris: 457-463.

Bouvier, E.L. - 1914. Observations nouvelles sur les trachélifer, larves luciferiformes de Jaxea nocturna. J. Mar. Biol. Assoc. U. K., 10(2): 194-206.

Bouvier, E.L. - 1914a. Recherches sur le développemnet postembryonaire de la langouste commune (Palinurus vulgaris). $J$. Mar. Biol. Assoc. U. K., 10: 179-193.

Bouvier, E.L. - 1922. Observations complémentaires sur les crustacés décapodes (Abstraction faite des Carides) provenant des campagnes de S.A.S. le Prince de Monaco. Resul. Camp. Sci., 62: $107 \mathrm{pp}$

Bowman, T.E. and Abele, L.G. - 1982. Classification of the recent Crustacea. In: D.E. Bliss (ed.): The biology of Crustacea, Vol. 1: Systematics, the fossil record, and biogeography, pp.1-27. Academic Press, New York

Brattergard, T. and Sankarankutty, C. - 1967. On prezoea and zoea of Geryon tridens Kröyer (Crustacea, Decapoda). Sarsia, 26: 7-12.

Brooks, W.K. - 1880. The young of the crustacean Lucifer, a nauplius. Amer. Nat., 14: 806-808.

Brooks, W.K. - 1882. Lucifer, a study in morphology. Phil. Trans. R. Soc., 173: $57-137$.

Brooks, W.K. - 1884. On the rate of development of the common shore-crab (Carcinus maenas). Ann. Mag. Nat. Hist., 14: 202-207.

Brooks, W.K. - 1889. Notes on a Lucifer-like decapod larva from the west coast of Scotland. Proc. R. Soc. Edinb. 15: 420-423.

Brossi-García, A.L. and Domíngues, M. - 1997. Zoeal morphology of Pachygrapsus transversus (Gibbes) (Decapoda, Grapsidae) reared in the laboratory. Rev. Bras. Zool., $14: 803-819$.

Bull, H.O. - 1933. The newly-hatched larvae of Calocaris macandreae Bell. Rep. Dove Mar. Lab., 1(3): 48-50.

Bull, H.O. - 1937. Notes on the Bristish species of the genus Galathea Fab. Rep. Dove Mar. Lab., 3(4): 38-52.

Bull, H.O. - 1939. The newly-hatched larva of Spirontocaris pusiola (Kröyer). Rep. Dove Mar. Lab., 6(3): 43-44.

Burkenroad, M.D. - 1983. Natural classification of Dendrobranchiata, with a key to recent genera. Crust. issues, 3: 279-290.

Calafiore, N., Costanzo, G. and Giacobbe, S. - 1991. Mediterranean species of the genus Pontonia Latreille, 1829. I. Developmental stages of Pontonia pinnophylax (Otto, 1821) (Decapoda, Natantia, Pontoniinae) reared in the laboratory. Crustaceana, 60(1): 52-75.

Cano, G. - 1891. Sviluppo postembrionale dei Gonoplacidi. Atti Acad. Sci. Torino, 26: 639-648.

Cano, G. - 1891a. Sviluppo postembrionale della Gebia, Axius, Callianassa e Calliaxis. Morfologia dei Talassinidi. Boll. Soc. Nat. Napoli, 1: 5-30.

Cano, G. - 1891b. Sviluppo post-embrionale dello Stenopus spinosus Risso. Studio morfologico. Boll. Soc. Nat. Napoli, Sr.1, 5(2): 134-137.

Cano, G. - 1892. Sviluppo postembrionale dei Dorippidei, Leucosiadi, Corystoidei e Grapsidi. Mem. Soc. Ital. Sci. Nat., 8(4): $1-14$.

Cano, G. - 1892a. Sviluppo postembrionale dei Cancridi. Bull. Ent. Ital., 24: 146-158.

Cano, G. - 1893. Svilippo dei Dromidei. Att. Acad. Sci. Fis. Mat. Napoli, 6(2): 1-23.

Cano, G. - 1893a. Sviluppo e morfologia degli oxyrhynchi. Mitt. Zool. Stat. Neapel, 10: 527-583.

Cano, G. - 1894. Dorippe, studio morfologico. Att. Acad. Sci. Fis. Mat. Napoli, 6(9): 1-9.

Carayon, J. - 1942. Sur le stade glaucothoe du pagure Clibanarius misanthropus et sur l'établissement de la dissymétrie chez les pagures. Comparaison avec Glaucothoe Grimaldii. C. R. Seanc. Acad. Sci., 214: 387-389

Carli, A. - 1978. Chiave analitica per l'identificazione degli stadi larvali planctonici e del primo stadio postlarvale di Palaemon elegans, P. serratus, P. xiphias (Crustacea, Decapoda). Mem. Biol.Mar. Ocean., 8(5): 115122 .

Carli, A. and Marchi, M. - 1979. Osservazioni sugli stadi larvali 
planctonici di Palaemon xiphias Risso, 1816 allevati in laboratorio (Crustacea, Decapoda). Boll. Mus. Ist. Biol. Univ. Genova, 47: 5-20

Carli, A. and Vignola, S. - 1977. Osservazioni sugli stadi larvali planctonici di P. elegans Rathke, 1837 allevate in laboratorio. Boll. Pesca Pisc. Idrobiol., 34: 1-22.

Caroli, E. - 1918. Miersia clavigera Chun, stadio misidiforme di Lysmata seticaudata. Pub. Staz. Zool. Napoli, 2: 177-189.

Caroli, E. - 1924. Sviluppo larvale e primo stadio postlarvale della Jaxea nocturna Nardo (=Calliaxis adriatica Heller). Pub. Staz. Zool. Napoli, 5: 153-198.

Caroli, E. - 1926. La zoea dei Pontoniinae. Boll. Soc. Nat. Napoli, 37: $3-5$.

Caroli, E. - 1927. Sviluppo larvale della Gonoplax angulata (Pennant). Boll. Soc. Nat. Napoli, 38: 161-166.

Caroli, E. - 1946. Di un puerulus di Palinurus vulgaris pescato nel Golfo di Napoli. Pub. Staz. Zool. Napoli, 20: 152-157.

Cecchini , C. - 1928. Sopra alcuni forme larvali di Sergestes non comprese in serie larvali note. Att. Soc. Ital. Sci. Nat., 67: 144-149.

Chadwick, H.C. - 1905. Experiments on lobters rearing. Trans. Liverpool Biol. Soc., 19: 304-308.

Christiansen, M.E. and Anger, K. - 1990. Complete larval development of Galathea intermedia Lilljeborg reared in laboratory culture (Anomura, Galatheidae). J. Crust. Biol., 10(1): 87-111.

Clark, P.F. - 1984. A comparative study of zoeal morphology in the genus Liocarcinus (Crustacea, Brachyura, Portunidae). Zool. J. Linn. Soc., 82: 273-290.

Clark, P.F. - 1986. The larval stages of Maja squinado (Herbst, 1788) (Crustacea, Brachyura, Majidae) reared in the laboratory. J. Nat. Hist., 20: 825-836.

Claus, C. - 1861. Zur Kenntnis der Malacostracenlarven. Würzb. Naturw. Zeitschr., 2: 23-46.

Claus, C. - 1876. Untersuchungen zur Erforschung der Genealogischen Grundlage des Crustaceen-Systems. Wien, 114 pp.

Claus, C. - 1884. Zur kenntnis der kreislaufsorgane der Schizopoden und Decapoden. Arb. Zool. Inst. Univ. Wien, 5: 271-318.

Claus, C. -1885 . Neue beiträge zur morphologie der Crustaceen. Arb. Zool. Inst. Univ. Wien, 6: 1-108.

Connolly, C.J. - 1925. The larval stages and megalops of Rhithropanopeus harrisi (Gould). Contr. Can. Biol. Fish., 15: 329-334.

Costanzo, G., Calafiore, N. and Crescenti, N. - 1996. Mediterranean species of the genus Pontonia Latreille, 1829. II. Developmental stages of Pontonia flavomaculata Heller, 1864 (Decapoda, Natantia, Pontoniinae) reared in the laboratory. Crustaceana, 69(6): 773-781.

Couch, R.Q. - 1843. On the metamorphosis of the decapod Crustacea. Rep. R. Cornw. Polyth. Soc., 11: 28-43.

Couch, R.Q. - 1853. Notes on the metamorphosis of the common crab (C. pagurus). Trans. Nat. Hist. Antiq. Soc. Penzance, 2: 200-203.

Coutière, H. - 1898. Note sur le developpement de Synalpheus laevimanus Heller. Bull. Soc. Entom. France, 1898: 220-222.

Coutière, H. - 1906. Note sur la synonymie et le développement de quelques Hoplophoridae. Bull. Mus. Oceanogr. Monaco, 70: 1-20.

Coutière, H. - 1907. Sur quelques formes larvaires enigmatiques d'Eucyphotes, provenant des collections de S.A.R. le Prince de Monaco. Bull. Inst. Oceanogr. Monaco, 104: 1-70.

Criales, M.M. and McGowan, M.F. - 1993. Coastal oceanic planktonic distribution of natantia shrimps in the Florida Keys, USA. Rev. Biol. Trop., Supl. 41(1): 23-26.

Cuesta, J.A., González-Gordillo, J.I. and Rodríguez, A. - 1997. First zoeal stages of Grapsus adscensionis (Osbeck) and Planes minutus (Linnaeus) (Brachyura, Grapsidae) described from laboratory hatched material, with notes on larval characters of the Grapsinae. J. Nat. Hist., 31: 887-900.

Cuesta, J.A. and Rodríguez, A. - 1994. Early zoeal stages of Pachygrapsus marmoratus (Fabricius), P. transversus (Gibbes) and $P$. maurus (Lucas) (Decapoda, Brachyura, Grapsidae) reared in the laboratory. Sci. Mar., 58(4): 323-327.

Cuesta, J.A., Schubart, C. and Rodríguez, A. - In press. Zoeal stages of the intertidal crab Pachygrapsus marmoratus (Fabricius, 1787) (Brachyura, Grapsidae) reared in the laboratory. Hydrobiologia.

Cunningham, J.T. - 1891. On the development of Palinurus vulgaris. J. Mar. Biol. Ass. U. K., 2: 141-151.

Czerniavsky, V. - 1880. Megalopidea S. larvae anomuriformes crustaceorum brachyurorum. Trav. Soc. Entom. Russe, 11: 51-96.
Czerniavsky, V. - 1884. Crustacea Decapoda Pontica Littoralia. Materalia ad Zoographiam Ponticam Comparatam, II. Trans. Soc. Univ. Kharkow, 13: 1-268.

Dall, W., Hill, B.J., Rothlisberg, P.C. and Staples, D.J. - 1990. The biology of the Penaeidae. Adv. Mar. Biol., 27: 1-489.

Dalyell, J.G. - 1851.The powers of the Creator displayed in the Creation, Vol. I. London.

De Simon, M. - 1979. Primeros estadios larvarios de Pontocaris lacazei (Gourret) (Decapoda, Macrura, Crangonidae) obtenidos en laboratorio. Inv. Pesq., 43(2): 565-580.

Dechancé, M. - 1958. Caractérisation de la glaucothoe et des premiers stades Pagure chez Clibanarius misanthropus (Risso) (Crust. Décapode Anomoure). C. R. Hebd. Sean. Acad. Sci. 246: 839-842.

Dechancé, M. - 1961. Sur la morphologie externe des larves de Pagurides. I. Catapaguroides timidus (Roux). Crustaceana, 2 53-67.

Dechancé, M. - 1962. Remarques sur les premiers stades larvaires de plusieurs espèces indopacifiques du genre Dardanus (Crustacés, Décapodes, Pagurides). Bull. Mus. Nat. Hist. Nat., 34(1): $82-94$

Dechancé, M. - 1962a. Description des stades larvaires et de la glaucothoé de Spiropagurus elegans Miers. Bull. Mus. Nat. Hist. Nat., 34(5): 371-386.

Dechancé, M. and Forest, J. - 1958. Les glaucothoés de Catapaguroides timidus (Roux) et de Clibanarius erythropus (Latreille). Remarques sur le stade postlarvaire des pagurides. Bull. Soc.Zool. Fr., 83(2/3): 274-293

Dechancé, M. and Forest, J. - 1962. Sur Anapagurus bicorniger Milne Edwards et Bouvier et A. petiti sp. nov. (Crustacea, Decapoda, Paguridae). Bull. Mus. Nat. Hist. Nat., 2 ser., 34(4): 293-307.

Dohrn, A. - 1908. Zur kenntnis der Metamorphose von Sergestes arcticus Kr. Zool. Anz., 33: 303-331.

Dolgopolskaya, M.A. - 1969. Larvae of Decapoda Macrura and Anomura. In: Morduhai-Boltovskoi (ed.): Key to the fauna of the Black and Azov seas, 2: 307-362. (In Russian)

Du Cane, C. - 1839. Letter from Captain Du Cane, R.N., to the Ver. Leonard Jenyns, on the subject of the metamorphosis of Crustacea. Ann. Mag. Nat. Hist., 2(9): 178-180.

Du Cane, C. - 1839a. On the metamorphosis of the Crustacea. Ann. Mag. Nat. Hist., 3: 438-440.

d'Udekem d'Acoz, C. - 1999. Inventaire et distribution des crustacés décapodes de l'Atlantique nord-oriental, de la Méditerranée et des eaux continentales adjacentes au nord de $25^{\circ} \mathrm{N}$. Patrimoines naturels (MNHN / SPN), 40: 1-383.

Dujardin, F. - 1843. Observations sur les métamorphoses de la Porcellana longicornis, et description de la Zoé, qui est la larve de ce crustacé. C. R. Hebd. Seanc. Acad. Sci., 16: 1204-1207.

Ehrebaum, E. - 1890. Zur naturgeschichte von Crangon vulgaris Fabr. In: Studien über Bau, Entwichklung, Lebenweise und Fanguerhältnisse des Nordsee-Granat im Auftrage der Sektion für Küsten und Hochseefischerei des Deutschen Fischerei-Vereins. Berlin, 124 pp.

Elofsson, R. - 1959. A new decapod larva refered to Calocarides coronatus (Trybom). Publ. Biol. Sta. Espegrend, 26: 1-10.

Elofsson, R. - 1961. The larvae of Pasiphaea multidentata (Esmark) and Pasiphaea tarda (Kröyer). Sarsia, 4: 43-53.

Faxon, W.A. - 1879. On the development of Palaemonetes vulgaris. Bull. Mus. Comp. Zool. Harvard Coll., 5: 303-330.

Faxon, W.A. - 1879a. On some young stages in the development of Hippa, Porcellana, and Pinnixia. Bull. Mus. Comp. Zool. Harvard Coll., 5: 253-268.

Faxon, W.A. - 1880. On some points in the structure of the embryonic zoea. Bull. Mus. Comp. Zool. Harvard Coll., 6: 159-166.

Faxon, W. A. - 1882. Selections from embryological monographs. I. Crustacea. Mem. Mus. Comp. Zool. Harv., 9: 1-32.

Fedele, M. - 1926. La metamorfosi dal Phyllosoma dello Scyllarus arctus. Biol. Soc. nat. Napoli, 37: 215-233.

Fincham, A.A. - 1977. Larval development of british prawns and shrimps (Crustacea, Decapoda, Natantia). - 1. Laboratory methods and review of Palemon (Paleander) elegans Rathke, 1837. Bull. Br. Mus. Nat. Hist., 32(1): 1-28.

Fincham, A.A. - 1979. Larval development of british prawns and shrimps (Crustacea, Decapoda, Natantia). 3. Palaemon (Palaemon) longirostris Milne Edwards, 1837 and the effect of antibiotic on morphogenesis. Bull. Br. Mus. Nat. Hist., 37(1): 17-46.

Fincham, A.A. - 1983. Larval development of british prawns and 
shrimps (Crustacea, Decapoda, Natantia). 4. Palaemon (Palaemon) serratus (Pennant, 1777) and the functional morphology of swimming. Bull. Br. Mus. Nat. Hist., 44(2): 125-161.

Fincham, A.A. - 1985. Larval development of british prawns and shrimps (Crustacea, Decapoda, Natantia). 5. Palaemon (Palaemon) adspersus Rathke, 1837. Bull. Br. Mus. Nat. Hist., 48(4): 215-231.

Fincham, A.A. and Figueras, A.J. - 1986. Larval keys and diagnoses for the subfamily Palaemoninae (Crustacea, Decapoda, Palaemonidae) in the northeast Atlantic and aspects of functional morphology. J. Nat. Hist., 20: 203-224.

Fincham, A.A. and Williamson, D.I. - 1978. Crustacea Decapoda: larvae. VI. Caridea, Families: Palaemonidae and Processidae. Fich. Ident. Zoopl., 159/160: 1-8.

Frontier, S. - 1963. Notes sur quelques larves atypiques de Décapodes brachyoures récoltes dans le plankton de Nosy Bé. Cah. ORSTOM , 3: 31-59.

Frost, N. - 1936. Decapod larvae from Newfoundland waters. Fish. Res. Bull. Newfoundland, 3(2): 11-24.

García Raso, J.E. - 1996. Crustacea Decapoda (Excl. Sergestidae) from Ibero-Moroccan waters. Results of Balgim-84 Expediton. Bull. Mar. Sci., 58(3): 730-752.

Gilet, R. - 1952. Métazoé de Dorippe lanata (Linné) et sa mégalope. Vie et Milieu, 3(4): 415-420.

Goldstein, B. - 1971. Développement larvaire de Macropipus marmoreus (Leach) en laboratoire (Crustacea, Decapoda, Portunidae). Bull. Mus. Nat. Hist. Nat., 42(5): 919-943.

Goldstein, B. and Bookhout, C.G. - 1972. The larval development of Pagurus prideauxi Leach, 1814, under laboratory conditions (Decapoda, Paguridea). Crustaceana, 23(3): 263-281.

González-Gordillo, J.I., Cuesta, J.A. and Pablos, F. - 1990. Adiciones al conocimiento de los crustáceos decápodos de las zonas mediolitoral e infralitoral de las costas suratlánticas andaluzas (Suroeste España). I Brachyura. Cah. Biol. Mar, 31: 417-429.

González-Gordillo, J.I., Cuesta, J.A. and Rodríguez, A. - 1996. Studies on the larval development of northeastern Atlantic and Mediterranean Porcellanidae (Decapoda, Anomura). I Redescription of the larval stages of Porcellana platycheles (Pennant, 1777) reared under laboratory conditions. Helg. Meeresunt., 50: 517-531.

González-Gordillo, J.I. and Rodríguez, A. - 2000. Larval development of Philocheras fasciatus (Risso, 1816) (Decapoda, Caridea) reared in the laboratory, comparison with plankton larvae and occurence of accelerated development. J. Plank. Res., 22: 1909-1923.

González-Gordillo, J.I. and Rodríguez, A. - 2000. First larval stage of Scyllarus posteli Forest, 1963 and Processa macrodactyla Holthuis, 1952 (Crustacea, Decapoda) hatched in the laboratory. Ophelia, 53: 91-99.

González-Gordillo, J.I., dos Santos, A. and Rodríguez, A. - 2000. The complete larval development of Philocheras monacanthus (Holthuis, 1961) from laboratory culture, with an identification key to the European species of the genus (Decapoda, Caridea, Crangonidae). J. Crust. Biol., 20: 75-88.

Gordon, I. - 1964. On the larval genus Problemacaris Stebbing an its probable identity (Crustacea, Decapoda). Zool. Meded. Leiden, 39: 331-347.

Gosse, P.H. - 1856. Tenby, a sea-side holiday. London.

Gourret, P. - 1884. Considérations sur la fauna pélagique du Golfe de Marseille suivies d'une étude anatomique et zoologique de La Spadella Marioni espèce nouvelle de l'ordre des choetognathes (Leuckart). Ann. Mus. Hist. Nat. Marseille, 2(2): 1-175.

Gourret, P. - 1888. Études zoologiques sur quelques Crustacés parasites des Ascidies. Bibl. Ec. Haut. Etud. Sci. nat., 36(3): 1-64.

Gray, J.W. - 1969. Investigations of the basic life history of the red crab (Geryon quinquedens). Rhode Island Div. Conser. Proj. 3-46-R: 1-36.

Greenwood, J.G. and Fielder, D.R. - 1979a. A comparative study of the first and last zoeal stages of four species of Thalamita (Crustacea, Portunidae). Micronesica, 15: 304-309.

Guerao, G. - 1993. The larval development of a fresh-water prawn, Palaemonetes zariquieyi Sollaud, 1939 (Decapoda, Palaemonidae), reared in the laboratory. Crustaceana, 64(2): 226-241.

Guerao, G. and Abelló, P. - 1996. Description of the first larval stage of Polycheles typhlops (Decapoda, Eryonidea, Polychelidae). J. Nat. Hist., 30: 1179-1184.

Guerao, G. and Abelló, P. - 1996a. Larval development of Acanthonyx lunulatus (Brachyura, Majidae, Epialtinae). J. Plank.
Res., 18(7): 1185-1200.

Guerao, G. and Abelló, P. - 1997. Larval development of the spider crab Macropodia longipes (Brachyura, Majidae, Inachinae). J. Crust. Biol., 17(3): 459-471.

Guerao, G. and Abelló, P. - 1999. Morphology of the early zoeal stages of Macropipus tuberculatus (Roux, 1830) (Crustacea, Brachyura, Portunidae). J. Plank. Res., 21: 1993-2008.

Guerao, G., Abelló, P. and Cartes, J. - 1998. Morphology of the megalopa and first crab instar of the Shamefaced Crab Calappa granulata (Crustacea, Brachyura, Calappidae). Misc. Zool., 21: 37-47.

Guerao, G., Abelló, P. and Castejón, M.R. - 1996. Morphology of the larval stages of the deep-sea crab Geryon longipes (Brachyura, Geryonidae). J. Nat. Hist., 30: 505-521.

Guerao, G, Abelló, P. and Cuesta, J.A. - 1997. Morphology of the megalopa and first crab stage of the mediolittoral crab Pachygrapsus marmoratus (Brachyura, Grapsidae, Grapsinae). Zoosystema, 19(2-3): 437-447.

Guerao, G., Abelló, P. and Torres, P. - 1998. Morphology of the first zoea of the spider crab Macropodia linaresi (Brachyura, Majidae, Inachinae). Boll. Soc. Hist. Nat. Balears, 41: 13-18.

Guerao, G., Abelló, P. and Torres, P. - 1999. Morphology of the first zoea of the shamefaced crab Calappa granulata (Linnaeus, 1758) (Brachyura, Calappidae) obtained in the laboratory. Graellsia, 55: 157-162.

Gurney, A.R. - 1982. The larval development of Crangon crangon (Fabricius, 1795) (Crustacea, Decapoda). Bull. Br. Mus. Nat. Hist., 42(4): 247-262.

Gurney, R. - 1902. The metamorphosis of Corystes cassivelaunus (Penannt). Quart. J. Micr. Sci., 46: 461-478.

Gurney, R. - 1903. The larvae of certain british Crangonidae. $J$. Mar. Biol. Assoc. U. K., 6: 595-597.

Gurney, R. - 1903a. The metamorphosis of the decapod crustaceans Aegeon (crangon) fasciatus Risso and Aegeon (crangon) trispinosus Hailstone. Proc. Zool. Soc. London, 1903: 24-30.

Gurney, R. - 1923. The larval stages of Processa canaliculata Leach. J. Mar. Biol. Assoc. U. K., 13: 245-265.

Gurney, R. - 1924. The zoea of Eurynome aspera. J. Mar. Biol. Assoc. U. K., 13(2): 433-436.

Gurney, R. - 1924a. British Antarctic "Terra Nova" Expedition, 1910. Crustacea. Part IX. Decapoda larvae. Nat. Hist. Rep., 8(2): $37-202$.

Gurney, R. - 1924b. The larval development of some british prawns (Palaemonidae). I. Palaemonetes varians. Proc. Zool. Soc. London, 1924: 297-328.

Gurney, R. - 1924c. The larval development of some British prawns (Palaemonidae). II. Leander longirostris and Leander squilla. Proc. Zool. Soc. London, 1924: 961-982.

Gurney, R. - 1926. The protozoeal stage in Decapod development. Ann. Mag. Nat. Hist., 18(9): 19-27.

Gurney, R. - 1927. Report on the larvae of the Crustacea Decapoda: Suez Canal Expedition. Trans. Zool. Soc. London, 22(2): 231-286.

Gurney, R. - 1936. Larvae of Decapod Crustacea, I-III. Discovery Rep., 12: 377-440.

Gurney, R. - 1936a. Notes on some decapod Crustacea of Bermuda. II. The species of Hippolyte and their larvae. Proc. Zool. Soc. London, 1936: 25-32.

Gurney, R. - 1937. Larvae of Decapod Crustacea. IV. Hippolytidae. Discovery Rep., 14: 351-404.

Gurney, R. - 1937a. Notes on some decapod Crustacea from the Red Sea. I. The genus Processa. Proc. Zool. Soc. London, 107: 85-101.

Gurney, R. - 1938. The larvae of the Decapod Crustacea. Palaemonidae and Alpheidae. Sci. Rep. Great Barrier Reef Exp. (1928-1929), 6(1): 1-60.

Gurney, R. - 1939. Bibliography of the larvae of Decapod Crustacea. The Ray Society of London, London, $123 \mathrm{pp}$.

Gurney, R. - 1942. Larvae of Decapod Crustacea. The Ray Society of London, London, $306 \mathrm{pp}$.

Gurney, R. - 1943. The larval development of two penaeid prawns from Bermuda of the genera Sicyonia and Penaeopsis. Proc. Zool. Soc. London, 113: 1-16.

Gurney, R. and Lebour, M.V. - 1940. The larvae of decapod Crustacea. VI. The genus Sergestes. Discovery Rep., 20: 1-68.

Gurney, R. and Lebour, M.V. - 1941. On the larvae of certain Crustacea Macrura, mainly from Bermuda. J. Linn. Soc. London, 41: 89-181. 
Hansen, H.J. - 1922. Crustacés Décapodes (Sergestides) provenant des campagnes des campagnes des Yachts "Hirondelle" et "Princesse Alice" (1885-1915). Res. Camp. Sci. Monaco, 64: 1-232.

Hashizume, K. - 1999. Larval development of seven species of Lucifer (Dendrobranchiata, Sergestoidea) with a key for the identification of their larval forms. In: Schram, F.R. and Klein, von V. (eds). Crustaceans and Biodiversity Crisis: Proceedings of the Fourth International Crustaceans Congress, pp. 753799. Amsterdam.

Heegaard, P. - 1963. Decapod larvae from the Gulf of Napoli hatched in captivity. Vid. Medd. Dansk. Nat. For., 125: 449-493.

Heegaard, P. - 1966. Larvae of decapod Crustacea. The oceanic penaeids Solenocera, Cerataspis, Cerataspides. Dana Rep., 67: $1-147$.

Heegaard, P. - 1969. The first larval stages of Chlorotocus crassicornis (Decapoda, Pandalidae). Crustaceana, 17: 151-158.

Heldt, J.H. - 1938. La réproduction chez les Crustacés Décapodes de la famille des Pénéides. Ann. Inst. Oceanogr. Monaco, 18(2): 31-206

Heldt, J.H. - 1954. Stades larvaires d'Aristeomorpha foliacea (Risso) et Aristeus antennatus (Risso), Décapodes Penéides. $C$. R. Acad. Sci., 239: 1080-1082.

Heldt, J.H. - 1955. Contribution a l'étude de la biologie des Crevettes Pénéidés. Formes larvaires de Solenocera membranacea (Milne Edwards). Bull. Sta. Oceanogr. Salambo, 51: 29-56

Heldt, J.H. - 1955a. Contribution à l'étude de la biologie des crevettes Pénéides Aristaeomorpha foliacea (Risso) et Aristeus antennatus (Risso) (formes larvaires). Bull. Soc. des Sc. Nat. Tunisie, VIII, 1-2: 1-29.

Herring, P.J. - 1967. Observations on the early larvae of three species of Acanthephyra (Crustacea, Decapoda, Caridea). Deep Sea Res., 14: 325-329.

Hesse, M. - 1876. Descriptions des Crustacées rares ou nouveaux des côtes de France. Ann. Sci. Nat. Zool., 3(5): 1-42.

Hesse, M. - 1884. Crustacés rares ou nouveaux des côtes de France. Ann. Sci. Nat. Zool., 6: 1-14.

Hoglund, H. - 1943. On the biology and larval development of Leander squilla (L.) forma typica De Man. Svens. Hydr. Biol. Kommn., 6.: 1-44.

Hong, S.Y. - 1988. Development of epipods and gills in some pagurids and brachyurans. J. Nat. Hist., 22: 1005-1040.

Hong, S.Y. and Ingle, R.W. - 1987. Larval development of the circular crab, Atelecyclus rotundatus (Olivi) (Crustacea, Brachyura, Atelecyclidae) reared in the laboratory. J. Nat. Hist., 21: $1539-1560$.

Hood, M.R. - 1962. Studies on the larval development of Rithropanopeus harrissii (Gould) of the family Xanthidae (Brachyura). Gulf Res. Rep., 1(3): 122-130.

Hudinaga, M. - 1942. Reproduction, development and rearing of Penaeus japonicus, Bate. Jap. J. Zool., 10: 305-393.

Huus, J. - 1935. Zur morphologisch-systematische und biologischen kenntnis der nordischen Munida-larven (Crustacea, Decapoda). Bergens Mus. Aarb., 1934 (8): 1-29.

Hyman, O.W. - 1924. Studies on larvae of crabs of the family Grapsidae. Proc. U. S. Nat. Mus., 65(10): 1-8.

Hyman, O.W. - 1924a. Studies on the larvae of crabs of the family Pinnotheridae. Proc. U. S. Nat. Mus., 64(7): 1-9.

Illig, G. - 1914. Die Dekapoden des deutschen Süspolar Expediton. II: Die Sergestidae. Deuts. Sündp. Exp., 15: 347-376.

Illig, G. - 1927. Die Sergestiden. Wiss. Ergeb. Deuts Tiefsee Exp., 23: $279-354$

Ingle, R.W. - 1977. The larval and postlarval development of the Scorpion Spider Crab, Inachus dorsettensis (Pennant) (Family Majidae), reared in the laboratory. Bull. Br. Mus. Nat. Hist., 30: 329-348.

Ingle, R.W. - 1979. The larval and postlarval development of the brachyuran crab Geryon tridens Kröyer (Family Geryonidae) reared in the laboratory. Bull. Br. Mus. Nat. Hist., 36: 217-232

Ingle, R.W. - 1979a. The larval development of the spider crab Rochinia carpenteri (Thomson) (Oxyrhyncha, Majidae) with a review of majid subfamily larval features. Bull. Br. Mus. Nat. Hist., 37: 47-66.

Ingle, R.W. - 1981. The larval and postlarval development of the edible crab Cancer pagurus Linnaeus (Decapoda, Brachyura). Bull. Br. Mus. Nat. Hist., 40: 211-236.

Ingle, R.W. - 1982. Larval and post-larval development of the slen- der-legged spider crab, Macropodia rostrata (Linnaeus) (Oxyrhyncha, Majidae, Inachinae), reared in the laboratory. Bull. Br. Mus. Nat. Hist., 42: 207-225

Ingle, R.W. - 1983. A comparative study of the larval development of Monodaeus couchi (Couch), Xantho incisus (Leach) and Pilumnus hirtellus (Linnaeus) (Crustacea, Brachyura, Xanthidae). J. Nat. Hist. 17: 951-978.

Ingle, R.W. - 1984. The larval and post-larval development of the thumb-nail crab, Thia scutellata (Fabricius) (Decapoda, Brachyura). Bull. Br. Mus. Nat. Hist., 47(1): 53-64.

Ingle, R.W. - 1985a. Larval development of the red swimming crab, Bathynectes longipes (Risso, 1816) (Crustacea, Decapoda, Portunidae). Bull. Br. Mus. Nat. Hist., 49: 239-255.

Ingle, R.W. - 1987. The first zoea of three Pachygrapsus species and of Cataleptodius floridanus (Gibbes) from Bermuda and Mediterranean (Crustacea, Decapoda, Brachyura). Bull. Br. Mus. Nat. Hist., 52(1): 31-41.

Ingle, R.W. - 1990. Larval and post-larval development of Anapagurus chiroacanthus (Lilljeborg, 1855) (Anomura, Paguroidea, Paguridae). Bull. Br. Mus. Nat. Hist.,, 56(2): 105-134.

Ingle, R.W. - 1992. Larval stages of northeastern atlantic crabs. An illustrated key. Chapman \& Hall, London, 363 pp.

Ingle, R.W. - 1993. Hermits crab of the northeastern Atlantic Ocean and Mediterranean Sea: an illustrated key. Chapman \& Hall, London, 495 pp.

Ingle, R.W. and Clark, P.F. - 1980. The larval and postlarval development of Gibbs's spider crab Pisa armata (Latreille) (Family Majidae, Subfamily Pisinae) reared in the laboratory. J. Nat. Hist., 14: 723-735.

Ingle, R.W. and Clark, P.F. - 1983. The larval development of the angular crab Goneplax rhomboides (Linnaeus) (Decapoda, Brachyura). Bull. Br. Mus. Nat. Hist., 44: 163-177.

Ingle, R.W. and Rice, A.L. - 1971. The larval development of the masked crab Corystes cassivelaunus (Pennant) (Brachyura, Corystidae) reared in the laboratory. Crustaceana, 20(3): 271-284.

Issel, R. - 1910. Ricerche intorno alla biologia ed alla morfologia dei crostacei decapodi. Parte I: Studi sui Paguridi. Arc. Zool., 4(3): 335-397.

Jorgensen, O.M. - 1918. Notes on development of Carcinus maenas. Rep. Dove Mar. Lab., 7: 62-64.

Jorgensen, O.M. - 1925. Some crustaceans larvae from the Northumberland plankton. Trans. Nat. Hist. Soc. Northumb., 6: 157-166.

Jorgensen, O.M. - 1925a. The early stages of Nephrops norvegicus from the Northumberland plankton, together with a note on the post-larval Homarus vulgaris. J. Mar. Biol. Ass. U. K., 13: $870-876$.

Kaya, B. and Özel, I. - 1992. Planktonic larval stages of porcellanid Crustacea (Crustacea, Decapoda, Anomura) in Izmir Bay. $J$. Fac. Sci. Ege Univ., B, 14(2): 79-91.

Kemp, S.W. - 1907. Biscayan plankton. XI Decapoda. Trans. Linn. Soc. London, 10: 205-217.

Kemp, S.W. - 1910. The Decapoda Natantia of the coast of Ireland. Fish. Ire. Sci. Inv. 1908, 1: 1-190.

Kim, K.B. and Hong, S.Y. - 1999. Larval development of the wrinkled swimming crab Liocarcinus corrugatus (Decapoda, Brachyura, Portunidae) reared in the laboratory. J. Crust. Biol., 19(4): 792-808

Kinahan, J.R. - 1860. Notes on the foregoing paper, with a supplement to his list of Dublin Crustacea. Proc. Nat. Hist. Soc. Dublin, 2: 43-51.

Knowlton, R.E. and Alavi, M.R. - 1995. The larval morphology of Lysmata anchisteus Chace (Crustacea, Decapoda) compared with other Lysmata spp. Can. J. Sci., 31: 289-310.

Kurata, H. - 1968. Larvae of Decapoda Anomura of Arasaki, Sagami Bay. II. Dardanus arrosor (Herbst) (Diogenidae). Bull. Tokai Reg. Fish. Res. Lab., 56: 173-180.

Kurian, C.V. - 1956. Larvae of decapod Crustacea from the Adriatic Sea. Acta Adriatica, 6(3): 1-108.

Latreille, P.A. - 1802. Historie Naturelle, Générale et Particulère des Crustacés et des Insectes. Paris, $387 \mathrm{pp}$.

Le Roux, A. - 1963. Contribution a l'étude du développement larvaire d'Hippolyte inermis Leach (Crustacé Décapode Macroure). C. R. Hebd. Sean. Acad. Sci., 256: 3499-3501.

Le Roux, A. - 1966. Le développement larvaire de Porcellana longicornis Pennant (Crustacé, Décapode, Anomoure, Galatheide). Cah. Biol. Mar., 7: 69-78. 
Le Roux, A. - 1966a. Contribution a l'étude du développement larvaire de Clibanarius erythropus (Latreille) (Crustacé, Décapode, Anomure, Diogénidé). Cah. Biol. Mar, 7: 225-230.

Leach, W.E. - 1817. The zoological Miscellany, Being Descriptions of New or Interesting Animals. London, $151 \mathrm{pp}$.

Lebour, M.V. - 1924. Larval crabs from Bermuda. Rep. Zool. Sci. Cont., 29: 113-128.

Lebour, M.V. - 1925. The eggs and newly hatched larvae of Typton spongicola Costa. J. Mar. Biol. Ass. U. K., 13(4): 848-853.

Lebour, M.V. - 1927. Studies of the Plymouth Brachyura. I. The rearing of the crabs in captivity with a description of the larval stages of Inachus dorsettensis, Macropodia longirostris and Maia squinado. J. Mar. Biol. Ass.. U. K., 14(3): 795-821.

Lebour, M.V. - 1928. The larval stages of the Plymouth Brachyura. Proc. Zool. Soc. London, 1928: 473-560.

Lebour, M.V. - 1928a. Studies of the Plymouth Brachyura. II. The larval stages of Ebalia and Pinnotheres. J. Mar. Biol. Ass.. U. K., 15: 109-122.

Lebour, M.V. - 1930. The larvae of the Plymouth Galatheidae. I. Munida banffica, Galathea strigosa and Galathea dispersa. J. Mar. Biol. Ass. U. K., 17: 175-184.

Lebour, M.V. - 1931. The larvae of the Plymouth Caridea. I The larvae of the Crangonidae. II The larvae of the Hippolytidae. Proc. Zool. Soc. London, 1931: 1-9.

Lebour, M.V. - 1931a. The larvae of the Plymouth Galatheidae. II. Galathea squamifera and Galathea intermedia. J. Mar. Biol. Ass. U. K., 17: 385-390.

Lebour, M.V. - 1931b. Further notes on larval Brachyura. Proc. Zool. Soc. London, 1: 93-96.

Lebour, M.V. - 1932. The larval stages of the Plymouth Caridea III: The larval stages of Spirontocari cranchii (Leach). Proc. Zool. Soc. London, 1932: 131-137.

Lebour, M.V. - 1932a. The larval stages of the Plymouth Caridea IV: The Alpheidae. Proc. Zool. Soc. London, 1932: 463-469.

Lebour, M.V. - 1933. Rearing marine animals in a plunger jar. Sci. Prog., 107: 494-502.

Lebour, M.V. - 1934a. The life history of Dromia vulgaris. Proc. Zool. Soc. London, 2: 241-249.

Lebour, M.V. - 1936. Notes on the Plymouth Species of Spirotoncaris (Crustacea). Proc. Zool. Soc. London, 1936: 89-104.

Lebour, M.V. - 1936a. Notes on the Plymouth Processa (Crustacea). Proc. Zool. Soc. London, 1936: 609-617.

Lebour, M.V. - 1940. The larvae of the Pandalidae. J. Mar. Biol. Ass. U. K., 24: 239-252

Lebour, M.V. - 1941. Notes on Thalassinid and Processid larvae (Crustacea, Decapoda) from Bermuda. Ann. Mag. Nat. Hist., 7: 401-420.

Lebour, M.V. - 1943. The larvae of the genus Porcellana (Crustacea, Decapoda) and related forms. J. Mar. Biol. Ass. U. K., 25: 721-737.

Lebour, M.V. - 1944. The larval stages of Portumnus (Crustacea, Brachyura) with notes on some other genera. J. Mar. Biol. Ass. U. K., 26: 7-15.

Lebour, M.V. - 1944a. Larval crabs from Bermuda. Zoologica, 29(11): 113-128.

Lebour, M.V. - 1949. The last larva and postlarva of Typton spongicola from Plymouth (Crustacea, Decapoda). J. Mar. Biol. Ass. U. K., 28: 667-672.

Lebour, M.V. - 1959. The larval decapod Crustacea of Tropical West Africa. Atlant. Rep., 5: 119-143.

Legendre, R. - 1940. La faune pélagique de L'Atlantique au large du Golfe de Gascogne, récueillie dans des estomacs de Germons. Troisième partie: invertébrés (cephalopodes exclus) Parasites du Germon. Ann. Inst. Oceanogr., 20: 127-310.

Linnaeus, C. - 1767. Systema naturae per Regna Tria Naturae, Secundum Classes, Ordines, Genera, Species, cum characteribus, differentiis, synonymus, locis. Ed. 12, Pt 2. Stockholm, $1047 \mathrm{pp}$.

Lo Bianco, S. - 1901. Le pesche pelagiche abissali eseguite dal "Maia" nelle vicinanze di Capri. Mitt. Zool. Sta. Neapel, 15. 413-482.

Lo Bianco, S. - 1903. La pesche abissali eseguite da F.A. Krupp Col Yacth Puritan nelle adiacenze di Capri ed in altre località del mediterraneo. Mitt. Zool. Sta. Neapel, 16: 109-279.

Lo Bianco, S. - 1904. Pelagische Tiefseefischerei der "Maja" in der Umgebung von Capri. Beitr. Ken. Meer. Bewohner, 1: 1-91.

Lumare, F. and Gozzo, S. - 1972. Sviluppo larvale del crostaceo Xanthidae Eriphia verrucosa (Förskal, 1775) in condizioni di laboratorio. Boll. Pesca Pisc. Idrobiol., 27: 185-209.

Lumare, F. and Gozzo, S. - 1973. Observazioni sulla morfogenesi del nauplio de $P$. kerathurus nelle sue relazioni con $P$. japonicus. Atti Soc. Pelorit. Sci. Fis. Mat. Nat., 18: 97-116.

Lutze, J. - 1938. Ueber systematik, entwicklung und oekologie von Callianassa. Helgol. Wiss. Meeresunters. Ach., 1: 162-199.

MacDonald, J.D., Pike, R.B. and Williamson, D.I. - 1957. Larvae of the British species of Diogenes, Pagurus, Anapagurus and Lithodes (Crustacea, Decapoda). Proc. Zool. Soc. London, 128: 209-257.

Makarov, R.R. and Golodetsky, L.A. - 1980. Larval development of the Black Sea prawn Palaemon adspersus (Crustacea, Decapoda) in the laboratory. Zool. Zh., 59(3): 375-382.

Martin, J.W. - 1984. Notes and bibliography on the larvae of Xanthid crabs, with a key to the known xanthid zoeas of the western atlantic and gulf of Mexico. Bull. Mar. Sci., 34: 220-239.

Mayer, P. - 1877. Zur Entwicklungsgeschichte der Dekapoden. Jena. Z. Natur., 11: 188-269.

Mayer, P. - 1878. Uber einige Jugendstadien von Penaeus caramote. Mitt. Zool. Sta. Neapel, 1: 49-51.

Mene, L.J. - 1987. Aspectos morfológicos de la fases larvarias de la nécora, Liocarcinus puber. Bol. Inst. Esp. Oceanogr., 4(1): 61-68.

Menon, M.K. - 1937. Decapod larvae from the Madras plankton. Bull. Madras Gov. Mus., 3: 1-55.

Miers, E.J. - 1881. Account of the zoological collections made during the survey of H.M.S. "Alert" in the Strait of Magellan and on the coast of Patagonia. Crustacea. Proc. Zool. Soc. London, 1881: 61-80.

Montagu, G. - 1804. Description of several marine animals found on the south coast of Devonshire. Trans. Linn. Soc. London, 7: 61-85.

Monticelli, F.S. and Lo Bianco, S. - 1900. Sullo sviluppo dei Penaeidae nel golfo di Napoli. Monit. Zool. Ital., 11: 23-31.

Monticelli, F.S. and Lo Bianco, S. - 1901. Uova e larva di Solenocera siphonocera. Monit. Zool. Ital., 12: 205-206.

Monticelli, F.S. and Lo Bianco, S. - 1902. Su la probabile larva di Aristeus antennatus, Risso. Monit. Zool. Ital., 13: 30-31.

Mortensen, T. - 1897. Undersögelser over vor almindelige Rejes (Palaemon fabricii Rathke). Biologi og Udviklingshistorie samt Bemaerkninger om Rejesfiskeriet i de Danske farvande. Vid. Undersög. Fisk. omr. Udg. Dansk Fiskerifor., 1: 1-79.

Müller, F. - 1862. On the transformations of the Porcellanae. Ann. Mag. Nat. Hist., ser. 3, 11:47-50.

Mura, M. and Pessani, D. - 1994. Descrizione del primo stadio larvale e notizie sul periodo riproducttivo di alcune specie di decapodi. Biol. Mar. Medit., 1(1): 391-392.

Neumann, V. - 1993. The morphology of the first zoea stage of the spider crab Maja crispata (Risso, 1827). Senckenberg. Mar., 23(4/6): 143-148

Ngoc-Ho, N. - 1981. A taxonomic study of the larvae of four thalassinid species (Decapoda, Thalassinidea) from the gulf of Mexico. Bull. Brit. Mus. Nat. Hist., 40: 237-273.

Nichols, J.H. and Lawton, P. -1978 . The occurrence of the larval stages of the lobster Homarus gammarus, (Linnaeus, 1758) off the northeast coast of England in 1976. Cons. Int. Explor. Mer, 38(2): 234-243

Nöel, P.Y. - 1992. Clé preliminaire d'identification des Crustacea Decapoda de France et des principales autres espèces d'Europe. Collection Patrimoines Naturalles. Muséum National d'Historie Naturels Paris. Paris, 145 pp.

Ortmann, A. - 1893. Decapoden und Schizopoden. Plankton Expedition der Humboldt Stiftung. Wiss. Ergebn., 2: 1-120.

Paula, J. - 1985. The first zoeal stages of Polybius henslowi Leach, Maja squinado (Fabricius), and Uca tangeri (Eydoux) (Crustacea, Decapoda, Brachyura). Arq. Mus. Boc., B2(17): 137-147.

Paula, J. - 1987. Planktonic stages of brachyuran crabs from the south-western Iberian coast (Crustacea, Decapoda, Brachyura). J. Nat. Hist., 21: 717-756.

Paula, J. - 1988. The larval development of the spider crab Maja goltziana d'Oliveira, 1888 (Crustacea, Decapoda, Majidae) reared in the laboratory. J. Nat. Hist., 22: 1697-1708.

Paula, J. - 1988a. The larval and post-larval development of Pennant's swimming crab, Portumnus latipes (Pennant) (Brachyura, Portunidae) reared in the laboratory. Crustaceana, 55(2): 202-216

Paula, J. - 1991. The zoeal stages of the crab Medorippe lanata (Linnaeus, 1767) (Brachyura, Dorppidae) reared in the labora- 
tory, and the larval characters of the Dorippidae. J. Nat. Hist., 25: $75-89$

Paula, J. and Hartnoll, R.G. - 1989. The larval and post-larval development of Percnon gibbesi (Crustacea, Brachyura, Grapsidae) and the identity of the larval genus Pluteocaris. J. Nat. Hist., 21: 17-37.

Paulinose, V.T. - 1984. Larval and postlarval stages of the genus Trachypenaeopsis Burkenroad (Penaeidae, Penaeinae) from the Indian Ocean. Bull. Natn. Inst. Oceanogr. Mahasagar, 17: 221-226.

Pearson, J.C. - 1908. Cancer (The edible Crab). Proc. Trans. Liverpool Biol. Soc., 22: 291-499.

Pérez-Farfante, I. and Kensley, B. - 1997. Penaeoid and Sergestoid shrimps and prawns of the world - Keys and diagnoses for the families and genera. Mem. Mus. natn. Hist. nat., 175: 1-233.

Pessani, D. - 1993. Planktonic larvae of Natantia (Decapoda) in Tigullio Gulf (Ligurian Sea), with remarks on larvae of Pandalidae. Crustaceana, 65(3):309-317.

Pessani, D. and Godino, C. - 1991. Larval development of Philocheras trispinosus (Hailstone, 1835) (Decapoda, Crangonidae) reared in the laboratory. J. Crust. Biol., 11: 123-137.

Pessani, D. and Robotti, C.A. - 1992. Larvae of the mediterranean decapod crustaceans: summary of the literature until 1988. Oebalia, 17: 387-397.

Pike, R.B. and Williamson, D.I. - 1958. Crustacea Decapoda: Larvae. XI. Paguridea, Coenobitidae, Dromiidea, and Homolidea. Fich. Ident. Zoopl., 81: 1-9.

Pike, R.B. and Williamson, D.I. - 1960. Larvae of decapod Crustacea of the families Diogenidae and Paguridae from the bay of Naples. Publ. Staz. Zool. Nap., 31: 493-552.

Pike, R.B. and Williamson, D.I. - 1960a. Larvae of decapod Crustacea of the families Dromiidae and Homolidae from the bay of Naples. Publ. Staz. Zool. Nap., 31: 553-563.

Pike, R.B. and Williamson, D.I. - 1961. Larval variation in Philocheras bispinosus (Hailstone) (Decapoda, Crangonidae). Crustaceana, 2: 21-25.

Pike, R.B. and Williamson, D.I. - 1961a. The larvae of Spirontocaris and related genera (Decapoda, Hippolytidae). Crustaceana, 2: 187-208.

Pike, R.B. and Williamson, D.I. - 1964. The larvae of some species of Pandalidae (Decapoda). Crustaceana, 6: 265-284.

Pike, R.B. and Williamson, D.I. - 1972. Crustacea Decapoda: Larvae. X. Galatheidea. Fich. Ident. Zoopl., 139: 1-5.

Ramonell, R. - 1987. Estudio morfológico de los estadios larvarios del camarón Palaemon serratus (Pennant, 1977). Inv. Pesq., 51 (Supl.1): 545-560.

Rathbun, M.J. - 1923. The brachyuran crabs collected by the U.S. Fisheries Steamer "Albatros" in 1911, chiefly on the west coast of Mexico. Bull. Amer. Mus. Nat. Hist., 48:619-637.

Rice, A.L. - 1964. The metamorphosis of a species of Homola (Crustacea, Decapoda, Dromiacea). Bull. Mar. Sci. Gulf Caribb., 14: 221-238.

Rice, A.L. - 1975. The first zoeal stages of Cancer pagurus L., Pinnotheres pisum (Pennant) and Macrophthalmus depressus Ruppel (Crustacea, Decapoda, Brachyura). Bull. Br. Mus. Nat. Hist., 28: 237-247.

Rice, A.L. - 1980. Crab zoeal morphology and its bearing on the classification of the Brachyura. Trans. Zool. Soc. London, 35: 271-424.

Rice, A.L. - 1980a. The first zoeal stage of Ebalia nux Milne Edwards, 1883, with a discussion of the zoeal characters of the Leucosiidae (Crustacea, Decapoda, Brachyura). J. Nat. Hist., 14: 331-337.

Rice, A.L. - 1982. The megalopa stage of Latreillia elegans Roux, 1830 (Decapoda, Brachyura, Homoloidea). Crustaceana, 43(2): 205-210.

Rice, A.L. and Ingle, R.W. - 1975. The larval development of Carcinus maenas (Linnaeus) and Carcinus mediterraneus Czerniavsky (Crustacea, Brachyura, Portunidae) reared in the laboratory. Bull. Br. Mus. Nat. Hist., 28(3): 101-119.

Rice, A.L. and Ingle, R.W. - 1975a. A comparative study of the larval morphology of the British portunid crabs Macropipus puber (L.) and Macropipus holsatus (Fabricius), with a discussion of generic and subfamily larval characters within the Portunidae. Bull. Br. Mus. Nat. Hist., 28: 121-151.

Rice, A.L. and Ingle, R.W. - 1978. The larval development of the portunid crab Macropipus pusillus (Leach) reared in the laboratory. Bull. Br. Mus. Nat. Hist., 33: 287-296.

Rice, A.L., Ingle, R.W. and Allen, E. - 1970. The larval develop- ment of the sponge crab Dromia personata (L.) (Crustacea, Decapoda, Brachyura) reared in the laboratory. Vie et Milieu, 21: $223-240$.

Rice, A.L. and Provenzano, A.J. - 1966. The larval development of the West Indian sponge crab Dromidia antillensis (Decapoda, Dromiidae). J. Zool. London, 149: 297-319.

Rice, A.L. and Provenzano, A.J. - 1970. The larval stages of Homola barbata (Fabricius) (Crustacea, Decapoda, Homolidae) reared in the laboratory. Bull. Mar. Sci., 20: 446-471.

Rice, A.L. and Von Levetzon, K.G. - 1967. Larvae of Homola (Crustacea, Dromiacea) from South Africa. J. Nat. Hist., 1: 435-453.

Rice, A.L. and Williamson, D.I. - 1977. Planktonic stages of Crustacea Malacostraca from the Atlantic Sea Mounts. Meteors Fors., (D) 26: 28-64.

Roberts, M.H. - 1969. Larval development of Bathynectes superba (Costa) reared in the laboratory. Biol. Bull., 137:338-351.

Rochanaburanon, T. and Williamson, D.I. - 1976. Laboratory survival of Palaemon elegans Rathke and other caridean shrimps in relation to their distribution and ecology. Estuar. Coast. Mar. Sci., 4: 83-91.

Rodríguez, A. - 1997. Larval and postlarval development of Pisa tetraodon (Pennant, 1777) (Decapoda, Majidae) reared in the laboratory. J. Plank. Res., 19(1): 29-41.

Rodríguez, A., González-Gordillo, J.I. and Cuesta, J.A. - 1992. Larval stages of Brachynotus atlanticus Forest, 1957 (Crustacea, Decapoda, Grapsidae) reared under laboratory conditions. J. Plank. Res., 14(6): 867-883.

Rodríguez, A. and Jones, D.A. - 1993. Larval development of Uca tangeri (Eydoux, 1835) (Decapoda, Ocypodidae) reared in the laboratory. J. Crust. Biol., 13(2): 309-321.

Rodríguez, A. and Martin, J.W. - 1997. Larval development of the crab Xantho poressa (Decapoda, Xanthidae) reared in the laboratory. J.Crust.Biol., 17(1):98-110.

Rodríguez, A. and Paula, J. - 1993a. Larval and postlarval development of the mud crab Panopeus africanus Milne Edwards (Decapoda, Xanthidae) reared in the laboratory. J. Crust. Biol., 13(2): 296-308

Roff, J.C., Davidson, K.G., Pohle, G. and Dadswell, M.J. - 1984. A guide to the marine flora and fauna of Bay of Fundy and Scotian Shelf: larval Decapoda: Brachyura. Can. Tech. Rep. Fish. Aquat. Sci., 132: 1-57.

Rossignol, M.-1957. Crustacés Décapodes Marines de la Region de Pointe-Noire. In: J. Collignon, M. Rosignol, and Ch. Roux (eds.): Mollusques, Crustacés, Poissons Marines des côtes d'AEF en collection au centre d'Oceanographie de l'Institut d'Etudes Centrafricaines de Pointe Noire, pp. 71-136. Paris.

Saint Laurent - Dechancé, M. - 1964. Dévelopment et position systématique du genre Parapagurus Smith (Crustacea, Decapoda, Paguridea). I. Description des states larvaires. Bull. Inst. Oceanogr. Monaco, 64(1321): 1-26.

Salman, S.D. - 1981. Larval development of Macropodia tenuirostris (Leach) (Crustacea, Brachyura, Majidae) reared in the laboratory. J. Nat. Hist., 15: 931-938.

Salman, S.D. - 1982. Larval development of the spider crab Eurynome aspera (Pennant) reared in the laboratory with a key to the known larvae of the subfamily Pisinae (Brachyura, Majidae). Crustaceana, 43(1): 78-88.

Salman, S.D. - 1982a. Observations on the larvae of north european crabs of the genus Ebalia (Brachyura, Leucosiidae). Crustaceana, 42:256-269.

Salman, S.D. - 1982b. Larval development of the crab Pilumnus hirtellus (L.) reared in the laboratory (Decapoda, Brachyura, Xanthidae). Crustaceana, 42(2): 113-126.

Samuelsen, T.J. - 1972. Larvae of Munidopsis tridentata (Esmark) (Decapoda, Anomura) reared in the laboratory. Sarsia, 48: 91-98.

Samuelsen, T.J. - 1972a. Larvae of Pagurus variabilis MilneEdwards and Bouvier (Decapoda, Anomura) reared in the laboratory. Sarsia, 48: 2-11

Samuelsen, T.J. - 1976. The first zoea of Paromola cuvieri (Risso) (Decapoda, Homolidae). Sarsia, 62: 5-8.

Sankarankutty, C. - 1968. Larvae of an unrecorded pagurid (Crustacea, Paguridae) from western Norway. Sarsia, 31: 57-62.

Santos, A. dos and Lindley, J.A. - In press. Crustacea Decapoda: Larvae II. Dendobranchiata (Aristeidae, Benthesicymidae, Penaeidae, Solnoceridae, Sicyonidae, Sergestidae and Luciferidae). Fich. Ident. Zoopl., 30. 
Santucci, R. - 1925. Contributo allo studio dello sviluppo post embrionali degli Scyllaridea del Mediterraneo I Palinurus vulgaris. Atti Accad. naz. Lincei Rc., 6: 333-338.

Santucci, R. - 1925a. Contributo allo studio dello sviluppo postembrionale degli Scyllaridea del Mediterraneo II: Scyllarus arctus. III: Scyllarides latus. Mem. R. Com. Talassogr. Ital., 71: $1-16$.

Santucci, R. - 1926. Lo Sviluppo e l'ecologia Postembrionali dello Scampo Nephrops norvegicus (L.) nel Tirreno e nei mari nordici. Mem. R. Com. Talassogr. Ital., 125: 1-36.

Santucci, R. - 1926a. Fillosomi di Scillaridi esotici nel mediterraneo. Monit. Zool. Ital., 27: 19-23.

Santucci, R. - 1926b. Lo stadio natante e la prima forma postnatante dell'Aragosta (Palinurus vulgaris Latr.) del Mediterraneo. Mem. R. Com. Talassogr. Ital., 127: 1-11.

Santucci, R. - 1927. Uno stadio sviluppo non ancora descritto dello "Scampo" Nephrops norvegicus (L.) Mem. R. Com. Talassogr. Ital., 128: 1-6.

Santucci, R. - 1928. Il primo stadio post-embrionale di Scyllarides latus Latreille. Mem. R. Com. Talassogr. Ital., 144: 1-7.

Sars, G.O. - 1875. Om hummerens postembryonale udvikling. Forh. Vidensk. Selsk. Krist., 1874: 1-27.

Sars, G.O. - 1884. Bidrag til kundskaben om decapodernes forvandlinger. I. Nephrops, Calocaris, Gebia. Arc. Math. Natur., 9: 155-204.

Sars, G.O. - 1889. Bidrag til kundskaben om decapodernes forvandlinger. II. Lithodes, Eupagurus, Spiropagurus, Galathodes, Galathea, Munida, Porcellana, Nephrops. Arc. Math. Natur., 13: 133-201.

Sars, G.O. - 1890. Bidrag til kundskaben om Decapodernes Forvandlinger. III: Crangonidae. Arc. Math. Natur. 14: 132-195.

Sars, G.O. - 1900. Account of the postembryonal development of Pandalus borealis, with remarks on the development of other pandali, and description of the adult Pandalus borealis. Rep. Norw. Fish. Mar. Inv., 1(3): 1-45.

Sars, G.O. - 1906. Postembryonal development of Athanas nitescens Leach. Arc. Math. Natur., 27(10): 1-29.

Sars, G.O. - 1911. Account of the post-embryonal development of Hippolyte varians Leach. Arc. Math. Natur., 32(7): 1-25.

Schlegel, C. - 1911. Sur le developpement de Maia squinado Latr. C. R. Hebd. Seanc. Acad. Sci., 153(8): 480-482.

Sekiguchi, H. - 1986. Identification of late-stage phyllosoma larvae of the scyllarid and palinurid lobsters in the japanese waters. Bull. Jap. Soc. Sci.Fish., 52(8): 1289-1294.

Selbie, C.M. - 1914. The Decapoda Reptantia of the coast of Ireland. I. Palinura, Astacura and Anomura (except Paguridea) Sci. Invest. Fish. Br. Ireland, 1: 1-116.

Seridji, R. - 1971. Contribution a l'étude des larves crustacés décapodes en baie d'Alger. Pelagos, 3(2): 1-107.

Seridji, R. - 1988. Some planktonic larval stages of Albunea carabus (L., 1758) (Crustacea, Decapoda, Anomura). J. Nat. Hist., 22: 1293-1300.

Seridji, R. - 1989. Etude des larves des crustacés décapodes. aspects taxonomique, écologique et biogeographique. Ph.D. thesis, Alger, $618 \mathrm{pp}$

Seridji, R. - 1990. Description of some planktonic larval stages of Stenopus spinosus Risso, 1826: notes on the genus and the systematic position of the Stenopodidea as revealed by larval characters. Sci. Mar., 54(3): 293-303.

Seridji, R. - 1993. Descriptions of some planktonic larvae of the Calappidae (Crustacea, Decapoda, Brachyura). J. Plank. Res. 15(4): 437-453.

Shen, C.J. - 1935. An investigation of the post-larval development of the shore-crab Carcinus maenas, with the special reference to the external secondary sexual characters. Proc. Zool. Soc. London, 1935: 1-33.

Sivertsen, E. and Holthuis, L.B. - 1956. Crustacea Decapoda (The Penaeidea and Stenopodidea excepted). Rep. Sci. Res "Michael Sars" N. Atl. Exp., 1910: 121-154.

Slabber, M. - 1778. Natuurkundige Verlustigingen, behelzende microscopise waarneemingen van in-en uitlandse Water-en Land-Dieren. Haarlem, $166 \mathrm{pp}$.

Slabber, M. - 1781. Physikalische Belustigungen, order mikroskopische Wahrnehmungen von drey und vierzig inund ausländischen Wassere-und Landthierchen. Aus dem Holländischen übersezt. Nürnberg, $99 \mathrm{pp}$.

Sollaud, E. - 1912. Les Metamorphoses du Bocquet. C. R. Acad. Sci. Paris, 154: 664-667.
Sollaud, E. - 1923. Le developpement larvaire des Palamoninae. Bull. Biol. Fr.Belg., 57: 509-603.

Soltanpour-Gargari, A., Engelmann, R. and Wellershaus, S. - 1989. Development and rearing of zoea larvae in brachyura (Crustacea Decapoda). A bibliography. Crustaceana, Supl. 14: 1-173.

Stephensen, K. - 1916. Zoogeographical investigations of certain fjords in southern Greenland with special reference to the Crustacea, Pycnogonida and Echinodermata. Medd. Gronland, 53: 231-378.

Stephensen, K. - 1923. Decapoda Macrura (excl. Sergestidae). Rep. Danish Oceanogr.Exp. 1908-1910, 2: 1-85.

Sund, O. - 1920. Report of the Scientific Results of the "Michel Sars" North Atlant. Deep Sea Exp. 1910, 3(2): 1-32.

Sutton, A. H., Main, G. and Ronald, A.- 1969. An instrument for counting the larvae of the prawn Palaemon serratus and the brine shrimp Artemia salina. Lab. Pract., 18: 433-436.

Taishaku, H. and Konishi, K. - 1995. Zoeas of Calappa species with special reference to larval characters of the family Calappidae (Crustacea, Brachyura). Zool. Sci., 12: 649-654.

Tattersall, W.M. - 1938. A note on the trachelifer larva of Jaxea nocturna (Chiereghin) and its metamorphosis. Ann. Mag. Nat. Hist, ser. 11, 1(6): 625-631.

Terada, M. - 1987. Zoeal forms of 14 species of crabs from the Enshunada. Res. Crust., 16: 93-120.

Thiele, J. - 1905. Uber einige stieläugige Krebse von Messina. Zool. Jb., Supl. 8: 443-474.

Thiriot, A. - 1973. Stades larvaires de Parthenopidae méditerranées: Heterocrypta maltzani Miers et Parthenope massena (Milne Edwards). Cah. Biol. Mar., 14(2): 111-134.

Thiriot, A. - 1974. Larves de décapodes Macrura et Anomura, espèces européenes; caractères morphologiques et observations ècològiques. Thal. Jugosl., 10: 341-378.

Thompson, J.V. -1828 . On the metamorphoses of the Crustacea, and on the zoea, exposing their singular estructure, and demonstrating that they are not, as has been supposed, a peculiar genus, but the larva of Crustacea. Zool. Res. Illust. Nat. Hist., vol. I.: $1-11$.

Thompson, J.V. - 1835. Memoir on the metamorphosis in Porcellana and Portunus. Entom. Mag., 3: 275-280.

Thompson, J.V. - 1836. Memoir on the metamorphosis in the Macrourae or long-tailed Crustacea, exemplified in the prawn (Palaemon serratus). Edinburgh New Phil. J., 21: 221-223.

Tsurnamal, M. - 1963. Larval development of the prawn Palaemon elegans Rathke (Crustacea, Decapoda) from the coast of Israel. Israel J.Zool., 12: 117-141.

Wasserloos, E. - 1908. Zur kenntis der metamorphose von Sergestes arcticus. Kr. Zool. Anz., 33: 303-331.

Wear, R.G. - 1985. Checklist and annotated bibliography of New Zealand decapod crustacean larvae (Natantia, Macrura Reptantia, and Anomura). Zool. Publ. Victoria Univ. Wellington, 79: $1-15$

Wear, R.G. and Fielder, D.R. - 1985. The Marine Fauna of New Zealand: Larvae of the Brachyura (Crustacea, Decapoda). Mem. N. Z. Oceanogr. Inst., 92: 1-90.

Webb, G.E. - 1919. The development of the species of Upogebia from Plymouth Sound. J. Mar. Biol. Assoc. U. K., 12: 81-134.

Webb, G.E. - 1921. The larvae of the Decapoda Macrura and Anomura of Plymouth. J. Mar. Biol.Assoc. U. K., 12(3): 385-425.

Williams, B.G. - 1968. Laboratory rearing of the larval stages of Carcinus maenas (L.) (Crustacea: Decapoda). J. Nat. Hist., 2: 121-126.

Williamson, D.I. - 1957. Crustacea Decapoda: larvae. I. General. Fich.Ident. Zoopl., 67: 1-7.

Williamson, D.I. - 1957a. Crustacea Decapoda: Larvae. V. Caridea, Family Hippolytidae. Fich. Ident. Zoopl., 68: 1-5.

Williamson, D.I. - 1960. Crustacea Decapoda: larvae. VII. Caridea, Family Crangonidae; Stenopodidea. Fich. Ident. Zoopl., 90: 1-5.

Williamson, D.I. - 1960a. A remarkable zoea, attributed to the Majidae (Decapoda, Brachyura). Ann. Mag. Nat. Hist, 13(3): 141-144.

Williamson, D.I. - 1960b. Larval stages of Pasiphaea sivado and some other Pasiphaeidae (Decapoda). Crustaceana, 1(4): 331-341.

Williamson, D.I. - 1962. Crustacea Decapoda: larvae. III. Caridea, Families Oplophoridae, Nematocarcinidae, and Pasiphaeidae. Fich. Ident. Zoopl., 92: 1-5.

Williamson, D.I. - 1965. Some larval stages of three australian crabs belonging to the families Homolidae and Raninidae, and 
observations on the affinities of these families (Crustacea, Decapoda). Austr. J. Mar. Freshw. Res., 16: 369-398.

Williamson, D.I. - 1967. On a collection of planktonic Decapoda and Stomatopoda (Crustacea) from the Mediterranean coast of Israel. Bull. Sea Fish. Res. Sta. Haifa, 45: 32-64.

Williamson, D.I. - 1967a. Crustacea Decapoda: Larvae. IV. Caridea. Families: Pandalidae and Alpheidae. Fich. Ident. Zoopl., 109: 1-5.

Williamson, D.I. - 1970. On a collection of planktonic Decapoda and Stomatopoda (Crustacea) from the east coast of the Sinai Peninsula, Northen Red Sea. Bull. Sea Fish.Res. Sta. Haifa, 56: $3-48$

Williamson, D.I. - 1974. Larval characters and the origin of crabs (Crustacea, Decapoda, Brachyura). Thal. Jugosl., 10: 401-414.

Williamson, D.I. - 1982. The larval characters of Dorhynchus thomsoni Thomson (Crustacea, Brachyura, Majoidea) and their evolution. J. Nat. Hist., 16: 727-744.

Williamson, D.I. - 1983. Crustacea Decapoda: Larvae. VIII. Nephropidae, Palinuridea, Palinuridea, and Eryonidea. Fich. Ident. Zoopl., 167/168: 1-8.

Williamson, D.I. and Rochanaburanon, T. - 1979. A new species of Processidae (Crustacea, Decapoda, Caridea) and the larvae of the north European species. J. Nat. Hist., 13: 11-33.

Williamson, H.C. - 1910. Report on the larval and later stages of Portunus holsathus, Fabr.; Portunus puber, L.; Portunus depu-

\section{APPENDIX}

Synomyms or inaccurate names assigned to larval material (left column) and its corresponding correct name (right column). Current valid names are in italics; synonyms are in non italics.

Acanthephyra debilis Acanthephyra purpurea

Aegeon fasciatus Alpheus ruber

Amalopenaeus elegans

Anapagurus laevis

Aristaeomorpha foliacea

Aristeus antennatus

Astacus gammarus

Astacus norvegicus

Atelecyclus septemdentatus

Bathynectes superba

Calcinus ornatus

Callianassa stebbingi

Callianassa subterranea

Calliaxis adriatica

Calocaris macandreaea

Cancer germanus

Cancer rhomboidalis

Carcinus mediterraneus

Catapaguroides timidus

Cheraphilus echinulatus

Cheraphilus nanus

Cheraphilus trispinosus

Clibanarius misanthropus

Crangon echinulatus

Crangon fasciatus

Crangon nanus

Crangon norvegicus

Crangon spinosus
Systellaspis debilis (M. Edwards, 1881)

Acanthephyra purpurea M.

Edwards, 1881 or Acanthephyra

pelagica (Risso, 1816)

Philocheras fasciatus (Risso, 1816)

Alpheus glaber (Olivi, 1792)

Gennadas elegans (S.I. Smith, 1882)

Anapagurus laevis (Bell, 1846)

or Anapagurus breviaculeatus

Fenizia, 1937

Aristeomorpha foliacea (Risso, 1827) or Funchalia woodardii Johnson, 1867

Aristeus antennatus (Risso, 1816) or Funchalia woodardii Johnson, 1867

Homarus gammarus (Linnaeus, 1758)

Nephrops norvegicus (Linnaeus, 1758)

Atelecyclus rotundatus (Olivi, 1792)

Bathynectes longipes (Risso, 1816)

Calcinus tubularis (Linnaeus, 1767)

Callianassa tyrrhena (Petagna, 1792)

Callianassa subterranea (Montagu, 1808)

or Callianassa tyrrhena (Petagna, 1792)

Jaxea nocturna Nardo, 1847

Calocaris macandreae Bell, 1846 or

Callianassa subterranea (Montagu, 1808)

Cancer pagurus Linnaeus, 1758

Necora puber (Linnaeus, 1767)

Carcinus aestuarii Czerniavsky, 1884

Cestopagurus timidus (Roux, 1830) or

Nematopagurus longicornis M. Edwards and Bouvier, 1892

Philocheras echinulatus (Sars, 1861)

Philocheras bispinosus bispinosus

(Hailstone, 1835)

Philocheras trispinosus (Hailstone, 1835)

Clibanarius erythropus (Latreille, 1818)

Philocheras echinulatus (Sars, 1861)

Philocheras fasciatus (Risso, 1816)

Philocheras bispinosus bispinosus

(Hailstone, 1835)

Pontophilus norvegicus (Sars, 1861)

Pontophilus spinosus (Leach, 1815)

rator, Leach; Hyas araneus, (L.); Eupagurus bernhardus, L.; Galathea dispersa, Spence Bate; Crangon trispinosus (Hailstone); Cancer pagurus, L. Fish. Scot. Sci. Invest., 1909: 1-20. Williamson, H.C. - 1915. VI. Crustacea Decapoda. Larven. Nord. Plank. 18: 315-588.

Wilson, K.A. and Gore, R.H. - 1980. Studies on Decapod Crustacea from the Indian River region of Florida. XVII. Larval stages of Plagusia depressa (Fabricius, 1775) cultured under laboratory conditions (Brachyura, Grapsidae). Bull. Mar. Sci., 30(4): 776-789.

Wimpenny, R.S. and Titterington, E. -1963 . The tow-net plankton of lake Qarun, Egypt. 2. The stages of Leander squilla elegans Rathke. Notes Mem. Fish. Res., 14: 21-36.

Yagi, H. - 1986. Le developpement larvaire de la crevette rose Palaemon serratus (Pennant) (Palaemonidae, Decapoda, Crustacea). Mesogeé, 46(1): 29-43.

Yang, W.T. - 1968. The zoeae, megalopa, and the first crab of Epialtus dilatatus (Brachyura, Majidae) reared in the laboratory. Crustaceana, (Supl. 2): 181-202.

Yang, W.T. - 1971. The larval and postlarval development of Parthenope serrata reared in the laboratory and the systematic position of the Parthenopinae (Crustacea, Brachyura). Biol. Bull., 140: 166-189.

Scient. ed.: P. Abelló

Crangon trispinosus

Crangon vulgaris

Dorippe lanata

Dromia personatus

Dromia vulgaris

Dromidia larraburei

Eriphia spinifrons

Ethusa mascerone

Eupagurus bernhardus

Eupagurus excavatus

Eupagurus prideauxii

Euphema armata

Galathea nexa

Galathea rugosa

Galathodes tridentata

Gebia littoralis

Geryon tridens

Glaucothoe grimaldii

Glaucothoe rostrata

Gonoplax angulata

Gonoplax rhomboides

Hippolyte cranchii

Hippolyte prideauxiana

Homarus vulgaris

Homola cuvier

Inachus dorynchus

Inachus rostratus

Inachus scorpio

Inachus tetraodon

Lambrus angulifrons Parthenope angulifrons Latreille, 1825

Lambrus massena

Leander adspersus

Leander fabricii

Leander longirostris

Leander serratus

Leander squilla

Leander squilla elegans

Leander squilla typica

Leander xiphias

Leandri rectirostris

Liocarcinus puber

Macropipus corrugatus

Macropipus holsatus

Macropipus marmoreus
Philocheras trispinosus (Hailstone, 1835)

Crangon crangon (Linnaeus, 1758)

Medorippe lanata (Linnaeus, 1767)

Dromia personata (Linnaeus, 1758)

Dromia personata (Linnaeus, 1758)

Dromia personata (Linnaeus, 1758)

Eriphia verrucosa (Forskål, 1775)

Ethusa mascarone (Herbst, 1785)

Pagurus bernhardus (Linnaeus, 1758)

Pagurus alatus (Fabricius, 1775)

Pagurus prideauxi Leach, 1815

Gennadas elegans (S.I. Smith, 1882)

Galathea nexa Embleton, 1834 or

Galathea dispersa Bate, 1859

Munida rugosa (Fabricius, 1775)

Munidopsis serricornis (Lovén, 1853)

Upogebia deltaura (Leach, 1815) or

Upogebia pusilla (Petagna, 1792)

Geryon trispinosus (Herbst, 1803)

Calcinus tubularis (Linnaeus, 1767)

Dardanus calidus (Risso, 1826)

Goneplax rhomboides (Linnaeus, 1758)

Goneplax rhomboides (Linnaeus, 1758)

Thoralus cranchii (Leach,1817)

Hippolyte inermis Leach, 1815

Homarus gammarus (Linnaeus, 1758)

Homola barbata (Fabricius, 1793)

Oplophorus spinosus (Brullé, 1839)

Inachus phalangium (Fabricius, 1775)

Macropodia rostrata (Linnaeus, 1761)

Inachus dorsettensis (Pennant, 1777) or

Inachus thoracicus (Roux, 1830)

Pisa armata (Latreille, 1803)

Parthenope massena (Roux, 1830) Palaemon adspersus Rathke, 1837 Palaemon adspersus Rathke, 1837 Palaemon longirostris M. Edwards, 1837 Palaemon serratus (Pennant, 1777) Palaemon elegans Rathke, 1837 Palaemon elegans Rathke, 1837 Palaemon elegans Rathke, 1837 Palaemon xiphias Riso, 1816 Palaemon adspersus Rathke, 1837 Necora puber (Linnaeus, 1767)

Liocarcinus corrugatus (Pennant, 1777)

Liocarcinus holsatus (Fabricius, 1798)

Liocarcinus marmoreus (Leach, 1814) 
Macropipus puber

Macropipus pusillus

Macropodia aegyptia

Macropodia longirostris

Macropodia phalangium

Macropodia rostratus

Maia squinado

Maja verrucosa

Megalopa armata

Megalopa montagui

Miersia clavigera

Monoculus taurus

Munida banffica

Munida rugosa

Munidopsis tridentata

Nematocarcinus ensifer

Nematocarcinus ensifer exilis

Pagure misanthrope

Paguristes oculatus

Pagurus alatus

Pagurus arrosor

Pagurus chiroacanthus

Pagurus misanthropus

Pagurus prideaux

Pagurus sculptimanus

Pagurus variabilis

Palaemon fabricii

Palaemon squilla

Palaemon variabilis

Palaemon varians

Palinurus vulgaris

Pandalus (Dichelopandalus)

bonnieri

Pandalus (Pandalina) brevirostris

Pandalus brevirostris

Parapandalus richardi

Pasiphaë sulcatifrons

Pasiphaea principalis

Pasiphaea tarda

Passiphaea tarda

Penaeus japonicus

Penaeus kerathurus

Penaeus membranaceus

Penaeus siphonocerus

Penaeus trisulcatus

Percnon gibbesii

Perimela (Pirimela) denticulata

Phyllosoma mediterranea

Pinnotheres Marioni

Pinnotheres pinnotheres

Pinnotheres veterum

Pirimela denticulata

Pisa biaculeata

Pisa gibbsi

Pisidia longimana
Necora puber (Linnaeus, 1767)

Liocarcinus pusillus (Leach, 1815)

Macropodia deflexa Forest, 1978

Macropodia tenuirostris (Leach, 1814)

Macropodia rostrata (Linnaeus, 1761)

Macropodia rostrata (Linnaeus, 1761)

Maja squinado (Herbst, 1788)

Maja crispata (Risso, 1827)

Cancer pagurus Linnaeus, 1758

Liocarcinus depurator (Linnaeus, 1758)

Lysmata seticaudata (Risso, 1816)

Carcinus maenas (Linnaeus, 1758)

Munida rugosa (Fabricius, 1775)

Munida rugosa (Fabricius, 1775) or

Munida sarsi Huus, 1935

Munidopsis serricornis (Lovén, 1853)

Nematocarcinus exilis Bate, 1888

Nematocarcinus exilis Bate, 1888

Clibanarius erythropus (Latreille, 1818)

Paguristes eremita (Linnaeus, 1767)

Pagurus alatus (Fabricius, 1775) or

Pagurus excavatus (Herbst, 1791)

Dardanus arrosor (Herbst, 1796)

Anapagurus chiroacanthus (Lilljeborg,

1856)

Clibanarius erythropus (Latreille, 1818)

Pagurus prideauxi Leach, 1815

Pagurus forbesii Bell, 1845

Pagurus alatus (Fabricius, 1775)

Palaemon adspersus Rathke, 1837

Palaemon elegans Rathke, 1837

Palaemonetes varians (Leach, 1814

Palaemonetes varians (Leach, 1814)

Palinurus elephas (Fabricius, 1787)

Dichelopandalus bonnieri (Caullery, 1896)

Pandalina brevirostris (Rathke, 1843)

Pandalina brevirostris (Rathke, 1843)

Stylopandalus richardi (Coutière, 1905)

Parapasiphae sulcatifrons S. I. Smith, 1884

Pasiphaea multidentata Esmark, 1866

Pasiphaea tarda Kröyer, 1865 or

Pasiphaea sivado (Risso, 1816)

Pasiphaea multidentata Esmark, 1866

Marsupenaeus japonicus (Bate, 1888)

Melicertus kerathurus (Forskål, 1775)

Solenocera membranacea (Risso, 1816)

Solenocera membranacea (Risso, 1816)

Melicertus kerathurus (Forskål, 1775)

Percnon gibbesi (H.H. Edwards, 1853)

Pirimela denticulata (Montagu, 1808)

Scyllarus arctus (Linnaeus, 1758)

Neopinnotheres pinnotheres (Linnaeus, 1758)

Neopinnotheres pinnotheres (Linnaeus, 1758)

Neopinnotheres pinnotheres (Linnaeus,

1758) or Pisa tetraodon (Pennant, 1777)

Pirimela denticulata (Montagu, 1808)

or Portumnus latipes (Pennant, 1777)

Pisa armata (Latreille, 1803)

Pisa armata (Latreille, 1803)

Pisidia longicornis (Linnaeus, 1767)
Pluteocaris sp.

Polybius henslowi

Pontocaris cataphracta

Pontocaris cataphractus

Pontocaris lacazei

Pontonia custos

Pontonia phallusiae

Pontonia tyrrehena

Porcellana (Polyonyx)

macrocheles

Porcellana bluteli

Porcellana longicornis

Porcellana platycheles

Portumnus biguttatus

Portunus arcuatus

Portunus corrugatus

Portunus depurator

Portunus holsatus

Portunus maenas

Portunus marmoreus

Portunus puber

Portunus pusillus

Processa canaliculata

Processa edulis crassipes

Processa edulis edulis

Processa modica carolii

Processa modica modica

Processa nouveli holthuisi

Processa nouveli nouveli

Pseudibacus veranyi

Sergestes arcticus

Sergestes corniculum

Sergestes cornutus

Sergestes diaponticus

Sergestes nudus

Sergestes robustus

Sicyonia sculpta

Solenocera siphonocera

Spirontocaris cranchi

Spirontocaris occulta

Spiropagurus chiroacanthus

Stenorhynchus phalangium

Synalpheus laevimanus

Taxea nocturna

Thenus orientalis

Thia polita

Thia residua

Trachelifer

Tritodynamia atlantica

Upogebia littoralis

Xantho hydrophilus

Xantho rivulosus

Zoea Virbii
Percnon gibbesi (H.M. Edwards, 1853)

Polybius henslowii Leach, 1820

Aegaeon cataphractus (Olivi, 1792 )

Aegaeon cataphractus (Olivi, 1792)

Aegaeon lacazei (Gourret, 1887)

Pontonia pinnophylax (Otto, 1821)

Pontonia flavomaculata Heller,1864

Pontonia pinnophylax (Otto, 1821)

Porcellana platycheles (Pennant, 1777) Pisidia bluteli (Risso, 1816)

Pisidia longicornis (Linnaeus, 1767)

Porcellana platycheles (Pennant, 1777)

or Pisidia longicornis (Linnaeus, 1767)

Xaiva biguttata (Risso,1816)

Liocarcinus arcuatus (Leach, 1814)

Liocarcinus corrugatus (Pennant, 1777)

Liocarcinus depurator (Linnaeus, 1758)

Liocarcinus holsatus (Fabricius, 1798)

Carcinus maenas (Linnaeus, 1758)

Liocarcinus marmoreus (Leach, 1814)

Necora puber (Linnaeus, 1767)

Liocarcinus pusillus (Leach,1815)

Processa edulis (Risso, 1816) or Processa

nouveli Al-Adhub and Williamson, 1975

Processa edulis (Risso, 1816)

Processa edulis (Risso, 1816)

Processa modica Williamson, 1979

Processa modica Williamson, 1979

Processa nouveli Al-Adhub and

Williamson, 1975

Processa nouveli Al-Adhub and

Williamson, 1975

Scyllarides latus (Latreille, 1803)

Sergestes arcticus Kroyer, 1855 or Sergia robusta (S.I. Smith, 1882)

Sergestes curvatus Crosnier and Forest,

1973 or Sergestes henseni (Ortmann, 1893)

Sergestes cornutus Kröyer, 1855 or

Sergestes atlanticus H.M. Edwards, 1830

Sergestes armatus Kröyer, 1855 or

Sergestes vigilax Stimpson, 1860

Sergestes sargassi Ortmann,1893

Sergia japonica Bate, 1881or Sergia

robusta (S.I. Smith, 1882)

Sicyonia carinata (Brünnich, 1768)

Solenocera membranacea (Risso, 1816)

Thoralus cranchii (Leach, 1817)

Eualus occultus (Lebour, 1936)

Anapagurus chiroacanthus (Lilljeborg, 1856)

Macropodia rostrata (Linnaeus, 1761)

Synalpheus gambarelloides (Nardo, 1847)

Jaxea nocturna Nardo, 1847

Scyllarides latus (Latreille, 1803)

Thia scutellata (Fabricius, 1793)

Thia scutellata (Fabricius, 1793)

Jaxea nocturna Nardo, 1847

Asthenognathus atlanticus Monod, 1933

Upogebia pusilla (Petagna, 1792)

Xantho pilipes M. Edwards, 1867 or

Xantho poressa (Olivi, 1792)

Xantho poressa (Olivi, 1792)

Clibanarius erythropus (Latreille, 1818) 